\title{
SAMPLE PATH LARGE DEVIATIONS FOR LÉVY PROCESSES AND RANDOM WALKS WITH WEIBULL INCREMENTS
}

\author{
By Mihail Bazhba* ${ }^{*}$, Jose Blanchet ${ }^{\dagger}$, Chang-Han Rhee $^{\ddagger}$ And Bert Zwart* \\ Centrum Wiskunde 83 Informatica ${ }^{*}$, Stanford University ${ }^{\dagger}$, and Northwestern University ${ }^{\ddagger}$
}

\begin{abstract}
We study sample path large deviations for Lévy processes and random walks with heavy-tailed jump-size distributions that are of Weibull type. Our main results include an extended form of an LDP (large deviations principle) in the $J_{1}$ topology, and a full LDP in the $M_{1}^{\prime}$ topology. The rate function can be represented as the solution to a quasi-variational problem. The sharpness and applicability of these results are illustrated by a counterexample proving the nonexistence of a full LDP in the $J_{1}$ topology, and by an application to a first passage problem.
\end{abstract}

1. Introduction. In this paper, we develop sample path large deviations for Lévy processes and random walks, assuming the jump sizes have a semi-exponential distribution. Specifically, let $X(t), t \geq 0$, be a centered Lévy process with positive jumps and Lévy measure $\nu$ which has nonnegative support. Assume that $-\log \nu[x, \infty)$ is regularly varying of index $\alpha \in(0,1)$ and define $\bar{X}_{n}=\left\{\bar{X}_{n}(t), t \in[0,1]\right\}$, with $\bar{X}_{n}(t)=X(n t) / n, t \geq 0$. We are interested in large deviations of $\bar{X}_{n}$.

The investigation of tail estimates of the one-dimensional distributions of $\bar{X}_{n}$ (or random walks with heavy-tailed step size distribution) was initiated in Nagaev $(1969,1977)$. The state of the art of such results is well summarized in Borovkov and Borovkov (2008); Denisov, Dieker and Shneer (2008); Embrechts, Klüppelberg and Mikosch (1997); Foss, Korshunov and Zachary (2011). In particular, Denisov, Dieker and Shneer (2008) describe in detail how fast $x$ needs to grow with $n$ for the asymptotic relation

$$
\mathbf{P}(X(n)>x)=n \mathbf{P}(X(1)>x)(1+o(1))
$$

to hold, as $n \rightarrow \infty$. If (1.1) is valid, the so-called principle of one big jump is said to hold. It turns out that, if $x$ increases linearly with $n$, this principle holds if $\alpha<1 / 2$ and does not hold if $\alpha>1 / 2$, and the asymptotic behavior of $\mathbf{P}(X(n)>x)$ becomes more complicated. When studying more general functionals of $X$ it becomes natural to consider logarithmic asymptotics, as is common in large deviations theory, cf. Dembo and Zeitouni (2009); Gantert (1998); Gantert, Ramanan and Rembart (2014).

The study of large deviations of sample paths of processes with Weibullian increments is relatively limited. The only paper we are aware of is Gantert (1998), where the inverse contraction principle is applied to obtain a large deviation principle in the $L_{1}$ topology. As noted in

MSC 2010 subject classifications: Primary 60F10; secondary 60G17

Keywords and phrases: sample path large deviations, Lévy processes, random walks, heavy tails 
Duffy and Sapozhnikov (2008) this topology is not suitable for many applications -ideally one would like to work with the $J_{1}$ topology, see equation (2.2).

Let us now describe precisely our results. We first develop an extended LDP (large deviations principle) in the $J_{1}$ topology, i.e. there exists a rate function $I(\cdot)$ such that

$$
\liminf _{n \rightarrow \infty} \frac{\log \mathbf{P}\left(\bar{X}_{n} \in A\right)}{L(n) n^{\alpha}} \geq-\inf _{x \in A} I(x) .
$$

if $A$ is open, and

$$
\limsup _{n \rightarrow \infty} \frac{\log \mathbf{P}\left(\bar{X}_{n} \in A\right)}{L(n) n^{\alpha}} \leq-\lim _{\epsilon \downarrow 0} \inf _{x \in A^{\epsilon}} I(x) .
$$

if $A$ is closed. Here $A^{\epsilon}=\{x: d(x, A) \leq \epsilon\}$. The rate function $I$ is given by

$$
I(\xi)= \begin{cases}\sum_{t: \xi(t) \neq \xi\left(t^{-}\right)}(\xi(t)-\xi(t-))^{\alpha} & \text { if } \xi \in \mathbb{D}_{\leqslant \infty}[0,1], \\ \infty & \text { otherwise }\end{cases}
$$

where $\mathbb{D}_{\leqslant \infty}[0,1]$ is the subspace of $\mathbb{D}[0,1]$ consisting of non-decreasing pure jump functions vanishing at the origin and continuous at 1 . (As usual, $\mathbb{D}[0,1]$ is the space of cadlag functions from $[0,1]$ to $\mathbb{R}$.)

The notion of an extended large deviations principle has been introduced by Borovkov and Mogulskii (2010). We derive this result as follows: we use a suitable representation for the Lévy process in terms of Poisson random measures, allowing us to decompose the process into the contribution generated by the $k$ largest jumps, and the remainder. The contribution generated by the $k$ largest jumps is a step function for which we obtain the large deviations behavior by Bryc's inverse Varadhan lemma (see e.g. Theorem 4.4.13 of Dembo and Zeitouni, 2010). The remainder term is tamed by modifying a concentration bound due to Jelenković and Momčilović (2003).

To combine both estimates we need to consider the $\epsilon$-fattening $A^{\epsilon}$ of the set $A$, which precludes us from obtaining a full LDP. To show that our approach cannot be improved, we construct a set $A$ that is closed in the Skorokhod $J_{1}$ topology for which the large deviation upper bound does not hold. In this sense, our extended large deviations principle can be seen as optimal. This is in line with the observation made for the regularly varying Lévy processes and random walks (Rhee, Blanchet and Zwart, 2016), for which the full LDP w.r.t. $J_{1}$ topology in a classical sense is shown to be unobtainable as well.

Following a similar proof strategy, we also derive an extended sample path LDP for random walks in $\mathbb{D}[0,1]$. There are however also some differences. The distributional representation of our random walk is different from the continuous-time case. More importantly, the resulting rate function differs at time 1 , since the rescaled random walk always has a jump at time 1 . We present an exact formulation of this result in Theorem 2.2 .

We derive several implications of our extended LDP that facilitate its use in applications. First of all, if a Lipschitz functional $\phi$ of $\bar{X}_{n}$ is chosen for which the function $I_{\phi}(y)=\inf _{x: \phi(x)=y} I(x)$ is a good rate function, then $\phi\left(X_{n}\right)$ satisfies an LDP. We illustrate this procedure by considering an example concerning the probability of ruin for an insurance company where large claims are reinsured. 
A second implication is a sample path LDP in the $M_{1}^{\prime}$ topology. We show that the rate function $I$ is good in this topology, allowing us to conclude $\lim _{\epsilon \downarrow 0} \inf _{x \in A^{\epsilon}} I(x)=\inf _{x \in A} I(x)$, if $A$ is closed in the $M_{1}^{\prime}$ topology. An overview of the $M_{1}^{\prime}$ topology can be seen in Appendix A or in Puhalskii and Whitt (1997). The LDP for the $M_{1}^{\prime}$ topology in this paper is applied in Bazhba et al. (2019) to obtain tail asymptotics of the queue length in the multiple server queue with heavy tailed Weibull service times. In that work, we show that the most likely number of big jobs that cause a large queue length is determined by the solution of an $L^{\alpha}$-norm minimization problem for $\alpha \in(0,1)$. This result essentially answers a question posed by Sergey Foss at the Erlang Centennial conference in 2009. For earlier conjectures on this problem we refer to Whitt (2000).

We note that both implications of our extended LDP constitute two complementary tools, and that the two aforementioned applications are dealt with using precisely one of these tools. In particular, the functional in the reinsurance example is not continuous in the $M_{1}^{\prime}$ topology, and the most likely paths in the queueing application are discontinuous at time 0 , rendering the $J_{1}$ or $M_{1}$ topologies useless.

Another application of our results, which will be pursued in detail elsewhere, arises in the large deviations analysis of Markov random walks. More precisely, when studying $\bar{X}_{n}(t)=\sum_{k=1}^{\lfloor n t\rfloor} f\left(Y_{k}\right) / n$, where $Y_{k}, k \geq 0$ is a geometrically ergodic Markov chain and $f(\cdot)$ is a given measurable function. Classical large deviations results pioneered by Donsker and Varadhan on this topic (see, for example, Donsker and Varadhan, 1976) and the more recent treatment in Kontoyiannis and Meyn (2005), impose certain Lyapunov-type assumptions involving the underlying function, $f(\cdot)$.

These assumptions are not merely technical requirements, but are needed for a large deviations theory with a linear (in $n$ ) speed function (as opposed to sublinear as we obtain here). Even in simple cases (e.g. Blanchet, Glynn and Meyn, 2011; Duffy and Meyn, 2014) the case of unbounded $f(\cdot)$ can result in a sublinear large deviations scaling of the type considered here. For Harris chains, this can be seen by splitting $\bar{X}_{n}(\cdot)$ into cycles. Each term corresponding to a cycle can be seen as the area under a curve generated by $f(Y$.). For linear $f$, this results in a contribution towards $\bar{X}_{n}(\cdot)$ which often is roughly proportional to the square of the cycle. Hence, the behavior of $\bar{X}_{n}(1)$ is close to that of a sum of i.i.d. Weibull-type random variables. To summarize, the main results of this paper can be applied to significantly extend the classical Donsker-Varadhan theory to unbounded functionals of Markov chains.

This paper is organized as follows. Section 2 introduces notation and presents extended LDP's. These are complemented in Section 3 by LDP's of Lipschitz functionals, LDP's in the $M_{1}^{\prime}$ topology, and a counterexample. Section 4 is considering an application to boundary crossing probabilities with moderate jumps. Additional proofs are presented in Section 5. The appendix develops further details about the $M_{1}^{\prime}$ topology that are needed in the body of the paper.

2. Sample path LDPs. In this section, we discuss sample path large deviations for Lévy processes and random walks. Before presenting the main results, we start with general background. Let $(\mathbb{S}, d)$ be a metric space, and $\mathcal{T}$ denote the topology induced by the metric $d$. Let $X_{n}$ be a sequence of $\mathbb{S}$-valued random variables. Let $A^{\epsilon} \triangleq\{\xi \in \mathbb{S}: d(\xi, A) \leq \epsilon\}$ where $d(\xi, A)=$ $\inf _{\zeta \in A} d(\xi, \zeta)$, and $A^{-\epsilon} \triangleq\{\xi \in \mathbb{S}: d(\xi, \zeta) \leq \epsilon$ implies $\zeta \in A\}$. Let $I$ be a non-negative lower semicontinuous function on $\mathbb{S}$, and $a_{n}$ be a sequence of positive real numbers that tends to infinity as 
$n \rightarrow \infty$. We say that $X_{n}$ satisfies the LDP in $(\mathbb{S}, \mathcal{T})$ with speed $a_{n}$ and the rate function $I$ if

$$
-\inf _{x \in A^{\circ}} I(x) \leq \liminf _{n \rightarrow \infty} \frac{\log \mathbf{P}\left(X_{n} \in A\right)}{a_{n}} \leq \limsup _{n \rightarrow \infty} \frac{\log \mathbf{P}\left(X_{n} \in A\right)}{a_{n}} \leq-\inf _{x \in A^{-}} I(x)
$$

for any measurable set $A$. Here, $A^{\circ}$ and $A^{-}$are respectively the interior and the closure of the set $A$. We say that $X_{n}$ satisfies the extended LDP in $(\mathbb{S}, \mathcal{T})$ with speed $a_{n}$ and the rate function $I$ if

$$
-\inf _{x \in A^{\circ}} I(x) \leq \liminf _{n \rightarrow \infty} \frac{\log \mathbf{P}\left(X_{n} \in A\right)}{a_{n}} \leq \limsup _{n \rightarrow \infty} \frac{\log \mathbf{P}\left(X_{n} \in A\right)}{a_{n}} \leq-\lim _{\epsilon \rightarrow 0} \inf _{x \in A^{\epsilon}} I(x)
$$

for any measurable set $A$. The next proposition provides the key framework for proving our main results.

Proposition 2.1. Let $I$ and $I_{k}, k \geq 1$ be rate functions. Suppose that for each $n, X_{n}$ has a sequence of approximations $\left\{Y_{n}^{k}\right\}_{k=1, \ldots}$ such that

(i) For each $k, Y_{n}^{k}$ satisfies the extended $L D P$ in $(\mathbb{S}, \mathcal{T})$ with speed $a_{n}$ and the rate function $I_{k}$;

(ii) For each closed set F,

$$
\lim _{k \rightarrow \infty} \inf _{x \in F} I_{k}(x) \geq \inf _{x \in F} I(x)
$$

(iii) For each $\delta>0$ and each open set $G$, there exist $\epsilon>0$ and $K \geq 0$ such that $k \geq K$ implies

$$
\inf _{x \in G^{-\epsilon}} I_{k}(x) \leq \inf _{x \in G} I(x)+\delta
$$

(iv) For every $\epsilon>0$ it holds that

$$
\limsup _{k \rightarrow \infty} \limsup _{n \rightarrow \infty} \frac{1}{a_{n}} \log \mathbf{P}\left(d\left(X_{n}, Y_{n}^{k}\right)>\epsilon\right)=-\infty
$$

Then, $X_{n}$ satisfies the extended $L D P$ in $(\mathbb{S}, \mathcal{T})$ with speed $a_{n}$ and the rate function $I$.

The proof of this proposition is provided in Section 5. We conclude this section with an immediate implication of Proposition 2.1.

Corollary 2.1. Suppose that $Y_{n}$ satisfies the extended LDP in $(\mathbb{S}, \mathcal{T})$ with speed $a_{n}$ and the rate function $I$. If for each $\epsilon>0$,

$$
\limsup _{n \rightarrow \infty} \frac{1}{a_{n}} \log \mathbf{P}\left(d\left(X_{n}, Y_{n}\right)>\epsilon\right)=-\infty
$$

then $X_{n}$ satisfies the extended $L D P$ in $(\mathbb{S}, \mathcal{T})$ with speed $a_{n}$ and the rate function $I$.

Proof. Let $Y_{n}^{k} \triangleq Y_{n}$ and $I_{k} \triangleq I$ for $k=1,2, \ldots$ Then, (i) and (ii) of Proposition 2.1 are trivially satisfied. For (iii), we note that by the definition of $G^{-\epsilon}$, for each $\delta>0$ and $G$ an open set, there exists $\epsilon>0$ such that

$$
\inf _{x \in G^{-\epsilon}} I(x) \leq \inf _{x \in G} I(x)+\delta,
$$

and hence, (iii) is satisfied for $I_{k}=I$. Since (iv) is also satisfied by the assumption, all the conditions of Proposition 2.1 is satisfied and the conclusion of the corollary follows. 
2.1. Extended sample path LDP for Lévy processes. Throughout the rest of this paper we assume that

A1. $X$ is a real-valued Lévy process with Lévy measure $\nu$ which has non-negative support satisfying $\nu[x, \infty)=\exp \left(-L(x) x^{\alpha}\right)$ where $\alpha \in(0,1)$ and $L(\cdot)$ is slowly varying at infinity.

A2. The mapping $x \mapsto L(x) x^{\alpha-1}$ is non-increasing for sufficiently large $x$.

Let $\bar{X}_{n}(t)$ denote the centered and scaled process:

$$
\bar{X}_{n}(t) \triangleq \frac{1}{n} X(n t)-t \mathbf{E} X(1) .
$$

Let $\mathbb{D}[0,1]$ denote the Skorokhod space - space of càdlàg functions from $[0,1]$ to $\mathbb{R}$ - and $\mathcal{T}_{J_{1}}$ denote the $J_{1}$ Skorokhod topology on $\mathbb{D}[0,1]$. That is, $\mathbb{D}[0,1]$ is metrized by the usual Skorokhod metric

$$
d_{J_{1}}(\xi, \zeta) \triangleq \inf _{\lambda \in \Lambda}\left\{\max \left\{\|\xi(t)-\zeta(\lambda(t))\|_{\infty},\|\lambda(t)-e(t)\|_{\infty}\right\}\right\}
$$

where $\xi, \zeta \in \mathbb{D}[0,1], \lambda$ is a non-decreasing homeomorphism of $[0,1]$ onto itself, $\Lambda$ is the set of such homeomorphisms, $e(t)=t$ is the identity map, and $\|\xi\|_{\infty}=\sup _{t \in[0,1]}|\xi(t)|$ is the supremum norm. We say that $\xi \in \mathbb{D}[0,1]$ is a pure jump function if $\xi=\sum_{i=1}^{\infty} x_{i} \mathbb{1}_{\left[u_{i}, 1\right]}$ for some $x_{i}$ 's and $u_{i}$ 's such that $x_{i} \in \mathbb{R}$ and $u_{i} \in(0,1)$ for each $i$ and $u_{i}$ 's are all distinct. Let $\mathbb{D}_{\leqslant \infty}[0,1]$ denote the subspace of $\mathbb{D}[0,1]$ consisting of non-decreasing pure jump functions vanishing at the origin and continuous at 1. For the rest of the paper, if there is no confusion regarding the domain of a function space, we will omit the domain and simply write, for example, $\mathbb{D}_{\leqslant \infty}$ instead of $\mathbb{D}_{\leqslant \infty}[0,1]$. The next theorem is the main result of this paper.

TheOREM 2.1. $\quad \bar{X}_{n}$ satisfies the extended large deviation principle in $\left(\mathbb{D}, \mathcal{T}_{J_{1}}\right)$ with speed $L(n) n^{\alpha}$ and rate function

$$
I(\xi)= \begin{cases}\sum_{t: \xi(t) \neq \xi(t-)}(\xi(t)-\xi(t-))^{\alpha} & \text { if } \xi \in \mathbb{D}_{\leqslant \infty}, \\ \infty, & \text { otherwise. }\end{cases}
$$

That is, for any measurable A,

$$
-\inf _{\xi \in A^{\circ}} I(\xi) \leq \liminf _{n \rightarrow \infty} \frac{\log \mathbf{P}\left(\bar{X}_{n} \in A\right)}{L(n) n^{\alpha}} \leq \limsup _{n \rightarrow \infty} \frac{\log \mathbf{P}\left(\bar{X}_{n} \in A\right)}{L(n) n^{\alpha}} \leq-\lim _{\epsilon \rightarrow 0} \inf _{\xi \in A^{\epsilon}} I(\xi),
$$

where $A^{\epsilon} \triangleq\left\{\xi \in \mathbb{D}: d_{J_{1}}(\xi, \zeta) \leq \epsilon\right.$ for some $\left.\zeta \in A\right\}$.

REMARK 2.1. Note that it is straightforward to extend Theorem 2.1 to spectrally two-sided Lévy processes. For instance, suppose that the Lévy measure $\nu$ of the process $X$ has Weibull tail $\nu[x, \infty)=\exp \left(-L(x) x^{\alpha}\right)$ where $\alpha \in(0,1), L(x) x^{\alpha-1}$ satisfies assumption $A 2$, and $\nu(-\infty, x]$ is light-tailed. We can consider a decomposition of $\bar{X}_{n}$ into a spectrally positive part $\bar{Y}_{n}$ and a spectrally negative part $\bar{X}_{n}-\bar{Y}_{n}$. Then, $\bar{Y}_{n}$ satisfies the LDP in Theorem 2.1. On the other hand, since $\bar{X}_{n}-\bar{Y}_{n}$ is light-tailed, it satisfies a sample path LDP with linear speed-see Mogulskii (1993). This implies that the condition Corollary 2.1 is satisfied, which, allows one to apply Corollary 2.1 with $Y_{n}$, and we conclude that $\bar{X}_{n}$ satisfies the same LDP as the one in Theorem 2.1. 
Recall that $X_{n}(\cdot) \triangleq X(n \cdot)$ has Itô representation

$$
X_{n}(s)=n s a+B(n s)+\int_{x<1} x[\hat{N}([0, n s] \times d x)-n s \nu(d x)]+\int_{x \geq 1} x \hat{N}([0, n s] \times d x),
$$

with $a$ a drift parameter, $B$ a Brownian motion, and $\hat{N}$ a Poisson random measure with mean measure Leb $\times \nu$ on $[0, n] \times(0, \infty)$; Leb here denotes the Lebesgue measure. All terms in (2.5) are independent. We will see that the large deviation behavior is dominated by the last term of (2.5). It turns out to be convenient to consider the following distributional representation of the centered and scaled version of the last term:

$$
\bar{Y}_{n}(\cdot) \triangleq \frac{1}{n} \sum_{l=1}^{N(n \cdot)}\left(Z_{l}-\mathbf{E} Z\right) \stackrel{\mathcal{D}}{=} \frac{1}{n} \int_{x \geq 1} x \hat{N}([0, n \cdot] \times d x)-\frac{1}{n}(\mathbf{E} Z) \hat{N}([0, n \cdot] \times[1, \infty))
$$

where $N(t) \triangleq \hat{N}([0, t] \times[1, \infty))$ is a Poisson process with arrival rate $\nu_{1} \triangleq \nu[1, \infty)$, and $Z_{i}$ 's are i.i.d. copies of $Z$ such that $\mathbf{P}(Z \geq x)=\nu[x \vee 1, \infty) / \nu_{1}$, independent of $N$. To facilitate the proof of Theorem 2.1, we consider a further decomposition of $\bar{Y}_{n}$ into two pieces, one of which consists of the big increments, and the other one keeps the residual fluctuations. To be more specific, we introduce an extra notation for the rank of the increments. Given $N(n)$, define $\mathbf{S}_{N(n)}$ to be the set of all permutations of $\{1, \ldots, N(n)\}$. Let $R_{n}:\{1, \ldots, N(n)\} \rightarrow\{1, \ldots, N(n)\}$ be a random permutation of $\{1, \ldots, N(n)\}$ sampled uniformly from $\Sigma_{n} \triangleq\left\{\sigma \in \mathbf{S}_{N(n)}: Z_{\sigma^{-1}(1)} \geq \cdots \geq Z_{\sigma^{-1}(N(n))}\right\}$. In words, $R_{n}(i)$ is the rank of $Z_{i}$ among $\left\{Z_{1}, \ldots, Z_{N(n)}\right\}$ when sorted in decreasing order with the ties broken uniformly randomly. Now, we see that

$$
\bar{Y}_{n}(t)=\underbrace{\frac{1}{n} \sum_{i=1}^{N(n t)} Z_{i} \mathbb{1}_{\left\{R_{n}(i) \leq k\right\}}}_{\triangleq \bar{J}_{n}^{k}(t)}+\underbrace{\frac{1}{n} \sum_{i=1}^{N(n t)}\left(Z_{i} \mathbb{1}_{\left\{R_{n}(i)>k\right\}}-\mathbf{E} Z\right)}_{\triangleq \bar{H}_{n}^{k}(t)} .
$$

The proof of Theorem 2.1 is straightforward once the following technical lemmas are in our hands; their proofs are provided in Section 5 . Let $\mathbb{D}_{\leqslant k}$ denote the subspace of $\mathbb{D}_{\leqslant \infty}$ consisting of paths that have less than or equal to $k$ discontinuities.

LEMma 2.1. For each fixed $k, \bar{J}_{n}^{k}$ satisfies the $L D P$ in $\left(\mathbb{D}, \mathcal{T}_{J_{1}}\right)$ with speed $L(n) n^{\alpha}$ and the rate function

$$
I_{k}(\xi)= \begin{cases}\sum_{t \in[0,1]}(\xi(t)-\xi(t-))^{\alpha} & \text { if } \xi \in \mathbb{D}_{\leqslant k} \\ \infty & \text { otherwise }\end{cases}
$$

Recall that $A^{-\epsilon} \triangleq\left\{\xi \in \mathbb{D}: d_{J_{1}}(\xi, \zeta) \leq \epsilon\right.$ implies $\left.\zeta \in A\right\}$.

Lemma 2.2. For each $\delta>0$ and each open set $G$, there exist $\epsilon>0$ and $K \geq 0$ such that for any $k \geq K$

$$
\inf _{\xi \in G^{-\epsilon}} I_{k}(\xi) \leq \inf _{\xi \in G} I(\xi)+\delta
$$


Let $B_{J_{1}}(\xi, \epsilon)$ be the open ball w.r.t. the $J_{1}$ Skorokhod metric centered at $\xi$ with radius $\epsilon$ and $B_{\epsilon} \triangleq B_{J_{1}}(0, \epsilon)$.

LEMma 2.3. For every $\epsilon>0$ it holds that

$$
\lim _{k \rightarrow \infty} \limsup _{n \rightarrow \infty} \frac{1}{L(n) n^{\alpha}} \log \mathbf{P}\left(\left\|\bar{H}_{n}^{k}\right\|_{\infty}>\epsilon\right)=-\infty .
$$

Proof of Theorem 2.1. For this proof, we use the following representation of $\bar{X}_{n}$ :

$$
\bar{X}_{n} \stackrel{\mathcal{D}}{=} \bar{Y}_{n}+\bar{R}_{n}=\bar{J}_{n}^{k}+\bar{H}_{n}^{k}+\bar{R}_{n},
$$

where $\bar{R}_{n}(s)=\frac{1}{n} B(n s)+\frac{1}{n} \int_{|x| \leq 1} x[N([0, n s] \times d x)-n s \nu(d x)]+\frac{1}{n}(\mathbf{E} Z) \hat{N}([0, n \cdot] \times[1, \infty))-\nu_{1} t$. Next, we verify the conditions of Proposition 2.1. Lemma 5.1 confirms that $I$ is lower semi-continuous. Lemma 2.1 verifies (i). To see that (ii) is satisfied, note that $I_{k}(\xi) \geq I(\xi)$ for any $\xi \in \mathbb{D}$. Lemma 2.2 verifies (iii). Since $d_{J_{1}}\left(\bar{X}_{n}, \bar{J}_{n}^{k}\right) \leq\left\|\bar{H}_{n}^{k}\right\|_{\infty}+\left\|\bar{R}_{n}\right\|_{\infty}$, Lemma 2.3 and $\lim \sup _{n \rightarrow \infty} \frac{1}{L(n) n^{\alpha}} \log \mathbf{P}\left(\left\|\bar{R}_{n}\right\|_{\infty}>\right.$ $\epsilon)=-\infty$ implies (iv). Note that $\bar{R}_{n}$ is a Lévy process whose moment generating function is finite everywhere, and hence, the LDP upper bound in Theorem 2.5 of Mogulskii (1993) applies (with linear speed) to $\mathbf{P}\left(d_{J_{1}}\left(0, \bar{R}_{n}\right)>\epsilon\right)$. This, in turn, implies that $\lim \sup _{n \rightarrow \infty} \frac{1}{L(n) n^{\alpha}} \log \mathbf{P}\left(\left\|\bar{R}_{n}\right\|_{\infty}>\right.$ $\epsilon)=-\infty$. Now, the conclusion of the theorem follows from Proposition 2.1.

2.2. Extended $L D P$ for random walks. Let $S_{n} \triangleq Z_{1}+\cdots+Z_{n}$ where $Z_{i}$ 's are non-negative random variables. Consider the centered and scaled process $\bar{S}_{n}=\left\{\bar{S}_{n}(t), t \in[0,1]\right\}$ where $\bar{S}_{n}(t) \triangleq$ $\frac{1}{n} \sum_{i=1}^{[n t]}\left(Z_{i}-\mathbf{E} Z\right), t \in[0,1]$. We assume that $\mathbf{P}(Z \geq x)=\exp \left(-L(x) x^{\alpha}\right)$ where $\alpha \in(0,1)$ and $L(\cdot)$ is a slowly varying function. We also assume A2 is in force as in Section 2.1. The goal of this section is to prove an extended LDP for $\bar{S}_{n}$. Let $\tilde{\mathbb{D}}_{\leqslant \infty}$ denote the subspace of $\mathbb{D}$ consisting of non-decreasing pure jump functions vanishing at the origin (not necessarily continuous at 1 , though). Let $\tilde{\mathbb{D}}_{\leqslant k}$ denote the subspace of $\tilde{\mathbb{D}}_{\leqslant \infty}$ consisting of paths that have less than or equal to $k$ discontinuities. Define $\tilde{I}$ as follows:

$$
\tilde{I}(\xi)= \begin{cases}\sum_{t: \xi(t) \neq \xi(t-)}(\xi(t)-\xi(t-))^{\alpha} & \text { if } \xi \in \tilde{\mathbb{D}}_{\leqslant \infty} \\ \infty, & \text { otherwise }\end{cases}
$$

Theorem 2.2. $\quad \bar{S}_{n}$ satisfies the extended large deviation principle in $\left(\mathbb{D}, \mathcal{T}_{J_{1}}\right)$ with speed $L(n) n^{\alpha}$ and rate function $\tilde{I}$.

Similarly to the case of Lévy processes, the proof of Theorem 2.2 is facilitated by Lemma 2.4, Lemma 2.5, and Lemma 2.6; their proofs are deferred to Section 5.6. Let $\tilde{Q}^{\leftarrow}(x)=\inf \{y \geq 0$ : $\mathbf{P}(Z \geq y)<x\}$. Set

$$
\tilde{J}_{n}^{k}(t) \triangleq \frac{1}{n} \sum_{i=1}^{k} \tilde{Q}^{\leftarrow}\left(V_{(i)}\right) \mathbb{1}_{\left[U_{i}, 1\right]}(t)+\frac{1}{n} Z \mathbb{1}_{\{1\}}(t)
$$

and

$$
\tilde{H}_{n}^{k}(t) \triangleq \frac{1}{n} \sum_{i=k+1}^{n-1} \tilde{Q}^{\leftarrow}\left(V_{(i)}\right) \mathbb{1}_{\left[U_{i}, 1\right]}(t)-\frac{1}{n} \mathbf{E} Z \sum_{i=1}^{n-1} \mathbb{1}_{\left[U_{i}, 1\right]}(t)-\frac{1}{n} \mathbf{E} Z \mathbb{1}_{\{1\}}(t)
$$


where $V_{(1)}, V_{(2)}, \ldots, V_{(n-1)}$ are the order statistics (in ascending order) of $V_{1}, V_{2}, \ldots, V_{n-1}$, which are i.i.d. Uniform $[0,1]$ and independent of $Z$. (In the proof of Theorem 2.2, we will show that $\tilde{S}_{n} \triangleq \tilde{J}_{n}^{k}+\tilde{H}_{n}^{k}$ satisfies the extended LDP with speed $L(n) n^{\alpha}$ and is exponentially equivalent to $\bar{S}_{n}$ so that Corollary 2.1 applies, and hence, in turn, $\bar{S}_{n}$ satisfies the same extended LDP.) Let $\tilde{I}_{k}$ be defined as follows:

$$
\tilde{I}_{k}(\xi)= \begin{cases}\sum_{t: \xi(t) \neq \xi(t-)}(\xi(t)-\xi(t-))^{\alpha} & \text { if } \xi \in \tilde{\mathbb{D}}_{\leqslant k} \\ \infty & \text { otherwise }\end{cases}
$$

LEMMA 2.4. For each fixed $k, \tilde{J}_{n}^{k}$ satisfies the $L D P$ in $\left(\mathbb{D}, \mathcal{T}_{J_{1}}\right)$ with speed $L(n) n^{\alpha}$ and the rate function $\tilde{I}_{k}$.

Lemma 2.5. For each $\delta>0$ and each open set $G$, there exist $\epsilon>0$ and $K \geq 0$ such that for any $k \geq K$

$$
\inf _{\xi \in G^{-\epsilon}} \tilde{I}_{k}(\xi) \leq \inf _{\xi \in G} \tilde{I}(\xi)+\delta
$$

Lemma 2.6. For every $\epsilon>0$ it holds that

$$
\limsup _{k \rightarrow \infty} \frac{1}{L(n) n^{\alpha}} \log \mathbf{P}\left(\left\|\tilde{H}_{n}^{k}\right\|_{\infty}>\epsilon\right)=-\infty
$$

With these lemmas in our hands, we are ready to prove Theorem 2.2.

Proof of Theorem 2.2. Let $\tilde{R}_{i} \triangleq\left|\left\{j \in \mathbb{N}: U_{j} \leq U_{i}, 1 \leq j \leq n-1\right\}\right|$. Then, we claim that

$$
\bar{S}_{n} \stackrel{\mathcal{D}}{=} \frac{1}{n} \sum_{i=1}^{n-1}\left(\tilde{Q}^{\leftarrow}\left(V_{(i)}\right)-\mathbf{E} Z\right) \mathbb{1}_{\left[\tilde{R}_{i} / n, 1\right]}+\frac{1}{n}(Z-\mathbf{E} Z) \mathbb{1}_{\{1\}}
$$

To see why this distributional equality holds, note that $\left\{\tilde{R}_{1}, \ldots, \tilde{R}_{n-1}\right\}$ is a uniformly random permutation of $\{1, \ldots, n-1\}$, and $\left\{\tilde{Q}^{\leftarrow}\left(V_{(1)}\right), \ldots, \tilde{Q}^{\leftarrow}\left(V_{(n-1)}\right)\right\}$ has the same distribution as the order statistics (in descending order) of $Z_{1}, \ldots, Z_{n-1}$ since $\tilde{Q}^{\leftarrow}\left(V_{i}\right)$ has the same distribution as $Z$ for each $i$. Now, we move on to showing that $\bar{S}_{n}$ is close to $\tilde{S}_{n}$-i.e., $\mathbf{P}\left(d_{J_{1}}\left(\tilde{S}_{n}, \bar{S}_{n}\right)>\epsilon\right)$ is asymptotically negligible. Recall that

$$
\tilde{S}_{n}=\frac{1}{n} \sum_{i=1}^{n-1}\left(\tilde{Q}^{\leftarrow}\left(V_{(i)}\right)-\mathbf{E} Z\right) \mathbb{1}_{\left[U_{i}, 1\right]}+\frac{1}{n}(Z-\mathbf{E} Z) \mathbb{1}_{\{1\}} .
$$

First observe that $\tilde{R}_{i}$ is the rank of $U_{i}$ among $U_{1}, \ldots, U_{n-1}$, and hence, $\tilde{R}_{i}^{\text {th }}$ earliest jump of $\bar{S}_{n}$ and $\tilde{S}_{n}$ are both $\tilde{Q}^{\leftarrow}\left(V_{(i)}\right)$. Therefore, the jumps associated with $\tilde{Q}^{\leftarrow}\left(V_{(1)}\right), \ldots, \tilde{Q}^{\leftarrow}\left(V_{(n-1)}\right), Z$ are arranged in the same order for $\bar{S}_{n}$ and $\tilde{S}_{n}$ with probability 1 . Moreover, the jump times of $\bar{S}_{n}$ and $\tilde{S}_{n}$ are $\frac{1}{n}, \frac{2}{n}, \ldots, \frac{n-1}{n}, \frac{n}{n}$ and $U_{(1)}, U_{(2)}, \ldots, U_{(n-1)}, 1$, respectively. Since $0<U_{(1)}<\cdots<U_{(n-1)}<$ 1 with probability 1 , the piecewise linear time change $\lambda:[0,1] \rightarrow[0,1]$ defined by the linear 
interpolation of $\lambda(0)=0, \lambda(1)=1$, and $\lambda(i / n)=U_{(i)}$ for $i=1, \ldots, n-1$ is a homeomorphism with probability 1 . Therefore, the $J_{1}$ distance between $\tilde{S}_{n}$ and $\bar{S}_{n}$ is bounded by

$$
\sup _{1 \leq i \leq n-1}\left|i / n-U_{(i)}\right|
$$

with probability 1 . On the other hand, by the sample path LDP for the order statistics of uniform random variables (Duffy, Macci and Torrisi, 2011), $\mathbf{P}\left(\sup _{1 \leq i \leq n-1}\left|i / n-U_{(i)}\right|>\epsilon\right)$ decays at an exponential rate, and hence,

$$
\limsup _{n \rightarrow \infty} \frac{1}{L(n) n^{\alpha}} \log \mathbf{P}\left(d_{J_{1}}\left(\tilde{S}_{n}, \bar{S}_{n}\right)>\epsilon\right) \leq \limsup _{n \rightarrow \infty} \frac{1}{L(n) n^{\alpha}} \log \mathbf{P}\left(\sup _{1 \leq i \leq n-1}\left|i / n-U_{(i)}\right|>\epsilon\right)=-\infty .
$$

In view of Corollary 2.1, the proof is done if we show that $\tilde{S}_{n}$ satisfies the extended LDP with speed $L(n) n^{\alpha}$ and the rate function $\tilde{I}$. To do so, we apply Proposition 2.1. Note that Lemma 2.4 verifies condition (i) of Proposition 2.1; (ii) is trivially satisfied since $\tilde{I}_{k} \geq \tilde{I}$; Lemma 2.5 verifies (iii); Lemma 2.6 verifies (iv). Therefore Proposition 2.1 applies to $\tilde{J}_{n}^{k}+\tilde{H}_{n}^{k}$, and the proof of Theorem 2.2 is complete.

2.3. Multi-dimensional processes. Let $X^{(1)}, \ldots, X^{(d)}$ be independent processes satisfying assumptions $A 1$ and $A 2$. Let $\bar{X}_{n}^{(i)}(t)$ denote the centered and scaled processes:

$$
\bar{X}_{n}^{(i)}(t) \triangleq \frac{1}{n} X^{(i)}(n t)-t \mathbf{E} X^{(i)}(1) .
$$

The next theorem establishes the extended LDP for $\left(\bar{X}_{n}^{(1)}, \ldots, \bar{X}_{n}^{(d)}\right)$.

Theorem 2.3. $\quad\left(\bar{X}_{n}^{(1)}, \bar{X}_{n}^{(2)}, \ldots, \bar{X}_{n}^{(d)}\right)$ satisfies the extended LDP in $\left(\prod_{i=1}^{d} \mathbb{D}\left([0,1], \mathbb{R}_{+}\right), \prod_{i=1}^{d} \mathcal{T}_{J_{1}}\right)$ with speed $L(n) n^{\alpha}$ and the rate function

$$
I^{d}\left(\xi_{1}, \ldots, \xi_{d}\right)= \begin{cases}\sum_{j=1}^{d} \sum_{t \in[0,1]}\left(\xi_{j}(t)-\xi_{j}(t-)\right)^{\alpha} & \text { if } \xi_{j} \in \mathbb{D}_{\leqslant \infty} \text { for each } j=1, \ldots, d, \\ \infty, & \text { otherwise }\end{cases}
$$

For each $i$, we consider the same distributional decomposition of $\bar{X}_{n}^{(i)}$ as in Section 2.1:

$$
\bar{X}_{n}^{(i)} \stackrel{\mathcal{D}}{=} \bar{J}_{n}^{k(i)}+\bar{H}_{n}^{k(i)}+\bar{R}_{n}^{(i)} .
$$

The proof of the theorem is immediate as in the one dimensional case, from Proposition 2.1, Lemma 2.3, and the following lemmas that parallel Lemma 2.1 and Lemma 2.2.

LEMMA 2.7. $\quad\left(\bar{J}_{n}^{k(1)}, \ldots, \bar{J}_{n}^{k(d)}\right)$ satisfy the LDP in $\left(\prod_{i=1}^{d} \mathbb{D}, \prod_{i=1}^{d} \mathcal{T}_{J_{1}}\right)$ with speed $L(n) n^{\alpha}$ and the rate function $I_{k}^{d}: \prod_{i=1}^{d} \mathbb{D} \rightarrow[0, \infty]$

$$
I_{k}^{d}\left(\xi_{1}, \ldots, \xi_{d}\right) \triangleq \begin{cases}\sum_{i=1}^{d} \sum_{t \in[0,1]}\left(\xi_{i}(t)-\xi_{i}(t-)\right)^{\alpha} & \text { if } \xi_{i} \in \mathbb{D}_{\leqslant k} \text { for } i=1, \ldots, d \\ \infty & \text { otherwise }\end{cases}
$$


Lemma 2.8. For each $\delta>0$ and each open set $G$, there exists $\epsilon>0$ and $K \geq 0$ such that for any $k \geq K$

$$
\inf _{\left(\xi_{1}, \ldots, \xi_{d}\right) \in G^{-\epsilon}} I_{k}^{d}\left(\xi_{1}, \ldots, \xi_{d}\right) \leq \inf _{\left(\xi_{1}, \ldots, \xi_{d}\right) \in G} I^{d}\left(\xi_{1}, \ldots, \xi_{d}\right)+\delta .
$$

We conclude this section with the extended LDP for multidimensional random walks. Let $S_{n}^{(i)}=Z_{1}^{(i)}+\cdots+Z_{n}^{(i)}$ be a random walk with non-negative increments for each $i=1, \ldots, d$. Consider $\bar{S}_{n}^{(i)}=\left\{\bar{S}_{n}^{(i)}(t), t \in[0,1]\right\}$ where $\bar{S}_{n}^{(i)}(t)=\frac{1}{n} \sum_{j=1}^{[n t]}\left(Z_{j}^{(i)}-\mathbf{E} Z^{(i)}\right)$. We assume that $\mathbf{P}\left(Z_{j}^{(i)} \geq x\right)=\exp \left(-L(x) x^{\alpha}\right)$ where $\alpha \in(0,1)$ and $L(\cdot)$ is a slowly varying function, and $A 2$ is in force. The following theorem can be obtained by "lifting" Lemma 2.4 and Lemma 2.5 to the multi-dimensional context - in the same way Lemma 2.1 and Lemma 2.2 were lifted to the multi-dimensional counterparts in the proof of Theorem 2.3 - and then applying Proposition 2.1. Let

$$
\tilde{I}^{d}\left(\xi_{1}, \ldots, \xi_{d}\right)= \begin{cases}\sum_{j=1}^{d} \sum_{t \in[0,1]}\left(\xi_{j}(t)-\xi_{j}(t-)\right)^{\alpha} & \text { if } \xi_{j} \in \tilde{\mathbb{D}}_{\leqslant \infty} \text { for each } j=1, \ldots, d \\ \infty & \text { otherwise }\end{cases}
$$

THEOREM 2.4. $\left(\bar{S}_{n}^{(1)}, \bar{S}_{n}^{(2)}, \ldots, \bar{S}_{n}^{(d)}\right)$ satisfies the extended LDP in $\left(\prod_{i=1}^{d} \mathbb{D}, \prod_{i=1}^{d} \mathcal{T}_{J_{1}}\right)$ with speed $L(n) n^{\alpha}$ and the rate function $\tilde{I}^{d}$.

REMARK 2.2. Note that Theorem 2.3 and Theorem 2.4 can be extended to heterogeneous processes. For example, if the Lévy measure $\nu^{(i)}$ of the process $X^{(i)}$ has Weibull tail distribution $\nu^{(i)}[x, \infty)=\exp \left(-c_{i} L(x) x^{\alpha}\right)$ where $c_{i} \in(0, \infty)$ for each $i \leq d_{0} \leq d$, and all the other processes have lighter tails-i.e., $L(x) x^{\alpha}=o\left(L_{i}(x) x^{\alpha_{i}}\right)$ for $i>d_{0}$-then it is straightforward to check that $\left(\bar{X}_{n}^{(1)}, \ldots, \bar{X}_{n}^{(d)}\right)$ satisfies the extended LDP with the rate function

$$
I^{d}\left(\xi_{1}, \ldots, \xi_{d}\right)= \begin{cases}\sum_{j=1}^{d_{0}} c_{j} \sum_{t \in[0,1]}\left(\xi_{j}(t)-\xi_{j}(t-)\right)^{\alpha} & \text { if } \xi_{j} \in \mathbb{D}_{\leqslant \infty}[0,1] \text { for } j=1, \ldots, d_{0} \\ \infty & \text { and } \xi_{j} \equiv 0 \text { for } j>d_{0} \\ \text { otherwise } .\end{cases}
$$

Similarly, $\left(\bar{S}_{n}^{(1)}, \ldots, \bar{S}_{n}^{(d)}\right)$ satisfies the extended LDP with the rate function $\tilde{I}^{d}$ defined by replacing $\mathbb{D}_{\leqslant \infty}$ with $\tilde{\mathbb{D}}_{\leqslant \infty}$ in the definition of $I^{d}$ above under the corresponding conditions on the tail distribution of $Z_{1}^{(i)}$ 's.

\section{Implications and further discussions.}

3.1. LDP for Lipschitz functions of Lévy processes. Let $\overline{\mathbf{X}}_{n}$ denote the scaled Lévy processes $\left(\bar{X}_{n}^{(1)}, \ldots, \bar{X}_{n}^{(d)}\right)$, and $\overline{\mathbf{S}}_{n}$ denote the scaled random walks $\left(\bar{S}_{n}^{(1)}, \ldots, \bar{S}_{n}^{(d)}\right)$ as defined in Section 2. Recall also the rate functions $I^{d}$ defined in (2.11) and $\tilde{I}^{d}$ defined in (2.14). 
COROllary 3.1. Let $(\mathbb{S}, d)$ be a metric space and $\phi: \prod_{i=1}^{d} \mathbb{D} \rightarrow \mathbb{S}$ be a Lipschitz continuous mapping w.r.t. the $J_{1}$ Skorokhod metric. Set

$$
I^{\prime}(x) \triangleq \inf _{\phi(\xi)=x} I^{d}(\xi) \quad \text { and } \quad \tilde{I}^{\prime}(x) \triangleq \inf _{\phi(\xi)=x} \tilde{I}^{d}(\xi)
$$

and suppose that $I^{\prime}$ (or $\left.\tilde{I}^{\prime}\right)$ is a good rate function-i.e., $\Psi_{I^{\prime}}(a) \triangleq\left\{x \in \mathbb{S}: I^{\prime}(s) \leq a\right\}$ (or $\left.\Psi_{\tilde{I}^{\prime}}(a) \triangleq\left\{x \in \mathbb{S}: \tilde{I}^{\prime}(s) \leq a\right\}\right)$ is compact for each $a \in[0, \infty)$. Then, $\phi\left(\overline{\mathbf{X}}_{n}\right)$ (or $\left.\phi\left(\overline{\mathbf{S}}_{n}\right)\right)$ satisfies the large deviation principle in $(\mathbb{S}, d)$ with speed $L(n) n^{\alpha}$ and the good rate function $I^{\prime}$ (or $\tilde{I}^{\prime}$ ).

Proof. Since the argument for $\phi\left(\overline{\mathbf{S}}_{n}\right)$ is very similar, we only prove the LDP for $\phi\left(\overline{\mathbf{X}}_{n}\right)$. We start with the upper bound. Suppose that the Lipschitz constant of $\phi$ is $\|\phi\|_{\text {Lip. Note that since }}$ the $J_{1}$ distance is dominated by the supremum distance, $\left\|\overline{\mathbf{H}}_{n}^{k}\right\|_{\infty} \leq \epsilon$ and $\left\|\overline{\mathbf{R}}_{n}\right\|_{\infty} \leq \epsilon$ implies $d_{J_{1}}\left(\phi\left(\overline{\mathbf{J}}_{n}^{k}\right), \phi\left(\overline{\mathbf{X}}_{n}\right)\right) \leq 2 \epsilon\|\phi\|_{\text {Lip }}$, where $\overline{\mathbf{J}}_{n}^{k} \triangleq\left(\bar{J}_{n}^{k(1)}, \ldots, \bar{J}_{n}^{k(d)}\right), \overline{\mathbf{H}}_{n}^{k} \triangleq\left(\bar{H}_{n}^{k(1)}, \ldots, \bar{H}_{n}^{k(d)}\right)$, and $\overline{\mathbf{R}}_{n} \triangleq\left(\bar{R}_{n}^{(1)}, \ldots, \bar{R}_{n}^{(d)}\right)$. Therefore, for any closed set $F$,

$$
\begin{aligned}
& \mathbf{P}\left(\phi\left(\overline{\mathbf{X}}_{n}\right) \in F\right) \\
& \leq \mathbf{P}\left(\phi\left(\overline{\mathbf{X}}_{n}\right) \in F, d_{J_{1}}\left(\phi\left(\overline{\mathbf{J}}_{n}^{k}\right), \phi\left(\overline{\mathbf{X}}_{n}\right)\right) \leq 2 \epsilon\|\phi\|_{\text {Lip }}\right)+\mathbf{P}\left(d_{J_{1}}\left(\phi\left(\overline{\mathbf{J}}_{n}^{k}\right), \phi\left(\overline{\mathbf{X}}_{n}\right)\right)>2 \epsilon\|\phi\|_{\text {Lip }}\right) \\
& \leq \mathbf{P}\left(\phi\left(\overline{\mathbf{J}}_{n}^{k}\right) \in F^{2 \epsilon\|\phi\|_{\text {Lip }}}\right)+\mathbf{P}\left(d_{J_{1}}\left(\phi\left(\overline{\mathbf{J}}_{n}^{k}\right), \phi\left(\overline{\mathbf{X}}_{n}\right)\right)>2 \epsilon\|\phi\|_{\text {Lip }}\right) \\
& \leq \mathbf{P}\left(\overline{\mathbf{J}}_{n}^{k} \in \phi^{-1}\left(F^{2 \epsilon\|\phi\|_{\text {Lip }}}\right)\right)+\mathbf{P}\left(\left\|\overline{\mathbf{H}}_{n}^{k}\right\|_{\infty}>\epsilon\right)+\mathbf{P}\left(\left\|\overline{\mathbf{R}}_{n}\right\|_{\infty}>\epsilon\right) .
\end{aligned}
$$

Since $\mathbf{P}\left(\left\|\overline{\mathbf{R}}_{n}\right\|_{\infty}>\epsilon\right)$ decays at an exponential rate and $\mathbf{P}\left(\left\|\overline{\mathbf{H}}_{n}^{k}\right\|_{\infty}>\epsilon\right) \leq \sum_{i=1}^{d} \mathbf{P}\left(\left\|\bar{H}_{n}^{k(i)}\right\|_{\infty}>\right.$ $\epsilon / d)$, we get the following bound by applying the principle of the maximum term and Theorem 2.3:

$$
\begin{aligned}
& \limsup _{n \rightarrow \infty} \frac{1}{L(n) n^{\alpha}} \log \mathbf{P}\left(\phi\left(\overline{\mathbf{X}}_{n}\right) \in F\right) \\
& \leq \limsup _{n \rightarrow \infty} \frac{1}{L(n) n^{\alpha}} \log \left\{\mathbf{P}\left(\overline{\mathbf{J}}_{n}^{k} \in \phi^{-1}\left(F^{2 \epsilon\|\phi\|_{\text {Lip }}}\right)\right)+\mathbf{P}\left(\left\|\overline{\mathbf{H}}_{n}^{k}\right\|_{\infty}>\epsilon\right)+\mathbf{P}\left(\left\|\overline{\mathbf{R}}_{n}\right\|_{\infty}>\epsilon\right)\right\} \\
& =\max \left\{\limsup _{n \rightarrow \infty} \frac{\log \mathbf{P}\left(\overline{\mathbf{J}}_{n}^{k} \in \phi^{-1}\left(F^{2 \epsilon\|\phi\|_{\text {Lip }}}\right)\right)}{L(n) n^{\alpha}}, \limsup _{n \rightarrow \infty} \frac{\log \mathbf{P}\left(\left\|\overline{\mathbf{H}}_{n}^{k}\right\|_{\infty}>\epsilon\right)}{L(n) n^{\alpha}}\right\} \\
& \leq \max \left\{-\inf _{\left(\xi_{1}, \ldots, \xi_{d}\right) \in \phi^{-1}\left(F^{2 \epsilon\|\phi\|_{\text {Lip }}}\right)} I_{k}^{d}\left(\xi_{1}, \ldots, \xi_{d}\right), \max _{i=1, \ldots, d} \limsup _{n \rightarrow \infty} \frac{\log \mathbf{P}\left(\left\|\bar{H}_{n}^{k(i)}\right\|_{\infty}>\epsilon / d\right)}{L(n) n^{\alpha}}\right\} \\
& \leq \max \left\{-\inf _{\left(\xi_{1}, \ldots, \xi_{d}\right) \in \phi^{-1}\left(F^{2 \epsilon\|\phi\|_{\text {Lip }}}\right)} I^{d}\left(\xi_{1}, \ldots, \xi_{d}\right), \limsup _{n \rightarrow \infty} \frac{\log \mathbf{P}\left(\left\|\bar{H}_{n}^{k(1)}\right\|_{\infty}>\epsilon / d\right)}{L(n) n^{\alpha}}\right\} .
\end{aligned}
$$

From Lemma 2.3, we can take $k \rightarrow \infty$ to get

$$
\limsup _{n \rightarrow \infty} \frac{\log \mathbf{P}\left(\phi\left(\overline{\mathbf{X}}_{n}\right) \in F\right)}{L(n) n^{\alpha}} \leq-\inf _{\left(\xi_{1}, \ldots, \xi_{d}\right) \in \phi^{-1}\left(F^{2 \epsilon\|\phi\|_{\text {Lip }}}\right)} I^{d}\left(\xi_{1}, \ldots, \xi_{d}\right)=-\inf _{x \in F^{2 \epsilon\|\phi\|_{\text {Lip }}}} I^{\prime}(x)
$$


From Lemma 4.1.6 of Dembo and Zeitouni (2010), $\lim _{\epsilon \rightarrow 0} \inf _{x \in F^{\epsilon}\|\phi\|_{\text {Lip }}} I^{\prime}(x)=\inf _{x \in F} I^{\prime}(x)$. Letting $\epsilon \rightarrow 0$ in (3.1), we arrive at the desired large deviation upper bound.

Turning to the lower bound, consider an open set $G$. Since $\phi^{-1}(G)$ is open, from Theorem 2.3,

$$
\begin{aligned}
\liminf _{n \rightarrow \infty} \frac{1}{L(n) n^{\alpha}} \log \mathbf{P}\left(\phi\left(\overline{\mathbf{X}}_{n}\right) \in G\right) & =\liminf _{n \rightarrow \infty} \frac{1}{L(n) n^{\alpha}} \log \mathbf{P}\left(\overline{\mathbf{X}}_{n} \in \phi^{-1}(G)\right) \\
& \geq-\inf _{\left(\xi_{1}, \ldots, \xi_{d}\right) \in \phi^{-1}(G)} I(\xi)=-\inf _{x \in G} I^{\prime}(x) .
\end{aligned}
$$

3.2. Sample path LDP w.r.t. $M_{1}^{\prime}$ topology. Recall that $\bar{X}_{n}(t) \triangleq \frac{1}{n} X(n t)-t \mathbf{E} X(1)$ and $\bar{S}_{n}(t)=$ $\frac{1}{n} \sum_{i=1}^{[n t]}\left(Z_{i}-\mathbf{E} Z\right)$. In this section, we establish the full LDP for $\bar{X}_{n}$ and $\bar{S}_{n}$ w.r.t. the $M_{1}^{\prime}$ topology. For the definition of the $M_{1}^{\prime}$ topology, see Appendix A.

Corollary 3.2. $\quad \bar{X}_{n}$ and $\bar{S}_{n}$ satisfy the $L D P$ in $\left(\mathbb{D}, \mathcal{T}_{M_{1}^{\prime}}\right)$ with speed $L(n) n^{\alpha}$ and the good rate function $I_{M_{1}^{\prime}}$.

$$
I_{M_{1}^{\prime}}(\xi) \triangleq \begin{cases}\sum_{t \in[0,1]}(\xi(t)-\xi(t-))^{\alpha} & \text { if } \xi \text { is a non-decreasing pure jump function with } \xi(0) \geq 0 \\ \infty & \text { otherwise. }\end{cases}
$$

Proof. Since the proof for $\bar{S}_{n}$ is nearly identical, we only provide the proof for $\bar{X}_{n}$. From Proposition A.3 we know that $I_{M_{1}^{\prime}}$ is a good rate function. For the LDP upper bound, suppose that $F$ is a closed set w.r.t. the $M_{1}^{\prime}$ topology. Then, it is also closed w.r.t. the $J_{1}$ topology. From the upper bound of Theorem 2.1 and the fact that $I_{M_{1}^{\prime}}(\xi) \leq I(\xi)$ for any $\xi \in \mathbb{D}$,

$$
\limsup _{n \rightarrow \infty} \frac{\log \mathbf{P}\left(\bar{X}_{n} \in F\right)}{L(n) n^{\alpha}} \leq-\lim _{\epsilon \rightarrow 0} \inf _{\xi \in F^{\epsilon}} I(\xi) \leq-\lim _{\epsilon \rightarrow 0} \inf _{\xi \in F^{\epsilon}} I_{M_{1}^{\prime}}(\xi)=-\inf _{\xi \in F} I_{M_{1}^{\prime}}(\xi) .
$$

Turning to the lower bound, suppose that $G$ is an open set w.r.t. the $M_{1}^{\prime}$ topology. We claim that

$$
\inf _{\xi \in G} I_{M_{1}^{\prime}}(\xi)=\inf _{\xi \in G} I(\xi) .
$$

To show this, we only have to show that the RHS is not strictly larger than the LHS. Suppose that $I_{M_{1}^{\prime}}(\xi)<I(\xi)$ for some $\xi \in G$. Since $I$ and $I_{M_{1}^{\prime}}$ differ only if the path has a jump at either 0 or 1 , this means that $\xi$ is a non-negative pure jump function of the following form:

$$
\xi=\sum_{i=1}^{\infty} z_{i} \mathbb{1}_{\left[u_{i}, 1\right]}
$$

where $u_{1}=0, u_{2}=1, u_{i}$ 's are all distinct in $(0,1)$ for $i \geq 3$ and $z_{i} \geq 0$ for all $i$ 's. Note that one can pick an arbitrarily small $\epsilon$ so that $\sum_{i \in\left\{n: u_{n}<\epsilon\right\}} z_{i}<\epsilon, \sum_{i \in\left\{n: u_{n}>1-\epsilon\right\}} z_{i}<\epsilon, \epsilon \neq u_{i}$ for all $i \geq 2$, and $1-\epsilon \neq u_{i}$ for all $i \geq 2$. For such $\epsilon$ 's, if we set

$$
\xi_{\epsilon} \triangleq z_{1} \mathbb{1}_{[\epsilon, 1]}+z_{2} \mathbb{1}_{[1-\epsilon, 1]}+\sum_{i=3}^{\infty} z_{i} \mathbb{1}_{\left[u_{i}, 1\right]}
$$


then $d_{M_{1}^{\prime}}\left(\xi, \xi_{\epsilon}\right) \leq \epsilon$ while $I\left(\xi_{\epsilon}\right)=I_{M_{1}^{\prime}}(\xi)$. That is, we can find an arbitrarily close element $\xi_{\epsilon}$ from $\xi$ w.r.t. the $M_{1}^{\prime}$ metric by pushing the jump times at 0 and 1 slightly to the inside of $(0,1)$; at such an element, $I$ assumes the same value as $I_{M_{1}^{\prime}}(\xi)$. Since $G$ is open w.r.t. $M_{1}^{\prime}$, one can choose $\epsilon$ small enough so that $\xi_{\epsilon} \in G$. This proves the claim. Now, the desired LDP lower bound is immediate from the LDP lower bound in Theorem 2.1 since $G$ is also an open set in the $J_{1}$ topology.

3.3. Nonexistence of large deviation principle in the $J_{1}$ topology. Consider a compound Poisson process with arrival rate equal to 1 whose jump distribution is Weibull with the shape parameter $1 / 2$. More specifically, $\bar{X}_{n}(t) \triangleq \frac{1}{n} \sum_{i=1}^{N(n t)} Z_{i}-t$ with $\mathbf{P}\left(Z_{i} \geq x\right) \sim \exp \left(-x^{\alpha}\right), \mathbf{E} Z_{i}=1$, and $\alpha=1 / 2$. If $\bar{X}_{n}$ satisfies a full LDP w.r.t. the $J_{1}$ topology, the rate function that controls the LDP (with speed $n^{\alpha}$ ) associated with $\bar{X}_{n}$ should be of the same form as the one that controls the extended LDP:

$$
I(\xi)= \begin{cases}\sum_{t \in[0,1]}(\xi(t)-\xi(t-))^{\alpha} & \text { if } \xi \in \mathbb{D}_{\leqslant \infty}, \\ \infty, & \text { otherwise. }\end{cases}
$$

To show that such a LDP is fundamentally impossible, we construct a closed set $A$ for which

$$
\limsup _{n \rightarrow \infty} \frac{\log \mathbf{P}\left(\bar{X}_{n} \in A\right)}{n^{\alpha}}>-\inf _{\xi \in A} I(\xi)
$$

Let

$$
\varphi_{s, t}(\xi) \triangleq \lim _{\epsilon \rightarrow 0} \sup _{0 \vee(s-\epsilon) \leq u \leq v \leq 1 \wedge(t+\epsilon)}(\xi(v)-\xi(u)) .
$$

Let $A_{c ; s, t} \triangleq\left\{\xi: \varphi_{s, t}(\xi) \geq c\right\}$ be (roughly speaking) the set of paths which increase at least by $c$ between time $s$ and $t$. Then $A_{c ; s, t}$ is a closed set for each $c, s$, and $t$.

Let

$$
A_{m} \triangleq\left(A_{1 ; \frac{m+1}{m+2}, \frac{m+1}{m+2}+m h_{m}}\right) \cap\left(A_{1 ; \frac{m}{m+2}, \frac{m}{m+2}+m h_{m}}\right) \cap\left(\bigcap_{j=0}^{m-1} A_{\frac{1}{m^{2}} ; \frac{j}{m+2}, \frac{j}{m+2}+m h_{m}}\right)
$$

where $h_{m}=\frac{1}{(m+1)(m+2)}$, and let

$$
A \triangleq \bigcup_{m=1}^{\infty} A_{m}
$$

To see that $A$ is closed, we first claim that $\zeta \in \mathbb{D} \backslash A$ implies the existence of $\epsilon>0$ and $N \geq 0$ such that $B(\zeta ; \epsilon) \cap A_{m}=\emptyset$ for all $m \geq N$. To prove this claim, suppose not. It is straightforward to check that for each $n$, there has to be $s_{n}, t_{n} \in[1-1 / n, 1)$ such that $s_{n} \leq t_{n}$ and $\zeta\left(t_{n}\right)-\zeta\left(s_{n}\right) \geq 1 / 2$, which in turn implies that $\zeta$ must possess infinite number of increases of size at least $1 / 2$ in $[1-\delta, 1)$ for any $\delta>0$. This implies that $\zeta$ cannot possess a left limit, which is contradictory to the assumption that $\zeta \in \mathbb{D} \backslash A$. On the other hand, since each $A_{m}$ is closed, $\bigcup_{i=1}^{N} A_{i}$ is also closed, and hence, there exists $\epsilon^{\prime}>0$ such that $B\left(\zeta ; \epsilon^{\prime}\right) \cap A_{m}=\emptyset$ for $m=1, \ldots, N$. Now, from the construction of $\epsilon$ and $\epsilon^{\prime}, B\left(\zeta, \epsilon \vee \epsilon^{\prime}\right) \cap A=\emptyset$, proving that $A$ is closed. 
Next, we show that $A$ satisfies (3.2). First note that if $\xi$ is a pure jump function that belongs to $A_{m}, \xi$ has to possess $m$ upward jumps of size $1 / m^{2}$ and 2 upward jumps of size 1 , and hence,

$$
\inf _{\xi \in A} I(\xi) \geq \inf _{m}\left(1^{1 / 2}+1^{1 / 2}+m\left(1 / m^{2}\right)^{1 / 2}\right)=3 .
$$

On the other hand, letting $\Delta \xi(t) \triangleq \xi(t)-\xi(t-)$,

$$
\begin{aligned}
\mathbf{P}\left(\bar{X}_{(n+1)(n+2)} \in A_{n}\right) \\
\geq \prod_{j=0}^{n-1} \mathbf{P}\left(\sup _{t \in[0,1]}\left\{\bar{X}_{(n+1)(n+2)}\left(\frac{(n+1) j+n t}{(n+1)(n+2)}\right)-\bar{X}_{(n+1)(n+2)}\left(\frac{(n+1) j}{(n+1)(n+2)}\right)\right\} \geq \frac{1}{n^{2}}\right) \\
\quad \cdot \mathbf{P}\left(\sup _{t \in(0,1]}\left\{\Delta \bar{X}_{(n+1)(n+2)}\left(\frac{(n+1) n+n t}{(n+1)(n+2)}\right)\right\} \geq 1\right) \cdot \mathbf{P}\left(\sup _{t \in(0,1]}\left\{\Delta \bar{X}_{(n+1)(n+2)}\left(\frac{(n+1)(n+1)+n t}{(n+1)(n+2)}\right)\right\} \geq 1\right) \\
=\mathbf{P}\left(\sup _{t \in[0,1]}\left\{\bar{X}_{(n+1)(n+2)}\left(\frac{n t}{(n+1)(n+2)}\right)\right\} \geq \frac{1}{n^{2}}\right)^{n} \cdot \mathbf{P}\left(\sup _{t \in[0,1]}\left\{\Delta \bar{X}_{(n+1)(n+2)}\left(\frac{n t}{(n+1)(n+2)}\right)\right\} \geq 1\right)^{2} \\
=\mathbf{P}\left(\sup _{t \in[0,1]}\{X(n t)\} \geq \frac{(n+1)(n+2)}{n^{2}}\right)^{n} \cdot \mathbf{P}\left(\sup _{t \in[0,1]}\{\Delta X(n t)\} \geq(n+1)(n+2)\right)^{2} \\
\geq \mathbf{P}\left(\sup _{t \in[0,1]}\{X(n t)\} \geq 6\right)^{n} \cdot \mathbf{P}\left(\sup _{t \in[0,1]}\{\Delta X(n t)\} \geq(n+1)(n+2)\right)^{2},
\end{aligned}
$$

and hence,

$$
\begin{aligned}
& \limsup _{n \rightarrow \infty} \frac{\log \mathbf{P}\left(\bar{X}_{n} \in A\right)}{n^{\alpha}} \geq \limsup _{n \rightarrow \infty} \frac{\log \mathbf{P}\left(\bar{X}_{(n+1)(n+2)} \in A_{n}\right)}{((n+1)(n+2))^{\alpha}} \\
& \geq \limsup _{n \rightarrow \infty} \frac{\log \mathbf{P}\left(\sup _{t \in[0,1]}\{X(n t)\} \geq 6\right)^{n}}{((n+1)(n+2))^{\alpha}}+2 \limsup _{n \rightarrow \infty} \frac{\log \mathbf{P}\left(\sup _{t \in[0,1]}\{\Delta X(n t)\} \geq(n+1)(n+2)\right)}{((n+1)(n+2))^{\alpha}} \\
& =(\mathrm{I})+(\mathrm{II}) .
\end{aligned}
$$

Letting $p_{n} \triangleq \mathbf{P}\left(\sup _{t \in[0, n]}\{X(t)\}<6\right)$,

$$
\text { (I) }=\limsup _{n \rightarrow \infty} \frac{\log \left(1-p_{n}\right)^{n}}{((n+1)(n+2))^{\alpha}}=\limsup _{n \rightarrow \infty} \frac{n p_{n} \log \left(1-p_{n}\right)^{1 / p_{n}}}{((n+1)(n+2))^{\alpha}}=\limsup _{n \rightarrow \infty} \frac{-n p_{n}}{((n+1)(n+2))^{\alpha}}=0
$$

since $p_{n} \rightarrow 0$ as $n \rightarrow \infty$.

For term (II), notice that that $\left\{Z_{1} \geq(n+1)(n+2)\right\} \Longrightarrow\left\{\sup _{t \in[0,1]}\{\Delta X(n t)\} \geq(n+1)(n+2)\right\}$. That is, 


$$
\begin{aligned}
(\mathrm{II}) & =2 \limsup _{n \rightarrow \infty} \frac{\log \mathbf{P}\left(\sup _{t \in[0,1]}\{\Delta X(n t)\} \geq(n+1)(n+2)\right)}{((n+1)(n+2))^{\alpha}} \\
& \geq 2 \limsup _{n \rightarrow \infty} \frac{\log \mathbf{P}\left(Z_{1} \geq(n+1)(n+2)\right)}{((n+1)(n+2))^{\alpha}}=2 \limsup _{n \rightarrow \infty} \frac{\log e^{-((n+1)(n+2))^{\alpha}}}{((n+1)(n+2))^{\alpha}} \\
& =-2 .
\end{aligned}
$$

From (3.4), (3.5), (3.6),

$$
\limsup _{n \rightarrow \infty} \frac{\log \mathbf{P}\left(\bar{X}_{n} \in A\right)}{n^{\alpha}} \geq-2
$$

This along with (3.3),

$$
\limsup _{n \rightarrow \infty} \frac{\log \mathbf{P}\left(\bar{X}_{n} \in A\right)}{n^{\alpha}} \geq-2>-3 \geq-\inf _{\xi \in A} I(\xi),
$$

which means that $A$ indeed is a counterexample for the desired LDP.

Note that a simpler counterexample can be constructed using the peculiarity of the $J_{1}$ topology at the boundary of the domain; that is, jumps (of size 1, say) at time 0 are bounded away from the jumps at arbitrarily close jump times. For example, if $\bar{Y}_{n}(t) \triangleq \frac{1}{n} \sum_{i=1}^{N(n t)} Z_{i}+t$ where the right tail of $Z$ is Weibull and $\mathbf{E} Z=-1$, then the set $B=\{x: x(t) \geq t / 2$ for all $t \in[0,1]\}$ gives a counterexample for the LDP. Note that the $M_{1}^{\prime}$ topology we used in Section 3.2 is a treatment for the same peculiarity of the $M_{1}$ topology at time 0 . However, it should be clear from the above counterexample $\bar{X}_{n}$ and $A$ that the LDP is fundamentally impossible w.r.t. $J_{1}$-like topologiesi.e., the ones that do not allow merging two or more jumps to approximate a single jump at any time - and hence, there is no hope for the full LDP in the case of the $J_{1}$ topology.

4. Boundary crossing with moderate jumps. In this section, we illustrate the value of Corollary 3.1; for an application of Corollary 3.2 we refer to Bazhba et al. (2019). We consider level crossing probabilities of Lévy processes where the jump sizes are conditioned to be moderate. Specifically, we apply Corollary 3.1 in order to provide large-deviations estimates for

$$
\mathbf{P}\left(\sup _{t \in[0,1]} \bar{X}_{n}(t) \geq c, \sup _{t \in[0,1]}\left|\bar{X}_{n}(t)-\bar{X}_{n}(t-)\right| \leq b\right) .
$$

We emphasize that this type of rare events are difficult to analyze with the tools developed previously. In particular, the sample path LDP proved in Gantert (1998) is w.r.t. the $L_{1}$ topology. Since the closure of the sets in (4.1) w.r.t. the $L_{1}$ topology contains the zero function, the LDP upper bound would not provide any information. 
Functionals like (4.1) appear in actuarial models, in case excessively large insurance claims are reinsured and therefore do not play a role in the ruin of an insurance company. Asmussen and Pihlsgård (2005), for example, studied various estimates of infinite-time ruin probabilities with analytic methods. Rhee, Blanchet and Zwart (2016) studied the finite-time ruin probability, using probabilistic techniques in case of regularly varying Lévy measures and confirmed that the conventional wisdom "the principle of a single big jump" can be extended to "the principle of the minimal number of big jumps" in such a context. Here we show that a similar result — with subtle differences - can be obtained in case the Lévy measure has a Weibull tail.

Define the function $\phi: \mathbb{D} \rightarrow \mathbb{R}^{2}$ as

$$
\phi(\xi)=\left(\phi_{1}(\xi), \phi_{2}(\xi)\right) \triangleq\left(\sup _{t \in[0,1]} \xi(t), \sup _{t \in[0,1]}|\xi(t)-\xi(t-)|\right) .
$$

In order to apply Corollary 3.1, we will validate that $\phi$ is $\operatorname{Lipschitz}$ continuous and that $I^{\prime}(x, y) \triangleq$ $\inf _{\{\xi \in \mathbb{D}: \phi(\xi)=(x, y)\}} I(\xi)$ is a good rate function.

For the Lipschitz continuity of $\phi$, we claim that each component of $\phi$ is Lipschitz continuous. We first examine $\phi_{1}$. Let $\xi, \zeta \in \mathbb{D}$ and suppose w.l.o.g. that $\sup _{t \in[0,1]} \xi(t)>\sup _{t \in[0,1]} \zeta(t)$. For an arbitrary non-decreasing homeomorphism $\lambda:[0,1] \rightarrow[0,1]$,

$$
\begin{aligned}
\left|\phi_{1}(\xi)-\phi_{1}(\zeta)\right| & =\left|\sup _{t \in[0,1]} \xi(t)-\sup _{t \in[0,1]} \zeta(t)\right|=\left|\sup _{t \in[0,1]} \xi(t)-\sup _{t \in[0,1]} \zeta \circ \lambda(t)\right| \\
& \leq \sup _{t \in[0,1]}|\xi(t)-\zeta \circ \lambda(t)| \leq \sup _{t \in[0,1]}|\xi(t)-\zeta \circ \lambda(t)| \vee|\lambda(t)-t| .
\end{aligned}
$$

Taking the infimum over $\lambda$, we conclude that

$$
\left|\phi_{1}(\xi)-\phi_{1}(\zeta)\right| \leq \inf _{\lambda \in \Lambda} \sup _{t \in[0,1]}\{|\xi(t)-\zeta(\lambda(t))| \vee|\lambda(t)-t|\}=d_{J_{1}}(\xi, \zeta) .
$$

Therefore, $\phi_{1}$ is Lipschitz with the Lipschitz constant 1.

Now, in order to prove that $\phi_{2}(\xi)$ is Lipschitz, fix two distinct paths $\xi, \zeta \in \mathbb{D}$ and assume w.l.o.g. that $\phi_{2}(\zeta)>\phi_{2}(\xi)$. Let $c \triangleq \phi_{2}(\zeta)-\phi_{2}(\xi)>0$, and let $t^{*} \in[0,1]$ be the maximum jump time of $\xi$, i.e., $\phi_{2}(\xi)=\left|\xi\left(t^{*}\right)-\xi\left(t^{*}-\right)\right|$. For any $\epsilon>0$ there exists $\lambda^{*}$ so that

$$
\begin{aligned}
d_{J_{1}}(\xi, \zeta) & \triangleq \inf _{\lambda \in \Lambda}\left\{\|\xi-\zeta \circ \lambda\|_{\infty} \vee\|\lambda-e\|_{\infty}\right\} \geq\left\|\xi-\zeta \circ \lambda^{*}\right\|_{\infty} \vee\left\|\lambda^{*}-e\right\|_{\infty}-\epsilon \\
& \geq\left|\xi\left(t^{*}\right)-\zeta \circ \lambda^{*}\left(t^{*}\right)\right| \vee\left|\xi\left(t^{*}-\right)-\zeta \circ \lambda^{*}\left(t^{*}-\right)\right|-\epsilon
\end{aligned}
$$

From the general inequality $|a-b| \vee|c-d| \geq \frac{1}{2}(|a-c|-|b-d|)$,

$$
\begin{aligned}
\left|\xi\left(t^{*}\right)-\zeta \circ \lambda^{*}\left(t^{*}\right)\right| \vee\left|\xi\left(t_{1^{-}}\right)-\zeta \circ \lambda^{*}\left(t^{*}-\right)\right| & \geq \frac{1}{2}\left(\left|\xi\left(t^{*}\right)-\xi\left(t^{*}-\right)\right|-\left|\zeta \circ \lambda^{*}\left(t^{*}\right)-\zeta \circ \lambda^{*}\left(t^{*}-\right)\right|\right) \\
& =\frac{1}{2}\left(\phi_{2}(\xi)-\phi_{2}(\zeta)\right)=c / 2
\end{aligned}
$$

In view of (4.3) and (4.4), $d_{J_{1}}(\xi, \zeta) \geq \frac{1}{2}|\phi(\xi)-\phi(\zeta)|-\epsilon$. Since $\epsilon$ is arbitrary, we get the desired Lipschitz bound with Lipschitz constant 2. Therefore, $|\phi(\xi)-\phi(\zeta)|=\left|\phi_{1}(\xi)-\phi_{1}(\zeta)\right| \vee \mid \phi_{2}(\xi)-$ $\phi_{2}(\zeta) \mid \leq 2 d_{J_{1}}(\xi, \zeta)$ and hence, $\phi$ is Lipschitz with Lipschitz constant 2. 
Now, we claim that $I^{\prime}$ is of the following form:

$$
I^{\prime}(c, b)= \begin{cases}\left\lfloor\frac{c}{b}\right\rfloor b^{\alpha}+\left(c-\left\lfloor\frac{c}{b}\right\rfloor b\right)^{\alpha} & \text { if } 0<b \leq c \\ 0 & \text { if } b=c=0 \\ \infty & \text { otherwise }\end{cases}
$$

Note first that (4.5) is obvious except for the first case, and hence, we will assume that $0<b \leq c$ from now on. Note also that $I^{\prime}(c, b)=\inf \left\{I(\xi): \xi \in \mathbb{D}_{\leqslant \infty}, \phi(\xi)=(c, b)\right\}$ since $I(\xi)=\infty$ if $\xi \notin \mathbb{D}_{\leqslant \infty}$. Set $\mathcal{C} \triangleq\left\{\xi \in \mathbb{D}_{\leqslant \infty},(c, b)=\phi(\xi)\right\}$ and remember that any $\xi \in \mathbb{D}_{\leqslant \infty}$ admits the following representation:

$$
\xi=\sum_{i=1}^{\infty} x_{i} \mathbb{1}_{\left[u_{i}, 1\right]},
$$

where $u_{i}$ 's are distinct in $(0,1)$ and $x_{i}$ 's are non-negative and sorted in a decreasing order. Consider a step function $\xi_{0} \in \mathcal{C}$, with $\left\lfloor\frac{c}{b}\right\rfloor$ jumps of size $b$ and one jump of size $c-\left\lfloor\frac{c}{b}\right\rfloor b$, so that $\xi_{0}=$ $\left.\sum_{i=1}^{\left\lfloor\frac{c}{b}\right\rfloor} b \mathbb{1}_{\left[u_{i}, 1\right]}+\left(c-\left\lfloor\frac{c}{b}\right\rfloor\right) \mathbb{1}_{\left[u\left\lfloor\frac{c}{b}\right\rfloor+1\right.}, 1\right]$. Clearly, $\phi\left(\xi_{0}\right)=(c, b)$ and $I\left(\xi_{0}\right)=\left\lfloor\frac{c}{b}\right\rfloor b^{\alpha}+\left(c-\left\lfloor\frac{c}{b}\right\rfloor b\right)^{\alpha}$. Since $\xi_{0} \in \mathcal{C}$, the infimum of $I$ over $\mathcal{C}$ should be at most $I\left(\xi_{0}\right)$ i.e., $I\left(\xi_{0}\right) \geq I^{\prime}(c, b)$.

To prove that $\xi_{0}$ is the minimizer of $I$ over $\mathcal{C}$, we will show that $I(\xi) \geq I\left(\xi_{0}\right)$ for any $\xi=$ $\sum_{i=1}^{\infty} x_{i} \mathbb{1}_{\left[u_{i}, 1\right]} \in \mathcal{C}$ by constructing $\xi^{\prime}$ such that $I(\xi) \geq I\left(\xi^{\prime}\right)$ while $I\left(\xi^{\prime}\right)=I\left(\xi_{0}\right)$. There has to be an integer $k$ such that $x_{k}^{\prime} \triangleq \sum_{i=k}^{\infty} x_{i} \leq b$. Let $\xi^{1} \triangleq \sum_{i=1}^{k} x_{i}^{1} \mathbb{1}_{\left[u_{i}, 1\right]}$ where $x_{i}^{1}$ is the $i^{\text {th }}$ largest element of $\left\{x_{1}, \ldots, x_{k-1}, x_{k}^{\prime}\right\}$. Then, $\xi^{1} \in \mathcal{C}$ and $I\left(\xi^{1}\right) \leq I(\xi)$ due to the sub-additivity of $x \mapsto x^{\alpha}$. Now, given $\xi^{j}=\sum_{i=1}^{k} x_{i}^{j} \mathbb{1}_{\left[u_{i}, 1\right]}$, we construct $\xi^{j+1}$ as follows. Find the first $l$ such that $x_{l}^{j}<b$. If $x_{l}^{j}=0$ or $x_{l+1}^{j}=0$, set $\xi^{j+1} \triangleq \xi^{j}$. Otherwise, find the first $m$ such that $x_{m+1}^{j}=0$ and merge the $l^{\text {th }}$ jump and the $m^{\text {th }}$ jump. More specifically, set $x_{l}^{j+1} \triangleq x_{l}^{j}+x_{m}^{j} \wedge\left(b-x_{l}^{j}\right), x_{m}^{j+1} \triangleq x_{m}^{j}-x_{m}^{j} \wedge\left(b-x_{l}^{j}\right)$, $x_{i}^{j+1} \triangleq x_{i}^{j}$ for $i \neq l, m$, and $\xi^{j+1} \triangleq \sum_{i=1}^{k} x_{i}^{j+1} \mathbb{1}_{\left[u_{i}, 1\right]}$. Note that $x_{l}^{j+1}+x_{m}^{j+1}=x_{l}^{j}+x_{m}^{j}$ while either $x_{l}^{j+1}=b$ or $x_{m}^{j+1}=0$. That is, compared to $\xi^{j}, \xi^{j+1}$ has either one less jump or one more jump with size $b$, while the total sum of the jump sizes and the maximum jump size remain the same. From this observation and the concavity of $x \mapsto x^{\alpha}$, it is straightforward to check that $I\left(\xi^{j+1}\right) \leq I\left(\xi^{j}\right)$. By iterating this procedure $k$ times, we arrive at $\xi^{\prime} \triangleq \xi^{k}$ such that all the jump sizes of $\xi^{\prime}$ are $b$, or there is only one jump whose size is not $b$. From this, we see that $\xi^{k}$ has to coincide with $\xi_{0}$. We conclude that $I(\xi) \geq I\left(\xi^{1}\right) \geq \cdots \geq I\left(\xi^{k}\right)=I\left(\xi^{\prime}\right)=I\left(\xi_{0}\right)$, proving that $\xi_{0}$ is indeed a minimizer.

Now we check that $\Psi_{I^{\prime}}(\gamma) \triangleq\left\{(c, b): I^{\prime}(c, b) \leq \gamma\right\}$ is compact for each $\gamma \in[0, \infty)$ so that $I^{\prime}$ is a good rate function. It is clear that $\Psi_{I^{\prime}}(\gamma)$ is bounded. To see that $\Psi_{I^{\prime}}(\gamma)$ is closed, suppose that $\left(c_{1}, b_{1}\right) \notin \Psi_{I^{\prime}}(\gamma)$. In case $0<b_{1}<c_{1}$, note that $I^{\prime}$ can be written as $I^{\prime}(c, b)=b^{\alpha}\left\{(c / b-\lfloor c / b\rfloor)^{\alpha}+\right.$ $\lfloor c / b\rfloor\}$, from which it is easy to see that $I^{\prime}$ is continuous at such $\left(c_{1}, b_{1}\right)$ 's. Therefore, one can find an open ball around $\left(c_{1}, b_{1}\right)$ in such a way that it doesn't intersect with $\Psi_{I^{\prime}}(\gamma)$. By considering the cases $c_{1}<b_{1}, b_{1}=0, b_{1}=c_{1}$ separately, the rest of the cases are straightforward to check. We thus conclude that $I^{\prime}$ is a good rate function. Now we can apply Corollary 3.1. Note that

$$
\inf _{(x, y) \in[c, \infty) \times[0, b]} I^{\prime}(x, y)=\inf _{(x, y) \in(c, \infty) \times[0, b)} I^{\prime}(x, y)=I^{\prime}(c, b) .
$$


That is the large deviation lower and upper bound coincide and hence,

$$
\begin{aligned}
\lim _{n \rightarrow \infty} \frac{\log \mathbf{P}\left(\sup _{t \in[0,1]} \bar{X}_{n}(t) \geq c, \sup _{t \in[0,1]}\left|\bar{X}_{n}(t)-\bar{X}_{n}(t-)\right| \leq b\right)}{L(n) n^{\alpha}} \\
\quad= \begin{cases}\left\lfloor\frac{c}{b}\right\rfloor b^{\alpha}+\left(c-\left\lfloor\frac{c}{b}\right\rfloor b\right)^{\alpha} & \text { if } 0<b \leq c, \\
0 & \text { if } b=c=0, \\
\infty & \text { otherwise. }\end{cases}
\end{aligned}
$$

From the expression of the rate function, it can be inferred that the most likely way level $c$ is reached is due to $\left\lfloor\frac{c}{b}\right\rfloor$ jumps of size $b$ and one jump of size $\left(c-\left\lfloor\frac{c}{b}\right\rfloor b\right)$. If we compare this with the insights obtained from the case of truncated regularly varying tails in Rhee, Blanchet and Zwart (2016), we see that the total number of jumps is identical, but the size of the jumps are deterministic and non-identical, while in the regularly varying case, they are identically distributed with Pareto distribution.

5. Proofs. This section provides technical proofs.

5.1. Lower semi-continuity of $I$ and $I^{d}$. Recall the definition of $I$ in (2.3) and $I^{d}$ in (2.11).

LEMMA 5.1. I and $I^{d}$ are lower semi-continuous, and hence, rate functions.

Proof. We start with $I$. To show that the sub-level sets $\Psi_{I}(\gamma)$ are closed for each $\gamma<\infty$, let $\xi$ be any given path that does not belong to $\Psi_{I}(\gamma)$. We will show that there exists an $\epsilon>0$ such that $d_{J_{1}}\left(\xi, \Psi_{I}(\gamma)\right) \geq \epsilon$. Note that $\Psi_{I}(\gamma)^{\mathrm{c}}=(A \cap B \cap C \cap D)^{\mathrm{c}}=\left(A^{\mathrm{c}}\right) \cup\left(A \cap B^{c}\right) \cup\left(A \cap B \cap C^{\mathrm{c}}\right) \cup$ $\left(A \cap B \cap C \cap D^{\mathrm{c}}\right)$ where

$$
\begin{array}{ll}
A=\{\xi \in \mathbb{D}: \xi(0)=0 \text { and } \xi(1)=\xi(1-)\}, & B=\{\xi \in \mathbb{D}: \xi \text { is non-decreasing }\}, \\
C=\{\xi \in \mathbb{D}: \xi \text { is a pure jump function }\}, & D=\left\{\xi \in \mathbb{D}: \sum_{t \in[0,1]}(\xi(t)-\xi(t-))^{\alpha} \leq \gamma\right\} .
\end{array}
$$

For $\xi \in A^{\mathrm{c}}$, we will show that $d_{J_{1}}\left(\xi, \Psi_{I}(\gamma)\right) \geq \delta$ where $\delta=\frac{1}{2} \max \{|\xi(0)|,|\xi(1)-\xi(1-)|\}$. Suppose not so that there exists $\zeta \in \Psi_{I}(\gamma)$ such that $d_{J_{1}}(\xi, \zeta)<\delta$. Then $|\zeta(0)|>|\xi(0)|-2 \delta$ and $\mid \zeta(1)-$ $\zeta(1-)|>| \xi(1)-\xi(1-) \mid-2 \delta$. That is, $\max \{|\zeta(0)|,|\zeta(1)-\zeta(1-)|\}>\max \{|\xi(0)|-2 \delta, \mid \xi(1)-$ $\xi(1-) \mid-2 \delta\}=0$. Therefore, $\zeta \in A^{c}$, and hence, $I(\zeta)=\infty$, which contradicts the assumption that $\zeta \in \Psi_{I}(\gamma)$.

If $\xi \in A \cap B^{\mathrm{c}}$, there are $T_{s}<T_{t}$ such that $c \triangleq \xi\left(T_{s}\right)-\xi\left(T_{t}\right)>0$. We claim that $d_{J_{1}}(\xi, \zeta) \geq c / 2$ if $\zeta \in \Psi_{I}(\gamma)$. Suppose that this is not the case and there exists $\zeta \in \Psi_{I}(\gamma)$ such that $d_{J_{1}}(\xi, \zeta)<c / 2$. Let $\lambda$ be a non-decreasing homeomorphism $\lambda:[0,1] \rightarrow[0,1]$ such that $\|\zeta \circ \lambda-\xi\|_{\infty}<c / 2$, in particular, $\zeta \circ \lambda\left(T_{s}\right)>\xi\left(T_{s}\right)-c / 2$ and $\zeta \circ \lambda\left(T_{t}\right)<\xi\left(T_{t}\right)+c / 2$. Subtracting the latter inequality from the former, we get $\zeta \circ \lambda\left(T_{s}\right)-\zeta \circ \lambda\left(T_{t}\right)>\xi\left(T_{s}\right)-\xi\left(T_{t}\right)-c=0$. That is, $\zeta$ is not non-decreasing, which is contradictory to the assumption $\zeta \in \Psi_{I}(\gamma)$. Therefore, the claim has to be the case.

If $\xi \in A \cap B \cap C^{\mathrm{c}}$, there exists an interval $\left[T_{s}, T_{t}\right]$ so that $\xi$ is continuous and $c \triangleq \xi\left(T_{t}\right)-\xi\left(T_{s}\right)>0$. Pick $\delta$ small enough so that $(c-2 \delta)(2 \delta)^{\alpha-1}>\gamma$. We will show that $d_{J_{1}}\left(\xi, \Psi_{I}(\gamma)\right) \geq \delta$. Suppose that $\zeta \in \Psi_{I}(\gamma)$ and $d_{J_{1}}(\zeta, \xi)<\delta$, and let $\lambda$ be a non-decreasing homeomorphism such that 
$\|\zeta \circ \lambda-\xi\|_{\infty}<\delta$. Note that this implies that each of the jump sizes of $\zeta \circ \lambda$ in $\left[T_{s}, T_{t}\right]$ has to be less than $2 \delta$. On the other hand, $\zeta \circ \lambda\left(T_{t}\right) \geq \xi\left(T_{t}\right)-\delta$ and $\zeta \circ \lambda\left(T_{s}\right) \leq \xi\left(T_{s}\right)+\delta$, which in turn implies that $\zeta \circ \lambda\left(T_{t}\right)-\zeta \circ \lambda\left(T_{s}\right) \geq c-2 \delta$. Since $\zeta \circ \lambda$ is a non-decreasing pure jump function,

$$
\begin{aligned}
c-2 \delta & \leq \zeta \circ \lambda\left(T_{t}\right)-\zeta \circ \lambda\left(T_{s}\right)=\sum_{t \in\left(T_{s}, T_{t}\right]}(\zeta \circ \lambda(t)-\zeta \circ \lambda(t-)) \\
& =\sum_{t \in\left(T_{s}, T_{t}\right]}(\zeta \circ \lambda(t)-\zeta \circ \lambda(t-))^{\alpha}(\zeta \circ \lambda(t)-\zeta \circ \lambda(t-))^{1-\alpha} \\
& \leq \sum_{t \in\left(T_{s}, T_{t}\right]}(\zeta \circ \lambda(t)-\zeta \circ \lambda(t-))^{\alpha}(2 \delta)^{1-\alpha} .
\end{aligned}
$$

That is, $\sum_{t \in\left(T_{s}, T_{t}\right]}(\zeta \circ \lambda(t)-\zeta \circ \lambda(t-))^{\alpha} \geq(2 \delta)^{\alpha-1}(c-2 \delta)>\gamma$, which is contradictory to our assumption that $\zeta \in \Psi_{I}(\gamma)$. Therefore, $d_{J_{1}}\left(\xi, \Psi_{I}(\gamma)\right) \geq \delta$.

Finally, let $\xi \in A \cap B \cap C \cap D^{\mathrm{c}}$. This implies that $\xi$ admits the following representation: $\xi=\sum_{i=1}^{\infty} x_{i} \mathbb{1}_{\left[u_{i}, 1\right]}$ where $u_{i}$ 's are all distinct in $(0,1)$ and $\sum_{i=1}^{\infty} x_{i}^{\alpha}>\gamma$. Choose $k$ large enough and $\delta$ small enough so that $\sum_{i=1}^{k}\left(x_{i}-2 \delta\right)^{\alpha}>\gamma$. We will show that $d_{J_{1}}\left(\xi, \Psi_{I}(\gamma)\right) \geq \delta$. Suppose that this is not the case. That is, there exists $\zeta \in \Psi_{I}(\gamma)$ so that $d_{J_{1}}(\xi, \zeta)<\delta$. Let $\lambda$ be a non-decreasing homeomorphism such that $\|\zeta \circ \lambda-\xi\|_{\infty}<\delta$. Thus for each $i \in\{1, \ldots, k\}, \zeta \circ \lambda\left(u_{i}\right)-\zeta \circ \lambda\left(u_{i}-\right) \geq$ $\xi\left(u_{i}\right)-\xi\left(u_{i}-\right)-2 \delta=x_{i}-2 \delta$, and hence,

$$
I(\zeta)=\sum_{t \in[0,1]}\left(\zeta \circ \lambda\left(t_{i}\right)-\zeta \circ \lambda\left(t_{i}-\right)\right)^{\alpha} \geq \sum_{i=1}^{k}\left(\zeta \circ \lambda\left(u_{i}\right)-\zeta \circ \lambda\left(u_{i}-\right)\right) \geq \sum_{i=1}^{k}\left(x_{i}-2 \delta\right)^{\alpha}>\gamma,
$$

which contradicts the assumption that $\zeta \in \Psi_{I}(\gamma)$.

$I^{d}$ is lower semi-continuous since it is a sum of lower semi-continuous functions.

\subsection{Proof of Proposition 2.1.}

Proof of Proposition 2.1. We start with the extended large deviation upper bound. For any measurable set $A$,

$$
\begin{aligned}
\mathbf{P}\left(X_{n} \in A\right) & =\mathbf{P}\left(X_{n} \in A, d\left(X_{n}, Y_{n}^{k}\right) \leq \epsilon\right)+\mathbf{P}\left(X_{n} \in A, d\left(X_{n}, Y_{n}^{k}\right)>\epsilon\right) \\
& \leq \underbrace{\mathbf{P}\left(Y_{n}^{k} \in A^{\epsilon}\right)}_{\triangleq(\mathrm{I})}+\underbrace{\mathbf{P}\left(d\left(X_{n}, Y_{n}^{k}\right)>\epsilon\right)}_{\triangleq(\mathrm{II})} .
\end{aligned}
$$

From the principle of the largest term and (i),

$$
\limsup _{n \rightarrow \infty} \frac{\log \mathbf{P}\left(X_{n} \in A\right)}{a_{n}} \leq \max \left\{-\inf _{x \in A^{2 \epsilon}} I_{k}(x), \limsup _{n \rightarrow \infty} \frac{1}{a_{n}} \log \mathbf{P}\left(d\left(X_{n}, Y_{n}^{k}\right)>\epsilon\right)\right\} .
$$

Now letting $k \rightarrow \infty$ and then $\epsilon \rightarrow 0$, (ii) and (iv) lead to

$$
\limsup _{n \rightarrow \infty} \frac{1}{a_{n}} \log \mathbf{P}\left(X_{n} \in A\right) \leq-\lim _{\epsilon \rightarrow 0} \inf _{x \in A^{\epsilon}} I(x),
$$


which is the upper bound of the extended LDP.

Turning to the lower bound, note that the lower bound is trivial if $\inf _{x \in A^{\circ}} I(x)=\infty$. Therefore, we focus on the case $\inf _{x \in A^{\circ}} I(x)<\infty$. Consider an arbitrary but fixed $\delta \in(0,1)$. In view of (iii) and (iv), one can pick $\epsilon>0$ and $k \geq 1$ in such a way that

$-\inf _{x \in A^{\circ}} I(x) \leq-\inf _{x \in A^{-\epsilon}} I_{k}(x)+\delta \quad$ and $\quad \limsup _{n \rightarrow \infty} \frac{\log \mathbf{P}\left(d\left(X_{n}, Y_{n}^{k}\right)>\epsilon\right)}{a_{n}} \leq-\inf _{x \in A^{\circ}} I(x)-1$.

Hence

$$
\limsup _{n \rightarrow \infty} \frac{\log \mathbf{P}\left(d\left(X_{n}, Y_{n}^{k}\right)>\epsilon\right)}{a_{n}} \leq-\inf _{x \in A^{-\epsilon}} I_{k}(x)+\delta-1
$$

Now, from (5.3) and the lower bound of the assumed extended LDP for $Y_{n}^{k}$, one can easily verify that

$$
\frac{\mathbf{P}\left(d\left(X_{n}, Y_{n}^{k}\right)>\epsilon\right)}{\mathbf{P}\left(Y_{n}^{k} \in A^{-\epsilon}\right)} \rightarrow 0
$$

as $n \rightarrow \infty$. Using (5.4) and the first inequality of (5.2),

$$
\begin{aligned}
\liminf _{n \rightarrow \infty} \frac{1}{a_{n}} \log \mathbf{P}\left(X_{n} \in A\right) & \geq \liminf _{n \rightarrow \infty} \frac{1}{a_{n}} \log \mathbf{P}\left(Y_{n}^{k} \in A^{-\epsilon}, d\left(X_{n}, Y_{n}^{k}\right) \leq \epsilon\right) \\
& \geq \liminf _{n \rightarrow \infty} \frac{1}{a_{n}} \log \left(\mathbf{P}\left(Y_{n}^{k} \in A^{-\epsilon}\right)-\mathbf{P}\left(d\left(X_{n}, Y_{n}^{k}\right)>\epsilon\right)\right) \\
& =\liminf _{n \rightarrow \infty} \frac{1}{a_{n}} \log \left(\mathbf{P}\left(Y_{n}^{k} \in A^{-\epsilon}\right)\left(1-\frac{\mathbf{P}\left(d\left(X_{n}, Y_{n}^{k}\right)>\epsilon\right)}{\mathbf{P}\left(Y_{n}^{k} \in A^{-\epsilon}\right)}\right)\right) \\
& =\liminf _{n \rightarrow \infty} \frac{1}{a_{n}} \log \mathbf{P}\left(Y_{n}^{k} \in A^{-\epsilon}\right) \geq-\inf _{x \in A^{-\epsilon}} I_{k}(x) \geq-\inf _{x \in A} I(x)-\delta .
\end{aligned}
$$

Since $\delta$ was arbitrary in $(0,1)$, the lower bound is proved by letting $\delta \rightarrow 0$.

5.3. Proof of Lemma 2.1. We prove Lemma 2.1 in several steps. Before we proceed, we introduce some notation and a distributional representation of the compound Poisson processes $Y_{n}$. The following representation for the time-scaled compound Poisson process is a straightforward modification of the distributional representation on page 305 of Lindskog, Resnick and Roy (2014); see also exercise 5.4 on page 163 of Resnick (2007):

$$
\int_{x \geq 1} x N([0, n \cdot] \times d x) \stackrel{\mathcal{D}}{=} \sum_{l=1}^{\tilde{N}_{n}} Q_{n}^{\leftarrow}\left(\Gamma_{l}\right) \mathbb{1}_{\left[U_{l}, 1\right]}(\cdot)
$$

where $\Gamma_{l}=E_{1}+E_{2}+\ldots+E_{l} ; E_{i}$ 's are i.i.d. and standard exponential random variables; $U_{l}$ 's are i.i.d. and uniform variables in $[0,1] ; \tilde{N}_{n}=N_{n}([0,1] \times[1, \infty)) ; N_{n}=\sum_{l=1}^{\infty} \delta_{\left(U_{l}, Q_{n}^{\leftarrow}\left(\Gamma_{l}\right)\right)}$, where $\delta_{(x, y)}$ is the Dirac measure concentrated on $(x, y) ; Q_{n}(x) \triangleq n \nu[x, \infty)$, and $Q_{n}^{\leftarrow}(y) \triangleq \inf \{s>0: n \nu[s, \infty)<y\}$. 
It should be noted that $\tilde{N}_{n}$ is the largest $l$ such that $\Gamma_{l} \leq n \nu_{1}$, where $\nu_{1} \triangleq \nu[1, \infty)$, and hence, $\tilde{N}_{n} \sim \operatorname{Poisson}\left(n \nu_{1}\right)$. Recall the definition of $\bar{J}_{n}^{k}$ - the process which keeps (up to) the $k$ biggest $Z_{i}$ 's among $Z_{1}, \ldots, Z_{N(n)}$. From this and the observation that $Q_{n}^{\leftarrow}\left(\Gamma_{l}\right)$ is decreasing in $l$, we conclude that $\bar{J}_{n}^{k}$ has the following distributional representation:

$$
\bar{J}_{n}^{k}(\cdot) \stackrel{\mathcal{D}}{=} \underbrace{\frac{1}{n} \sum_{i=1}^{k} Q_{n}^{\leftarrow}\left(\Gamma_{i}\right) \mathbb{1}_{\left[U_{i}, 1\right]}(\cdot)}_{\triangleq \hat{J}_{n}^{\leqslant k}(\cdot)}-\underbrace{\frac{1}{n} \mathbb{1}\left\{\tilde{N}_{n}<k\right\} \sum_{i=\tilde{N}_{n}+1}^{k} Q_{n}^{\leftarrow}\left(\Gamma_{i}\right) \mathbb{1}_{\left[U_{i}, 1\right]}(\cdot)}_{\triangleq \tilde{J}_{n}^{\leqslant k}(\cdot)} .
$$

Roughly speaking, $\left(Q_{n}^{\leftarrow}\left(\Gamma_{1}\right) / n, \ldots, Q_{n}^{\leftarrow}\left(\Gamma_{k}\right) / n\right)$ represents the $k$ largest jump sizes of $\bar{Y}_{n}$, and $\hat{J}_{n}^{\leqslant k}$ can be regarded as the process obtained by keeping only $k$ largest jumps of $\bar{Y}_{n}$ while disregarding the rest. Lemma 5.2 and Corollary 5.1 prove an LDP for $\left(Q_{n}^{\leftarrow}\left(\Gamma_{1}\right) / n, \ldots, Q_{n}^{\leftarrow}\left(\Gamma_{k}\right) / n, U_{1}, \ldots, U_{k}\right)$ Consequently, Lemma 5.3 yields a sample path LDP for $\hat{J}_{n}^{\leqslant k}$. Finally, Lemma 2.1 is proved by showing that $\bar{J}_{n}^{k}$ satisfies the same LDP as the one satisfied by $\hat{J}_{n}^{\leqslant k}$.

LEMMA 5.2. $\quad\left(Q_{n}^{\leftarrow}\left(\Gamma_{1}\right) / n, Q_{n}^{\leftarrow}\left(\Gamma_{2}\right) / n, \ldots, Q_{n}^{\leftarrow}\left(\Gamma_{k}\right) / n\right)$ satisfies a large deviation principle in $\mathbb{R}_{+}^{k}$ with normalization $L(n) n^{\alpha}$, and with good rate function

$$
\check{I}_{k}\left(x_{1}, \ldots x_{k}\right)=\left\{\begin{array}{ll}
\sum_{i=1}^{k} x_{i}^{\alpha} & \text { if } x_{1} \geq x_{2} \geq \cdots \geq x_{k} \geq 0 \\
\infty, & \text { o.w. }
\end{array} .\right.
$$

Proof. It is straightforward to check that $\check{I}_{k}$ is a good rate function. For each $f \in \mathcal{C}_{b}\left(\mathbb{R}_{+}^{k}\right)$, let

$$
\Lambda_{f}^{*} \triangleq \lim _{n \rightarrow \infty} \frac{1}{L(n) n^{\alpha}} \log \left(\mathbf{E} e^{L(n) n^{\alpha} f\left(Q_{n}^{\leftarrow}\left(\Gamma_{1}\right) / n, Q_{n}^{\leftarrow}\left(\Gamma_{2}\right) / n, \ldots, Q_{n}^{\overleftarrow{n}}\left(\Gamma_{k}\right) / n\right)}\right)
$$

Applying Bryc's inverse Varadhan lemma (see e.g. Theorem 4.4.13 of Dembo and Zeitouni, 2010), we can show that $\left(Q_{n}^{\leftarrow}\left(\Gamma_{1}\right) / n, \ldots, Q_{n}^{\leftarrow}\left(\Gamma_{k}\right) / n\right)$ satisfies a large deviation principle with speed $L(n) n^{\alpha}$ and good rate function $\check{I}_{k}(x)$ if

$$
\Lambda_{f}^{*}=\sup _{x \in \mathbb{R}_{+}^{k}}\left\{f(x)-\check{I}_{k}(x)\right\}
$$

for every $f \in \mathcal{C}_{b}\left(\mathbb{R}_{+}^{k}\right)$.

To prove (5.7), fix $f \in C_{b}\left(\mathbb{R}_{+}^{k}\right)$ and let $M$ be a constant such that $|f(x)| \leq M$ for all $x \in \mathbb{R}_{+}^{k}$. We first claim that the supremum of $\Lambda_{f} \triangleq f-\check{I}_{k}$ is finite and attained. Pick a constant $R$ so that $R^{\alpha}>2 M$. Since $\Lambda_{f}$ is upper semi-continuous on $[0, R]^{k}$, which is compact, there exists a maximizer $\hat{x} \triangleq\left(\hat{x}_{1}, \ldots, \hat{x}_{k}\right)$ of $\Lambda_{f}$ on $[0, R]^{k}$. Since

$$
\sup _{x \in[0, R]^{k}}\left\{f(x)-\check{I}_{k}(x)\right\} \geq \sup _{x \in[0, R]^{k}} f(x) \geq-M
$$

and

$$
\sup _{x \in \mathbb{R}_{+}^{k} \backslash[0, R]^{k}}\left\{f(x)-\check{I}_{k}(x)\right\}<\sup _{x \in \mathbb{R}_{+}^{k} \backslash[0, R]^{k}}\{f(x)-2 M\} \leq-M,
$$


$\hat{x}$ is, in fact, a global maximizer. Now, it is enough to prove that

$$
\Lambda_{f}(\hat{x}) \leq \liminf _{n \rightarrow \infty} \frac{1}{L(n) n^{\alpha}} \log \Upsilon_{f}(n) \quad \text { and } \quad \limsup _{n \rightarrow \infty} \frac{1}{L(n) n^{\alpha}} \log \Upsilon_{f}(n) \leq \Lambda_{f}(\hat{x}),
$$

where

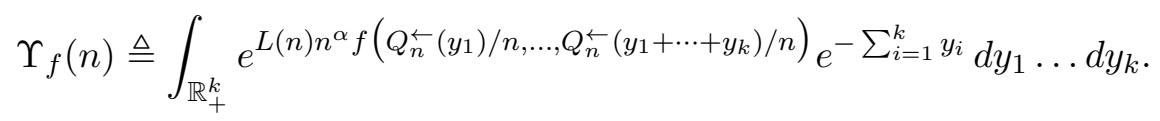

We start with the lower bound-i.e., the first inequality of (5.8). Fix an arbitrary $\epsilon>0$. Since $\Lambda_{f}$ is continuous on $A_{\infty} \triangleq\left\{\left(x_{1}, \ldots, x_{k}\right) \in \mathbb{R}_{+}^{k}: x_{1} \geq \cdots \geq x_{k}\right\}$, there exists $\delta>0$ such that $x \in B(\hat{x} ; 2 \sqrt{k} \delta) \cap A_{\infty}$ implies $\Lambda_{f}(x) \geq \Lambda_{f}(\hat{x})-\epsilon$. Since $\prod_{j=1}^{k}\left[\hat{x}_{j}+\delta, \hat{x}_{j}+2 \delta\right] \subseteq B(\hat{x} ; 2 \sqrt{k} \delta)$ and $Q_{n}^{\leftarrow}(\cdot)$ is non-increasing, $Q_{n}^{\leftarrow}\left(\sum_{i=1}^{j} y_{i}\right) / n \in\left[\hat{x}_{j}+\delta, \hat{x}_{j}+2 \delta\right]$ for all $j=1, \ldots, k$ implies $\left(Q_{n}^{\leftarrow}\left(y_{1}\right) / n, \ldots, Q_{n}^{\leftarrow}\left(y_{1}+\cdots+y_{k}\right) / n\right) \in B(\hat{x} ; 2 \sqrt{k} \delta)$, and hence,

$$
\Lambda_{f}\left(Q_{n}^{\leftarrow}\left(y_{1}\right) / n, \ldots, Q_{n}^{\leftarrow}\left(y_{1}+\cdots+y_{k}\right) / n\right) \geq \Lambda_{f}(\hat{x})-\epsilon
$$

That is, if we define $D_{n}^{j}\left(=D_{n}^{y_{1}, \ldots, y_{j-1}}\right)$ as

$$
D_{n}^{j} \triangleq\left\{y_{j} \in \mathbb{R}_{+}: Q_{n}^{\leftarrow}\left(\sum_{i=1}^{j} y_{i}\right) / n \in\left[\hat{x}_{j}+\delta, \hat{x}_{j}+2 \delta\right]\right\}
$$

then (5.9) holds for $\left(y_{1}, \ldots, y_{k}\right)$ 's such that $y_{j} \in D_{n}^{j}$ for $j=1, \ldots, k$. Therefore,

$$
\begin{aligned}
& \Upsilon_{f}(n)=\int_{\mathbb{R}_{+}^{k}} e^{L(n) n^{\alpha} \Lambda_{f}\left(Q_{n}^{\leftarrow}\left(y_{1}\right) / n, \ldots, Q_{n}^{\leftarrow}\left(y_{1}+\cdots+y_{k}\right) / n\right)+L(n) \sum_{i=1}^{k} Q_{n}^{\leftarrow}\left(\sum_{j=1}^{i} y_{j}\right)^{\alpha}-\sum_{i=1}^{k} y_{i}} d y_{1} \ldots d y_{k} \\
& \geq \int_{D_{n}^{1}} \ldots \int_{D_{n}^{k}} e^{L(n) n^{\alpha} \Lambda_{f}\left(Q_{n}^{\leftarrow}\left(y_{1}\right) / n, \ldots, Q_{n}^{\leftarrow}\left(y_{1}+\cdots+y_{k}\right) / n\right)+L(n) \sum_{i=1}^{k} Q_{n}^{\leftarrow}\left(\sum_{j=1}^{i} y_{j}\right)^{\alpha}-\sum_{i=1}^{k} y_{i}} d y_{k} \ldots d y_{1} \\
& \geq \int_{D_{n}^{1}} \ldots \int_{D_{n}^{k}} e^{L(n) n^{\alpha}\left(\Lambda_{f}\left(\hat{x}_{1}, \ldots, \hat{x}_{k}\right)-\epsilon\right)} e^{L(n) \sum_{i=1}^{k} Q_{n}^{\leftarrow}\left(\sum_{j=1}^{i} y_{j}\right)^{\alpha}-\sum_{i=1}^{k} y_{i}} d y_{k} \ldots d y_{1} \\
& \geq \int_{D_{n}^{1}} \ldots \int_{D_{n}^{k}} e^{L(n) n^{\alpha}\left(\Lambda_{f}\left(\hat{x}_{1}, \ldots, \hat{x}_{k}\right)-\epsilon\right)} e^{L(n) \sum_{i=1}^{k}\left(n\left(\hat{x}_{i}+\delta\right)\right)^{\alpha}-\sum_{i=1}^{k} y_{i}} d y_{k} \ldots d y_{1} \\
& =\underbrace{e^{L(n) n^{\alpha}\left(\Lambda_{f}\left(\hat{x}_{1}, \ldots, \hat{x}_{k}\right)-\epsilon\right)}}_{\triangleq(\mathrm{I})_{n}} \underbrace{e^{L(n) \sum_{i=1}^{k}\left(n\left(\hat{x}_{i}+\delta\right)\right)^{\alpha}}}_{\triangleq(\mathrm{II})_{n}} \underbrace{\int_{D_{n}^{1}} \ldots \int_{D_{n}^{k}} e^{-\sum_{i=1}^{k} y_{i}} d y_{k} \ldots d y_{1}}_{\triangleq(\mathrm{III})_{n}},
\end{aligned}
$$

where the first equality is obtained by adding and subtracting $L(n) \sum_{i=1}^{k} Q_{n}^{\leftarrow}\left(\sum_{j=1}^{i} y_{j}\right)^{\alpha}$ to the exponent of the integrand. Note that by the construction of $D_{n}^{j}$ 's,

$$
Q_{n}\left(n\left(\hat{x}_{j}+2 \delta\right)\right) \leq y_{1}+\cdots+y_{j} \leq Q_{n}\left(n\left(\hat{x}_{j}+\delta\right)\right)
$$

on the domain of the integral in (III $)_{n}$, and hence,

$$
(\mathrm{III})_{n} \geq e^{-Q_{n}\left(n\left(\hat{x}_{k}+\delta\right)\right)} \prod_{i=1}^{k}\left(Q_{n}\left(n\left(\hat{x}_{i}+\delta\right)\right)-Q_{n}\left(n\left(\hat{x}_{i}+2 \delta\right)\right)\right) .
$$


Since $Q_{n}\left(n\left(\hat{x}_{k}+\delta\right)\right) \rightarrow 0$ and $L\left(n\left(\hat{x}_{i}+\delta\right)\right) n^{\alpha}\left(\hat{x}_{i}+\delta\right)^{\alpha}-L\left(n\left(\hat{x}_{i}+2 \delta\right)\right) n^{\alpha}\left(\hat{x}_{i}+2 \delta\right)^{\alpha} \rightarrow-\infty$ for each $i$,

$$
\begin{aligned}
& \liminf _{n \rightarrow \infty} \frac{1}{L(n) n^{\alpha}} \log (\mathrm{III})_{n} \\
& \geq \liminf _{n \rightarrow \infty} \frac{1}{L(n) n^{\alpha}}\left(-Q_{n}\left(n\left(\hat{x}_{k}+\delta\right)\right)\right)+\sum_{i=1}^{k} \liminf _{n \rightarrow \infty} \frac{1}{L(n) n^{\alpha}} \log \left(Q_{n}\left(n\left(\hat{x}_{i}+\delta\right)\right)-Q_{n}\left(n\left(\hat{x}_{i}+2 \delta\right)\right)\right) \\
& =\sum_{i=1}^{k} \liminf _{n \rightarrow \infty} \frac{1}{L(n) n^{\alpha}} \log \left(n e^{-L\left(n\left(\hat{x}_{i}+\delta\right)\right) n^{\alpha}\left(\hat{x}_{i}+\delta\right)^{\alpha}}\left(1-e^{L\left(n\left(\hat{x}_{i}+\delta\right)\right) n^{\alpha}\left(\hat{x}_{i}+\delta\right)^{\alpha}-L\left(n\left(\hat{x}_{i}+2 \delta\right)\right) n^{\alpha}\left(\hat{x}_{i}+2 \delta\right)^{\alpha}}\right)\right) \\
& =\sum_{i=1}^{k} \liminf _{n \rightarrow \infty}\left(\frac{-L\left(n\left(\hat{x}_{i}+\delta\right)\right) n^{\alpha}\left(\hat{x}_{i}+\delta\right)^{\alpha}}{L(n) n^{\alpha}}+\frac{\log \left(1-e^{L\left(n\left(\hat{x}_{i}+\delta\right)\right) n^{\alpha}\left(\hat{x}_{i}+\delta\right)^{\alpha}-L\left(n\left(\hat{x}_{i}+2 \delta\right)\right) n^{\alpha}\left(\hat{x}_{i}+2 \delta\right)^{\alpha}}\right)}{L(n) n^{\alpha}}\right) \\
& (5.12) \\
& =-\sum_{i=1}^{k}\left(\hat{x}_{i}+\delta\right)^{\alpha} .
\end{aligned}
$$

From this along with

$$
\liminf _{n \rightarrow \infty} \frac{1}{n^{\alpha} L(n)} \log (\mathrm{I})_{n}=\liminf _{n \rightarrow \infty} \frac{1}{n^{\alpha} L(n)} \log \left(e^{n^{\alpha} L(n)\left(\Lambda_{f}\left(\hat{x}_{1}, \ldots, \hat{x}_{k}\right)-\epsilon\right)}\right)=\Lambda_{f}\left(\hat{x}_{1}, \ldots, \hat{x}_{k}\right)-\epsilon
$$

and

$$
\liminf _{n \rightarrow \infty} \frac{1}{n^{\alpha} L(n)} \log (\mathrm{II})_{n}=\liminf _{n \rightarrow \infty} \frac{1}{n^{\alpha} L(n)} \log \left(e^{L(n) \sum_{i=1}^{k}\left(n\left(\hat{x}_{i}+\delta\right)\right)^{\alpha}}\right)=\sum_{i=1}^{k}\left(\hat{x}_{i}+\delta\right)^{\alpha}
$$

we arrive at

$$
\Lambda_{f}(\hat{x})-\epsilon \leq \liminf _{n \rightarrow \infty} \frac{1}{L(n) n^{\alpha}} \log \Upsilon_{f}(n) .
$$

Letting $\epsilon \rightarrow 0$, we obtain the lower bound of (5.8).

Turning to the upper bound, consider

$$
D_{R, n} \triangleq\left\{\left(y_{1}, y_{2}, \ldots, y_{k}\right): Q_{n}^{\leftarrow}\left(y_{1}\right) / n \leq R\right\},
$$

and decompose $\Upsilon_{f}(n)$ into two parts:

$$
\begin{aligned}
\Upsilon_{f}(n)= & \int_{D_{R, n}} e^{L(n) n^{\alpha} f\left(Q_{n}^{\leftarrow}\left(x_{1}\right) / n, \ldots, Q_{n}^{\leftarrow}\left(x_{1}+\cdots+x_{k}\right) / n\right)} e^{-\sum_{i=1}^{k} x_{i}} d x_{1} \ldots d x_{k} \\
& \quad+\int_{D_{R, n}^{c}} e^{L(n) n^{\alpha} f\left(Q_{n}^{\leftarrow}\left(x_{1}\right) / n, \ldots, Q_{n}^{\leftarrow}\left(x_{1}+\cdots+x_{k}\right) / n\right)} e^{-\sum_{i=1}^{k} x_{i}} d x_{1} \ldots d x_{k}
\end{aligned}
$$


We first evaluate the integral over $D_{R, n}^{c}$. Since $|f| \leq M$,

$$
\begin{aligned}
& \int_{D_{R, n}^{c}} e^{L(n) n^{\alpha} f\left(Q_{n}^{\leftarrow}\left(x_{1}\right) / n, \ldots, Q_{n}^{\leftarrow}\left(x_{1}+\cdots+x_{k}\right) / n\right)} e^{-\sum_{i=1}^{k} x_{i}} d x_{1} \ldots d x_{k} \\
& =\int e^{L(n) n^{\alpha} f\left(Q_{n}^{\leftarrow}\left(x_{1}\right) / n, \ldots, Q_{n}^{\leftarrow}\left(x_{1}+\cdots+x_{k}\right) / n\right)} e^{-\sum_{i=1}^{k} x_{i}} \mathbb{1}_{\left\{Q_{n}^{\leftarrow}\left(x_{1}\right) / n>R\right\}} d x_{1} \ldots d x_{k} \\
& =\int e^{L(n) n^{\alpha} f\left(Q_{n}^{\leftarrow}\left(x_{1}\right) / n, \ldots, Q_{n}^{\leftarrow}\left(x_{1}+\cdots+x_{k}\right) / n\right)} e^{-\sum_{i=1}^{k} x_{i}} \mathbb{1}_{\left\{x_{1} \leq Q_{n}(n R)\right\}} d x_{1} \ldots d x_{k} \\
& \leq \int e^{L(n) n^{\alpha} M} e^{-\sum_{i=1}^{k} x_{i}} \mathbb{1}_{\left\{x_{1} \leq Q_{n}(n R)\right\}} d x_{1} \ldots d x_{k} \leq e^{L(n) n^{\alpha} M}\left(1-e^{-Q_{n}(n R)}\right) \\
& \leq e^{L(n) n^{\alpha} M} Q_{n}(n R) .
\end{aligned}
$$

Turning to the integral over $D_{R, n}$, fix $\epsilon>0$ and pick $\left\{\check{x}^{(1)}, \ldots, \check{x}^{(m)}\right\} \subset \mathbb{R}_{+}^{k}$ in such a way that $\left\{\prod_{j=1}^{k}\left[\check{x}_{j}^{(l)}-\epsilon, \check{x}_{j}^{(l)}+\epsilon\right]\right\}_{l=1, \ldots, m}$ covers $A_{R}$. Set

$H_{R, n, l} \triangleq\left\{\left(y_{1}, \ldots, y_{k}\right) \in \mathbb{R}_{+}^{k}: Q_{n}^{\leftarrow}\left(y_{1}\right) / n \in\left[\check{x}_{1}^{(l)}-\epsilon, \check{x}_{1}^{(l)}+\epsilon\right], \ldots, Q_{n}^{\leftarrow}\left(y_{1}+\ldots+y_{k}\right) / n \in\left[\check{x}_{k}^{(l)}-\epsilon, \check{x}_{k}^{(l)}+\epsilon\right]\right\}$

Then $D_{R, n} \subseteq \bigcup_{l=1}^{m} H_{R, n, l}$, and hence,

$$
\begin{aligned}
& \int_{D_{R, n}} e^{L(n) n^{\alpha} f\left(Q_{n}^{\leftarrow}\left(y_{1}\right) / n, \ldots, Q_{n}^{\leftarrow}\left(y_{1}+\cdots+y_{k}\right) / n\right)} e^{-\sum_{i=1}^{k} y_{i}} d y_{1} \cdots d y_{k} \\
& \leq \sum_{l=1}^{m} \int_{H_{R, n, l}} e^{L(n) n^{\alpha} f\left(Q_{n}^{\leftarrow}\left(y_{1}\right) / n, \ldots, Q_{n}^{\leftarrow}\left(y_{1}+\ldots+y_{k}\right) / n\right)} e^{-\sum_{i=1}^{k} y_{i}} d y_{1} \cdots d y_{k}
\end{aligned}
$$

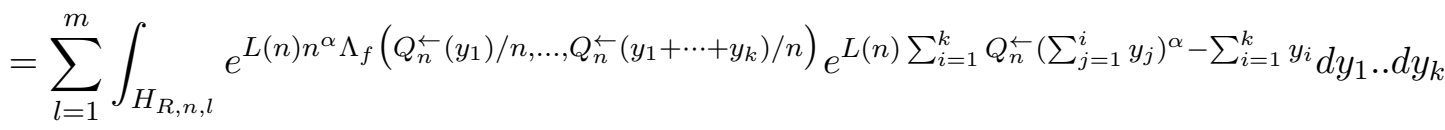

$$
\begin{aligned}
& \leq \sum_{l=1}^{m} \int_{H_{R, n, l}} e^{L(n) n^{\alpha} \Lambda_{f}\left(\hat{x}_{1}, \hat{x}_{2}, \ldots, \hat{x}_{k}\right)} e^{L(n) \sum_{i=1}^{k} Q_{n}^{\leftarrow}\left(\sum_{j=1}^{i} y_{j}\right)^{\alpha}-\sum_{i=1}^{k} y_{i}} d y_{1} \cdots d y_{k} \\
& =\sum_{l=1}^{m} \underbrace{e^{L(n) n^{\alpha} \Lambda_{f}\left(\hat{x}_{1}, \hat{x}_{2}, \ldots \hat{x}_{k}\right)} \int_{H_{R, n, l}} e^{L(n) \sum_{i=1}^{k} Q_{n}^{\leftarrow}\left(\sum_{j=1}^{i} y_{j}\right)^{\alpha}-\sum_{i=1}^{k} y_{i}} d y_{1} \cdots d y_{k}}_{\triangleq \mathrm{H}(R, n, l)},
\end{aligned}
$$

where the first equality is obtained by adding and subtracting $L(n) \sum_{i=1}^{k} Q_{n}^{\leftarrow}\left(\sum_{j=1}^{i} y_{j}\right)^{\alpha}$ to the exponent of the integrand. Since

$$
Q_{n}^{\leftarrow}\left(\sum_{j=1}^{i} y_{j}\right) / n \in\left[\check{x}_{i}^{(l)}-\epsilon, \check{x}_{i}^{(l)}+\epsilon\right] \Longrightarrow Q_{n}\left(n\left(\check{x}_{i}^{(l)}+\epsilon\right)\right) \leq \sum_{j=1}^{i} y_{j} \leq Q_{n}\left(n\left(\check{x}_{i}^{(l)}-\epsilon\right)\right)
$$


we can bound the integral in (5.15) as follows:

$$
\begin{aligned}
& \int_{H_{R, n, l}} e^{L(n) \sum_{i=1}^{k} Q_{n}^{\leftarrow}\left(\sum_{j=1}^{i} y_{j}\right)^{\alpha}-\sum_{i=1}^{k} y_{i}} d y_{1} \ldots, d y_{k} \\
& \leq \int_{H_{R, n, l}} e^{L(n) \sum_{i=1}^{k}\left(n\left(\check{x}_{i}^{(l)}-\epsilon\right)\right)^{\alpha}-\sum_{i=1}^{k} y_{i}} d y_{1} \ldots d y_{k} \\
& \leq \int_{H_{R, n, l}} e^{L(n) \sum_{i=1}^{k}\left(n\left(\check{x}_{i}^{(l)}-\epsilon\right)\right)^{\alpha}-Q_{n}\left(n\left(\check{x}_{k}^{(l)}+\epsilon\right)\right)} d y_{1} \ldots d y_{k} \\
& =e^{L(n) \sum_{i=1}^{k}\left(n\left(\check{x}_{i}^{(l)}-\epsilon\right)\right)^{\alpha}-Q_{n}\left(n\left(\check{x}_{k}^{(l)}+\epsilon\right)\right)} \int_{H_{R, n, l}} d y_{1} \ldots d y_{k} \\
& =e^{L(n) n^{\alpha} \sum_{i=1}^{k}\left(\check{x}_{i}^{(l)}-\epsilon\right)^{\alpha}-Q_{n}\left(n\left(\check{x}_{k}^{(l)}+\epsilon\right)\right)} \prod_{i=1}^{k}\left(Q_{n}\left(n\left(\check{x}_{i}^{(l)}-\epsilon\right)\right)-Q_{n}\left(n\left(\check{x}_{i}^{(l)}+\epsilon\right)\right)\right) .
\end{aligned}
$$

With (5.15) and (5.16), a straightforward calculation as in the lower bound leads to

$$
\begin{aligned}
& \limsup _{n \rightarrow \infty} \frac{1}{L(n) n^{\alpha}} \log \mathrm{H}(R, n, l) \\
& \leq \limsup _{n \rightarrow \infty} \frac{1}{L(n) n^{\alpha}} \log \left(e^{L(n) n^{\alpha} \Lambda_{f}\left(\hat{x}_{1}, \hat{x}_{2}, \ldots \hat{x}_{k}\right)}\right)+\limsup _{n \rightarrow \infty} \frac{1}{L(n) n^{\alpha}} \log \left(e^{L(n) n^{\alpha} \sum_{i=1}^{k}\left(\hat{x}_{i}^{(l)}-\epsilon\right)^{\alpha}-Q_{n}\left(n\left(\hat{x}_{k}^{(l)}+\epsilon\right)\right)}\right) \\
& \quad+\sum_{i=1}^{k} \limsup _{n \rightarrow \infty} \frac{1}{L(n) n^{\alpha}} \log \left(Q_{n}\left(n\left(\hat{x}_{i}^{(l)}-\epsilon\right)\right)-Q_{n}\left(n\left(\hat{x}_{i}^{(l)}+\epsilon\right)\right)\right) \\
& =\Lambda_{f}\left(\hat{x}_{1}, \ldots, \hat{x}_{k}\right) .
\end{aligned}
$$

Therefore,

$$
\begin{aligned}
& \limsup _{n \rightarrow \infty} \frac{1}{L(n) n^{\alpha}} \log \Upsilon_{f}(n) \\
& =\limsup _{n \rightarrow \infty} \frac{1}{L(n) n^{\alpha}} \log \left(e^{L(n) n^{\alpha} M} Q_{n}(n R)\right) \vee \max \left\{\limsup _{n \rightarrow \infty} \frac{1}{L(n) n^{\alpha}} \log \mathrm{H}(R, n, l)\right\} \\
& \leq\left(M-R^{\alpha}\right) \vee \Lambda_{f}\left(\hat{x}_{1}, \ldots, \hat{x}_{k}\right)=\left(M-R^{\alpha}\right) \vee \sup _{x \in \mathbb{R}_{+}^{k}}\left\{f(x)-\check{I}_{k}(x)\right\} .
\end{aligned}
$$

Since $R$ was arbitrary, we can send $R \rightarrow \infty$ to arrive at the desired upper bound of (5.8).

The following corollary is immediate from Lemma 5.2 and Theorem 4.14 of Ganesh, O'Connell and Wischik (2004).

Corollary 5.1. $\left(Q_{n}^{\leftarrow}\left(\Gamma_{1}\right) / n, \ldots, Q_{n}^{\leftarrow}\left(\Gamma_{k}\right) / n, U_{1}, \ldots, U_{k}\right)$ satisfies a large deviation principle in $\mathbb{R}_{+}^{k} \times[0,1]^{k}$ with speed $L(n) n^{\alpha}$ and the good rate function

$$
\hat{I}_{k}\left(x_{1}, \ldots, x_{k}, u_{1}, \ldots, u_{k}\right) \triangleq \begin{cases}\sum_{i=1}^{k} x_{i}^{\alpha} & \text { if } x_{1} \geq x_{2} \geq \cdots \geq x_{k} \quad \text { and } u_{1}, \ldots, u_{k} \in[0,1] \\ \infty, & \text { otherwise }\end{cases}
$$


Recall that $\hat{J}_{n}^{\leqslant k}=\frac{1}{n} \sum_{i=1}^{k} Q_{n}^{\leftarrow}\left(\Gamma_{i}\right) \mathbb{1}_{\left[U_{i}, 1\right]}$ and the rate function $I_{k}$ defined in (2.6). We next prove a sample path LDP for $\hat{J}_{n}^{\leqslant k}$.

LEMMA 5.3. $\quad \hat{J}_{n}^{\leqslant k}$ satisfies the LDP in $\left(\mathbb{D}, \mathcal{T}_{J_{1}}\right)$ with speed $L(n) n^{\alpha}$ and rate function $I_{k}$.

Proof. First, we note that $I_{k}$ is indeed a rate function since the sublevel sets of $I_{k}$ equal the intersection between the sublevel sets of $I$ and a closed set $\mathbb{D}_{\leqslant k}$, and $I$ is a rate function (Lemma 5.1).

Next, we prove the LDP in $\mathbb{D}_{\leqslant k}$ w.r.t. the relative topology induced by $\mathcal{T}_{J_{1}}$. (Note that $I_{k}$ is a rate function in $\mathbb{D}_{\leqslant k}$ as well.) Set $T_{k}(x, u) \triangleq \sum_{i=1}^{k} x_{i} \mathbb{1}_{\left[u_{i}, 1\right]}$. Since

$$
\inf _{(x, u) \in T_{k}^{-1}(\xi)} \hat{I}_{k}(x, u)=I_{k}(\xi)
$$

for $\xi \in \mathbb{D}_{\leqslant k}$, the LDP in $\mathbb{D}_{\leqslant k}$ is established once we show that for any closed set $F \subseteq \mathbb{D}_{\leqslant k}$,

$$
\limsup _{n \rightarrow \infty} \frac{1}{L(n) n^{\alpha}} \log \mathbf{P}\left(\hat{J}_{n}^{\leqslant k} \in F\right) \leq-\inf _{(x, u) \in T_{k}^{-1}(F)} \hat{I}_{k}(x, u),
$$

and for any open set $G \subseteq \mathbb{D}_{\leqslant k}$,

$$
-\inf _{(x, u) \in T_{k}^{-1}(G)} \hat{I}_{k}(x, u) \leq \liminf _{n \rightarrow \infty} \frac{1}{L(n) n^{\alpha}} \log \mathbf{P}\left(\hat{J}_{n}^{\leqslant k} \in G\right) .
$$

We start with the upper bound. Note that

$$
\begin{aligned}
& \limsup _{n \rightarrow \infty} \frac{1}{L(n) n^{\alpha}} \log \mathbf{P}\left(\hat{J}_{n}^{\leqslant k} \in F\right) \\
& =\limsup _{n \rightarrow \infty} \frac{1}{L(n) n^{\alpha}} \log \mathbf{P}\left(\left(Q_{n}^{\leftarrow}\left(\Gamma_{1}\right), \ldots, Q_{n}^{\leftarrow}\left(\Gamma_{k}\right), U_{1}, \ldots, U_{k}\right) \in T_{k}^{-1}(F)\right) \\
& \leq \limsup _{n \rightarrow \infty} \frac{1}{L(n) n^{\alpha}} \log \mathbf{P}\left(\left(Q_{n}^{\leftarrow}\left(\Gamma_{1}\right), \ldots, Q_{n}^{\leftarrow}\left(\Gamma_{k}\right), U_{1}, \ldots, U_{k}\right) \in T_{k}^{-1}(F)^{-}\right) \\
& \leq-\inf _{\left(x_{1}, \ldots, x_{k}, u_{1}, \ldots, u_{k}\right) \in T_{k}^{-1}(F)^{-}} \hat{I}_{k}\left(x_{1}, \ldots, x_{k}, u_{1}, \ldots, u_{k}\right) .
\end{aligned}
$$

In view of (5.18), it is therefore enough for the upper bound to show that

$$
\inf _{(x, u) \in T_{k}^{-1}(F)} \hat{I}_{k}(x, u) \leq \inf _{(x, u) \in T_{k}^{-1}(F)^{-}} \hat{I}_{k}(x, u) .
$$

To prove this, we proceed with proof by contradiction. Suppose that

$$
c \triangleq \inf _{(x, u) \in T_{k}^{-1}(F)} \hat{I}_{k}(x, u)>\inf _{(x, u) \in T_{k}^{-1}(F)^{-}} \hat{I}_{k}(x, u) .
$$

Pick an $\epsilon>0$ in such a way that $\inf _{(x, u) \in T_{k}^{-1}(F)^{-}} \hat{I}_{k}(x, u)<c-2 \epsilon$. Then there exists $\left(x^{*}, u^{*}\right) \in$ $T_{k}^{-1}(F)^{-}$such that $\hat{I}_{k}\left(x^{*}, u^{*}\right)<c-2 \epsilon$. Let $\bar{I}_{k}\left(x_{1}, \ldots, x_{k}, u_{1}, \ldots, u_{k}\right) \triangleq \sum_{i=1}^{k} x_{i}^{\alpha}$. Since $\bar{I}_{k}$ is 
continuous, one can find $\left(x^{\prime}, u^{\prime}\right)=\left(x_{1}^{\prime}, \ldots, x_{k}^{\prime}, u_{1}^{\prime}, \ldots, u_{k}^{\prime}\right) \in T_{k}^{-1}(F)$ sufficiently close to $\left(x^{*}, u^{*}\right)$ so that $\bar{I}_{k}\left(x^{\prime}, u^{\prime}\right)<c-\epsilon$. Note that for any permutation $p:\{1, \ldots, k\} \rightarrow\{1, \ldots, k\},\left(x^{\prime \prime}, u^{\prime \prime}\right) \triangleq$ $\left(x_{p(1)}^{\prime}, \ldots, x_{p(k)}^{\prime}, u_{p(1)}^{\prime}, \ldots, u_{p(k)}^{\prime}\right)$ also belongs to $T_{k}^{-1}(F)$ and $\bar{I}_{k}\left(x^{\prime \prime}, u^{\prime \prime}\right)=\bar{I}_{k}\left(x^{\prime}, u^{\prime}\right)$ due to the symmetric structure of $T_{k}$ and $\bar{I}_{k}$. If we pick $p$ so that $x_{p(1)}^{\prime} \geq \cdots \geq x_{p(k)}^{\prime}$, then $\hat{I}_{k}\left(x^{\prime \prime}, u^{\prime \prime}\right)=$ $\bar{I}_{k}\left(x^{\prime}, u^{\prime}\right)<c-\epsilon \leq \inf _{(x, u) \in T_{k}^{-1}(F)} \hat{I}_{k}(x, u)$, which contradicts to $\left(x^{\prime \prime}, u^{\prime \prime}\right) \in T_{k}^{-1}(F)$. Therefore, (5.20) cannot be the case, which proves the upper bound.

Turning to the lower bound, consider an open set $G \subseteq \mathbb{D}_{\leqslant k}$.

$$
\begin{aligned}
& \liminf _{n \rightarrow \infty} \frac{1}{L(n) n^{\alpha}} \log \mathbf{P}\left(\hat{J}_{n}^{\leqslant k} \in G\right) \\
& =\liminf _{n \rightarrow \infty} \frac{1}{L(n) n^{\alpha}} \log \mathbf{P}\left(\left(Q_{n}^{\leftarrow}\left(\Gamma_{1}\right), \ldots, Q_{n}^{\leftarrow}\left(\Gamma_{k}\right), U_{1}, \ldots, U_{k}\right) \in T_{k}^{-1}(G)\right) \\
& \geq \liminf _{n \rightarrow \infty} \frac{1}{L(n) n^{\alpha}} \log \mathbf{P}\left(\left(Q_{n}^{\leftarrow}\left(\Gamma_{1}\right), \ldots, Q_{n}^{\leftarrow}\left(\Gamma_{k}\right), U_{1}, \ldots, U_{k}\right) \in T_{k}^{-1}(G)^{\circ}\right) \\
& \geq-\inf _{\left(x_{1}, \ldots, x_{k}, u_{1}, \ldots, u_{k}\right) \in T_{k}^{-1}(G)^{\circ}} \hat{I}_{k}\left(x_{1}, \ldots, x_{k}, u_{1}, \ldots, u_{k}\right) .
\end{aligned}
$$

In view of (5.19), we are done if we prove that

$$
\inf _{(x, u) \in T_{k}^{-1}(G)^{\circ}} \hat{I}_{k}(x, u) \leq \inf _{(x, u) \in T_{k}^{-1}(G)} \hat{I}_{k}(x, u) .
$$

Let $(x, u)$ be an arbitrary point in $T_{k}^{-1}(G)$ so that $T_{k}(x, u) \in G$. We will show that there exists $\left(x^{*}, u^{*}\right) \in T_{k}^{-1}(G)^{\circ}$ such that $I_{k}\left(x^{*}, u^{*}\right) \leq I_{k}(x, u)$. Note first that if $u_{i} \in\{0,1\}$ for some $i$, then $x_{i}$ has to be 0 since $G \subseteq \mathbb{D}_{\leqslant k}$. This means that we can replace $u_{i}$ with an arbitrary number in $(0,1)$ without changing the value of $I_{k}$ and $T_{k}$. Therefore, we assume w.l.o.g. that $u_{i}>0$ for each $i=1, \ldots, k$. Now, suppose that $u_{i}=u_{j}$ for some $i \neq j$. Then one can find $\left(x^{\prime}, u^{\prime}\right)$ such that $T_{k}\left(x^{\prime}, u^{\prime}\right)=T_{k}(x, u)$ by setting

$$
\left(x^{\prime}, u^{\prime}\right) \triangleq(x_{1}, \ldots, \underbrace{x_{i}+x_{j}}_{i^{\text {th }}}, \ldots, \underbrace{0}_{j^{\text {th }} \text { coordinate }}, \ldots, x_{k}, u_{1}, \ldots, \underbrace{u_{i}}_{k+i^{\text {th }} \text { coordinate }}, \ldots, \underbrace{u_{j}^{\prime}}_{k+j^{\text {th }} \text { coordinate }}, \ldots, u_{k}),
$$

where $u_{j}^{\prime}$ is an arbitrary number in $(0,1)$; in particular, we can choose $u_{j}^{\prime}$ so that $u_{j}^{\prime} \neq u_{l}$ for $l=$ $1, \ldots, k$. It is easy to see that $\bar{I}_{k}\left(x^{\prime}, u^{\prime}\right) \leq \hat{I}_{k}(x, u)$. Now one can permute the coordinates of $\left(x^{\prime}, u^{\prime}\right)$ as in the upper bound to find $\left(x^{\prime \prime}, u^{\prime \prime}\right)$ such that $T_{k}\left(x^{\prime \prime}, u^{\prime \prime}\right)=T_{k}(x, u)$ and $\hat{I}_{k}\left(x^{\prime \prime}, u^{\prime \prime}\right) \leq \hat{I}_{k}(x, u)$. Iterating this procedure until there is no $i \neq j$ for which $u_{i}=u_{j}$, we can find $\left(x^{*}, u^{*}\right)$ such that $T_{k}\left(x^{*}, u^{*}\right)=T_{k}(x, u), u_{i}^{*}$ 's are all distinct in $(0,1)$, and $I_{k}\left(x^{*}, u^{*}\right) \leq I_{k}(x, u)$. Note that since $T_{k}$ is continuous at $\left(x^{*}, u^{*}\right), T_{k}\left(x^{*}, u^{*}\right) \in G$, and $G$ is open, we conclude that $\left(x^{*}, u^{*}\right) \in T_{k}^{-1}(G)^{\circ}$. Therefore,

$$
\inf _{(x, u) \in T_{k}^{-1}(G)^{\circ}} I_{k}(x, u) \leq I_{k}(x, u) .
$$

Since $(x, u)$ was arbitrarily chosen in $T_{k}^{-1}(G),(5.21)$ is proved. Along with the upper bound, this proves the LDP in $\mathbb{D}_{\leqslant k}$. Finally, since $\mathbb{D}_{\leqslant k}$ is a closed subset of $\mathbb{D}, \mathbf{P}\left(\hat{J}_{n}^{\leqslant k} \notin \mathbb{D}_{\leqslant k}\right)=0$, and $I_{k}=\infty$ on $\mathbb{D} \backslash \mathbb{D}_{\leqslant k}$, Lemma 4.1 .5 of Dembo and Zeitouni (2010) applies, proving the desired LDP in $\mathbb{D}$. 
Now we are ready to prove Lemma 2.1.

Proof of Lemma 2.1. Recall that

$$
\bar{J}_{n}^{k} \stackrel{\mathcal{D}}{=} \underbrace{\frac{1}{n} \sum_{i=1}^{k} Q_{n}^{\leftarrow}\left(\Gamma_{i}\right) \mathbb{1}_{\left[U_{i}, 1\right]}}_{=\hat{J}_{n}^{\leqslant k}}-\underbrace{\frac{1}{n} \mathbb{1}\left\{\tilde{N}_{n}<k\right\} \sum_{i=\tilde{N}_{n}+1}^{k} Q_{n}^{\leftarrow}\left(\Gamma_{i}\right) \mathbb{1}_{\left[U_{i}, 1\right]}}_{=\tilde{J}_{n}^{\leqslant k}} .
$$

Let $F$ be a closed set and note that

$$
\begin{aligned}
\mathbf{P}\left(\bar{J}_{n}^{k} \in F\right) & =\mathbf{P}\left(\hat{J}_{n}^{\leqslant k}-\breve{J}_{n}^{\leqslant k} \in F\right) \leq \mathbf{P}\left(\hat{J}_{n}^{\leqslant k}-\breve{J}_{n}^{\leqslant k} \in F, \mathbb{1}\{N(n)<k\}=0\right)+\mathbf{P}(\mathbb{1}\{N(n)<k\} \neq 0) \\
& \leq \mathbf{P}\left(\hat{J}_{n}^{\leqslant k} \in F\right)+\mathbf{P}(N(n)<k) .
\end{aligned}
$$

From Lemma 5.3,

$$
\begin{aligned}
\limsup _{n \rightarrow \infty} \frac{\log \mathbf{P}\left(\bar{J}_{n}^{k} \in F\right)}{L(n) n^{\alpha}} & \leq \limsup _{n \rightarrow \infty} \frac{\log \mathbf{P}\left(\hat{J}_{n}^{\leqslant k} \in F\right)}{L(n) n^{\alpha}} \vee \limsup _{n \rightarrow \infty} \frac{\log \mathbf{P}(N(n)<k)}{L(n) n^{\alpha}} \\
& \leq-\inf _{\xi \in F} I_{k}(\xi),
\end{aligned}
$$

since $\lim \sup _{n \rightarrow \infty} \frac{1}{L(n) n^{\alpha}} \log \mathbf{P}(N(n)<k)=-\infty$.

Turning to the lower bound, let $G$ be an open set. Since the lower bound is trivial in case $\inf _{x \in G} I_{k}(x)=\infty$, we focus on the case $\inf _{x \in G} I_{k}(x)<\infty$. In this case,

$$
\begin{aligned}
\liminf _{n \rightarrow \infty} \frac{\log \mathbf{P}\left(\bar{J}_{n}^{k} \in G\right)}{L(n) n^{\alpha}} & \geq \liminf _{n \rightarrow \infty} \frac{\log \mathbf{P}\left(\bar{J}_{n}^{k} \in G, N(n) \geq k\right)}{L(n) n^{\alpha}}=\liminf _{n \rightarrow \infty} \frac{\log \mathbf{P}\left(\hat{J}_{n}^{\leqslant k} \in G, N(n) \geq k\right)}{L(n) n^{\alpha}} \\
& \geq \liminf _{n \rightarrow \infty} \frac{1}{L(n) n^{\alpha}} \log \left(\mathbf{P}\left(\hat{J}_{n}^{\leqslant k} \in G\right)-\mathbf{P}(N(n)<k)\right) \\
& =\liminf _{n \rightarrow \infty} \frac{1}{L(n) n^{\alpha}} \log \left(\mathbf{P}\left(\hat{J}_{n}^{\leqslant k} \in G\right)\left(1-\frac{\mathbf{P}(N(n)<k)}{\mathbf{P}\left(\hat{J}_{n}^{\leqslant k} \in G\right)}\right)\right) \\
& =\liminf _{n \rightarrow \infty} \frac{1}{L(n) n^{\alpha}}\left\{\log \left(\mathbf{P}\left(\hat{J}_{n}^{\leqslant k} \in G\right)\right)+\log \left(1-\frac{\mathbf{P}(N(n)<k)}{\mathbf{P}\left(\hat{J}_{n}^{\leqslant k} \in G\right)}\right)\right\} \\
& =\liminf _{n \rightarrow \infty} \frac{1}{L(n) n^{\alpha}} \log \mathbf{P}\left(\hat{J}_{n}^{\leqslant k} \in G\right) \geq-\inf _{\xi \in G} I_{k}(\xi) .
\end{aligned}
$$

The last equality holds since

$$
\lim _{n \rightarrow \infty} \frac{\mathbf{P}(N(n)<k)}{\mathbf{P}\left(\hat{J}_{n}^{\leqslant k} \in G\right)}=\lim _{n \rightarrow \infty}\left\{\exp \left(\frac{\log \mathbf{P}(N(n)<k)}{L(n) n^{\alpha}}-\frac{\log \mathbf{P}\left(\hat{J}_{n}^{\leqslant k} \in G\right)}{L(n) n^{\alpha}}\right)\right\}^{L(n) n^{\alpha}}=0
$$

which in turn follows from

$$
\limsup _{n \rightarrow \infty} \frac{1}{L(n) n^{\alpha}} \log \mathbf{P}(N(n)<k)=-\infty
$$


and

$$
\limsup _{n \rightarrow \infty} \frac{-1}{L(n) n^{\alpha}} \log \mathbf{P}\left(\hat{J}_{n}^{\leqslant k} \in G\right) \leq \inf _{x \in G} I_{k}(x)<\infty
$$

\subsection{Proof of Lemma 2.2.}

Proof of Lemma 2.2. Since the inequality is obvious if $\inf _{\xi \in G} I(\xi)=\infty$, we assume that $\inf _{\xi \in G} I(\xi)<\infty$. Then, there exists a $\xi_{0} \in G$ such that $I\left(\xi_{0}\right) \leq \inf _{\xi \in G} I(\xi)+\delta$. Since $G$ is open, we can pick $\epsilon>0$ such that $B_{J_{1}}\left(\xi_{0} ; 2 \epsilon\right) \subseteq G$ so that $B_{J_{1}}\left(\xi_{0} ; \epsilon\right) \subseteq G^{-\epsilon}$. Note that since $I\left(\xi_{0}\right)<\infty, \xi_{0}$ has the representation $\xi_{0}=\sum_{i=1}^{\infty} x_{i} \mathbb{1}_{\left[u_{i}, 1\right]}$ where $x_{i} \geq 0$ for all $i=1,2, \ldots$, and $u_{i}$ 's all distinct in $(0,1)$. Note also that since $I\left(\xi_{0}\right)=\sum_{i=1}^{\infty} x_{i}^{\alpha}<\infty$ with $\alpha<1, \sum_{i=1}^{\infty} x_{i}$ has to be finite as well. There exists $K$ such that $k \geq K$ implies $\sum_{i=k+1}^{\infty} x_{i}<\epsilon$. For these $\epsilon$ and $K$, we claim that $(2.7)$ holds. For any given $k \geq K$, let $\xi_{1} \triangleq \sum_{i=1}^{k} x_{i} \mathbb{1}_{\left[u_{i}, 1\right]}$, then $I_{k}\left(\xi_{1}\right) \leq I\left(\xi_{0}\right)$ while $d_{J_{1}}\left(\xi_{0}, \xi_{1}\right) \leq\left\|\xi_{0}-\xi_{1}\right\|_{\infty} \leq \sum_{i=k+1}^{\infty} x_{i}<\epsilon$. That is, $\xi_{1} \in B_{J_{1}}\left(\xi_{0} ; \epsilon\right) \subseteq G^{-\epsilon}$. Therefore, $\inf _{\xi \in G^{-\epsilon}} I_{k}(\xi) \leq I\left(\xi_{1}\right) \leq I\left(\xi_{0}\right) \leq \inf _{\xi \in G} I(\xi)+\delta$.

5.5. Proof of Lemma 2.3. In our proof of Lemma 2.3, the following lemmas (Lemma 5.4 and Lemma 5.5) play key roles.

LEMMA 5.4. For each $\epsilon>\delta>0$,

$$
\limsup _{n \rightarrow \infty} \frac{1}{L(n) n^{\alpha}} \log \mathbf{P}\left(\max _{1 \leq j \leq 2 n} \sum_{i=1}^{j}\left(Z_{i} \mathbb{1}_{\left\{Z_{i} \leq n \delta\right\}}-\mathbf{E} Z\right)>n \epsilon\right) \leq-(\epsilon / 3)^{\alpha}(\epsilon / \delta)^{1-\alpha} .
$$

Proof. We refine an argument developed in Jelenković and Momčilović (2003). Note that for any $s>0$ such that $1 / s \leq n \delta$,

$$
\mathbf{E} e^{s Z \mathbb{1}_{\{Z \leq n \delta\}}}=\mathbf{E} e^{s Z \mathbb{1}_{\{Z \leq n \delta\}}} \mathbb{1}_{\left\{Z \geq \frac{1}{s}\right\}}+\mathbf{E} e^{s Z \mathbb{1}_{\{Z \leq n \delta\}}} \mathbb{1}_{\left\{Z<\frac{1}{s}\right\}}=(I)+(I I),
$$


and

$$
\begin{aligned}
(I) & =\int_{[1 / s, n \delta]} e^{s y} \mathrm{~d} \mathbf{P}(Z \leq y)+\int_{(n \delta, \infty)} \mathrm{d} \mathbf{P}(Z \leq y) \\
& =\left[e^{s y} \mathbf{P}(Z \leq y)\right]_{(1 / s)-}^{(n \delta)+}-s \int_{[1 / s, n \delta]} e^{s y} \mathbf{P}(Z \leq y) \mathrm{d} y+\mathbf{P}(Z>n \delta) \\
& =e^{s n \delta} \mathbf{P}(Z \leq n \delta)-e \mathbf{P}(Z<1 / s)-s \int_{[1 / s, n \delta]} e^{s y} \mathrm{~d} y+s \int_{[1 / s, n \delta]} e^{s y} \mathbf{P}(Z>y) \mathrm{d} y+\mathbf{P}(Z>n \delta) \\
& =e^{s n \delta} \mathbf{P}(Z \leq n \delta)-e \mathbf{P}(Z<1 / s)-e^{s n \delta}+e+s \int_{[1 / s, n \delta]} e^{s y} \mathbf{P}(Z>y) \mathrm{d} y+\mathbf{P}(Z>n \delta) \\
& =-e^{s n \delta} \mathbf{P}(Z>n \delta)+e \mathbf{P}(Z \geq 1 / s)+s \int_{[1 / s, n \delta]} e^{s y} \mathbf{P}(Z>y) \mathrm{d} y+\mathbf{P}(Z>n \delta) \\
& \leq s \int_{[1 / s, n \delta]} e^{s y} \mathbf{P}(Z>y) \mathrm{d} y+e \mathbf{P}(Z \geq 1 / s)+\mathbf{P}(Z>n \delta) \\
(5.25) & \leq s \int_{[1 / s, n \delta]} e^{s y} \mathbf{P}(Z>y) \mathrm{d} y+s^{2}(e+1) \mathbf{E} Z^{2},
\end{aligned}
$$

where the last inequality is from $\mathbf{P}(Z \geq n \delta) \leq \mathbf{P}(Z \geq 1 / s) \leq s^{2} \mathbf{E} Z^{2}$; while

$$
(I I) \leq \int_{0}^{1 / s} e^{s y} \mathrm{~d} \mathbf{P}(Z \leq y) \leq \int_{0}^{1 / s}\left(1+s y+(s y)^{2}\right) \mathrm{d} \mathbf{P}(Z \leq y) \leq 1+s \mathbf{E} Z+s^{2} \mathbf{E} Z^{2} .
$$

Therefore, from (5.24), (5.25) and (5.26), if $1 / s \leq n \delta$ and $s$ is sufficiently small,

$$
\begin{aligned}
\mathbf{E} e^{s Z \mathbb{1}_{\{Z \leq n \delta\}}} & \leq s \int_{\frac{1}{s}}^{n \delta} e^{s y} \mathbf{P}(Z>y) d y+1+s \mathbf{E} Z+s^{2}(e+2) \mathbf{E} Z^{2} \\
& =s \int_{\frac{1}{s}}^{n \delta} e^{s y-q(y)} d y+1+s \mathbf{E} Z+s^{2}(e+2) \mathbf{E} Z^{2} \\
& \leq s n \delta e^{s n \delta-q(n \delta)}+1+s \mathbf{E} Z+s^{2}(e+2) \mathbf{E} Z^{2},
\end{aligned}
$$

where $q(x) \triangleq-\log \mathbf{P}(X>x)=L(x) x^{\alpha}$, and the last inequality is from the fact that $e^{s y-q(y)}$ is increasing over $[1 / s, n \delta]$ due to the assumption that $L(y) y^{\alpha-1}$ is non-increasing for sufficiently 
large $y$ 's. Now, from the Markov inequality,

$$
\begin{aligned}
& \mathbf{P}\left(\sum_{i=1}^{j}\left(Z_{i} \mathbb{1}_{\left\{Z_{i} \leq n \delta\right\}}-\mathbf{E} Z\right)>n \epsilon\right) \\
& \leq \mathbf{P}\left(\exp \left(s \sum_{i=1}^{j} Z_{i} \mathbb{1}_{\left\{Z_{i} \leq n \delta\right\}}\right)>\exp (s(n \epsilon+j \mathbf{E} Z))\right) \\
& \leq \exp \left\{-s(n \epsilon+j \mathbf{E} Z)+j \log \left(\mathbf{E} e^{\left.\left.s Z \mathbb{1}_{\{Z \leq n \delta\}}\right)\right\}}\right.\right. \\
& \leq \exp \left\{-s(n \epsilon+j \mathbf{E} Z)+j\left(s n \delta e^{s n \delta-q(n \delta)}+s \mathbf{E} Z+s^{2}(e+2) \mathbf{E} Z^{2}\right)\right\} \\
& =\exp \left\{-s n \epsilon+j s n \delta e^{s n \delta-q(n \delta)}+j s^{2}(e+2) \mathbf{E} Z^{2}\right\} \\
& \leq \exp \left\{-s n \epsilon+2 n^{2} s \delta e^{s n \delta-q(n \delta)}+2 n s^{2}(e+2) \mathbf{E} Z^{2}\right\}
\end{aligned}
$$

for $j \leq 2 n$, where the third inequality is from (5.27) and the generic inequality $\log (x+1) \leq x$. Fix $\gamma \in\left(0,(\epsilon / \delta)^{1-\alpha}\right)$ and and set $s=\frac{\gamma q(n \epsilon)}{n \epsilon}$. Note that $1 / s \rightarrow \infty$ as $n \rightarrow \infty$, while $1 / s \leq n \delta$ for sufficiently large $n$. From now on, we only consider sufficiently large $n$ 's such that $1 / s<n \delta$ and $s$ is sufficiently small so that (5.27) and (5.28) are valid. To establish an upper bound for (5.28), we next examine $e^{s n \delta-q(n \delta)}$. Note that $q(n \epsilon)=q(n \delta) \frac{L(n \epsilon)}{L(n \delta)}(\delta / \epsilon)^{-\alpha}$, and hence,

$$
s n \delta-q(n \delta)=\frac{\gamma q(n \epsilon)}{n \epsilon} n \delta-q(n \delta)=-q(n \delta)\left(1-\gamma \frac{L(n \epsilon)}{L(n \delta)}(\delta / \epsilon)^{1-\alpha}\right),
$$

and

$$
e^{s n \delta-q(n \delta)} \leq e^{-q(n \delta)\left(1-\gamma \frac{L(n \epsilon)}{L(n \delta)}(\delta / \epsilon)^{1-\alpha}\right)}
$$

Plugging this $s\left(=\frac{\gamma q(n \epsilon)}{n \epsilon}\right)$ into (5.28) along with (5.29),

$$
\begin{aligned}
& \max _{0 \leq j \leq 2 n} \mathbf{P}\left(\sum_{i=1}^{j}\left(Z_{i} \mathbb{1}_{\left\{Z_{i} \leq n \delta\right\}}-\mathbf{E} Z\right)>n \epsilon\right) \\
& \leq \exp \left\{-\gamma q(n \epsilon)+\frac{2 \gamma \delta n q(n \epsilon)}{\epsilon} e^{-q(n \delta)\left(1-\gamma \frac{L(n \epsilon)}{L(n \delta)}(\delta / \epsilon)^{1-\alpha}\right)}+\frac{2 \gamma^{2}(e+2) \mathbf{E} Z^{2}}{\epsilon^{2}} \frac{q(n \epsilon)^{2}}{n}\right\} .
\end{aligned}
$$

Since

$$
\limsup _{n \rightarrow \infty} \frac{1}{L(n) n^{\alpha}} \frac{2 \gamma \delta n q(n \epsilon)}{\epsilon} e^{-q(n \delta)\left(1-\gamma \frac{L(n \epsilon)}{L(n \delta)}(\delta / \epsilon)^{1-\alpha}\right)}=0
$$

and

$$
\limsup _{n \rightarrow \infty} \frac{1}{L(n) n^{\alpha}} \frac{2 \gamma^{2}(e+2) \mathbf{E} Z^{2}}{\epsilon^{2}} \frac{q(n \epsilon)^{2}}{n}=0
$$

we conclude that

$$
\limsup _{n \rightarrow \infty} \frac{1}{L(n) n^{\alpha}} \log \max _{0 \leq j \leq 2 n} \mathbf{P}\left(\sum_{i=1}^{j}\left(Z_{i} \mathbb{1}_{\left\{Z_{i} \leq n \delta\right\}}-\mathbf{E} Z\right)>n \epsilon\right)=\limsup _{n \rightarrow \infty} \frac{-\gamma q(n \epsilon)}{L(n) n^{\alpha}}=-\epsilon^{\alpha} \gamma .
$$


From Etemadi's inequality,

$$
\begin{aligned}
& \limsup _{n \rightarrow \infty} \frac{1}{L(n) n^{\alpha}} \log \mathbf{P}\left(\max _{0 \leq j \leq 2 n} \sum_{i=1}^{j}\left(Z_{i} \mathbb{1}_{\left\{Z_{i} \leq n \delta\right\}}-\mathbf{E} Z\right)>3 n \epsilon\right) \\
& \leq \limsup _{n \rightarrow \infty} \frac{1}{L(n) n^{\alpha}} \log \left\{3 \max _{0 \leq j \leq 2 n} \mathbf{P}\left(\sum_{i=1}^{j}\left(Z_{i} \mathbb{1}_{\left\{Z_{i} \leq n \delta\right\}}-\mathbf{E} Z\right)>n \epsilon\right)\right\}=-\epsilon^{\alpha} \gamma .
\end{aligned}
$$

Since this is true for arbitrary $\gamma^{\prime}$ 's such that $\gamma \in\left(0,(\epsilon / \delta)^{1-\alpha}\right)$, we arrive at the conclusion of the lemma.

Lemma 5.5. For every $\epsilon, \delta>0$,

$$
\limsup _{n \rightarrow \infty} \frac{1}{L(n) n^{\alpha}} \log \mathbf{P}\left(\sup _{1 \leq j \leq 2 n} \sum_{i=1}^{j}\left(\mathbf{E} Z-Z_{i} \mathbb{1}_{\left\{Z_{i} \leq n \delta\right\}}\right)>n \epsilon\right)=-\infty .
$$

Proof. Note first that there is $n_{0}$ such that $\mathbf{E}\left(Z_{i} \mathbb{1}_{\left\{Z_{i}>n \delta\right\}}\right) \leq \frac{\epsilon}{3}$ for $n \geq n_{0}$. For $n \geq n_{0}$ and $j \leq 2 n$,

$$
\begin{aligned}
\mathbf{P}\left(\sum_{i=1}^{j}\left(\mathbf{E} Z-Z_{i} \mathbb{1}_{\left\{Z_{i} \leq n \delta\right\}}\right)>n \epsilon\right) & =\mathbf{P}\left(\sum_{i=1}^{j}\left(\mathbf{E} Z \mathbb{1}_{\{Z \leq n \delta\}}-Z_{i} \mathbb{1}_{\left\{Z_{i} \leq n \delta\right\}}\right)>n \epsilon-j \mathbf{E} Z \mathbb{1}_{\{Z>n \delta\}}\right) \\
& \leq \mathbf{P}\left(\sum_{i=1}^{j}\left(\mathbf{E} Z \mathbb{1}_{\{Z \leq n \delta\}}-Z_{i} \mathbb{1}_{\left\{Z_{i} \leq n \delta\right\}}\right)>n \epsilon-j \epsilon / 3\right) \\
& \leq \mathbf{P}\left(\sum_{i=1}^{j}\left(\mathbf{E} Z \mathbb{1}_{\{Z \leq n \delta\}}-Z_{i} \mathbb{1}_{\left\{Z_{i} \leq n \delta\right\}}\right)>\frac{n \epsilon}{3}\right) .
\end{aligned}
$$

Let $Y_{i}^{(n)} \triangleq \mathbf{E}\left(Z_{i} \mathbb{1}_{\left\{Z_{i} \leq n \delta\right\}}\right)-Z_{i} \mathbb{1}_{\left\{Z_{i} \leq n \delta\right\}}$. Recall the definition of $Z$ in Section 2.1 and note that it is bounded from below. Furthermore, $\mathbf{E} Y_{i}^{(n)}=0$, $\operatorname{var} Y_{i}^{(n)} \leq \mathbf{E} Z^{2}$, and $Y_{i}^{(n)} \leq \mathbf{E} Z$ almost surely. From Bennet's inequality,

$$
\begin{aligned}
& \mathbf{P}\left(\sum_{i=1}^{j}\left(\mathbf{E} Z \mathbb{1}_{\left\{Z_{i} \leq n \delta\right\}}-Z_{i} \mathbb{1}_{\left\{Z_{i} \leq n \delta\right\}}\right)>\frac{n \epsilon}{3}\right) \\
& \leq \exp \left[-\frac{j \operatorname{var} Y^{(n)}}{(\mathbf{E} Z)^{2}}\left\{\left(1+\frac{n \epsilon \mathbf{E} Z}{3 j \operatorname{var} Y^{(n)}}\right) \log \left(1+\frac{n \epsilon \mathbf{E} Z}{3 j \operatorname{var} Y^{(n)}}\right)-\left(\frac{n \epsilon \mathbf{E} Z}{3 j \operatorname{var} Y^{(n)}}\right)\right\}\right] \\
& \leq \exp \left[-\frac{j \operatorname{var} Y^{(n)}}{(\mathbf{E} Z)^{2}}\left\{\left(\frac{n \epsilon \mathbf{E} Z}{3 j \operatorname{var} Y^{(n)}}\right) \log \left(1+\frac{n \epsilon \mathbf{E} Z}{3 j \operatorname{var} Y^{(n)}}\right)-\left(\frac{n \epsilon \mathbf{E} Z}{3 j \operatorname{var} Y^{(n)}}\right)\right\}\right] \\
& \leq \exp \left[-\left\{\left(\frac{n \epsilon}{3 \mathbf{E} Z}\right) \log \left(1+\frac{n \epsilon \mathbf{E} Z}{3 j \operatorname{var} Y^{(n)}}\right)-\left(\frac{n \epsilon}{3 \mathbf{E} Z}\right)\right\}\right] \\
& \leq \exp \left[-n\left\{\left(\frac{\epsilon}{3 \mathbf{E} Z}\right) \log \left(1+\frac{\epsilon \mathbf{E} Z}{6 \mathbf{E} Z^{2}}\right)-\left(\frac{\epsilon}{3 \mathbf{E} Z}\right)\right\}\right]
\end{aligned}
$$


for $j \leq 2 n$. Therefore, for $n \geq n_{0}$ and $j \leq 2 n$,

$$
\mathbf{P}\left(\sum_{i=1}^{j}\left(\mathbf{E} Z-Z_{i} \mathbb{1}_{\left\{Z_{i} \leq n \delta\right\}}\right)>n \epsilon\right) \leq \exp \left[-n\left\{\left(\frac{\epsilon}{3 \mathbf{E} Z}\right) \log \left(1+\frac{\epsilon \mathbf{E} Z}{6 \mathbf{E} Z^{2}}\right)-\left(\frac{\epsilon}{3 \mathbf{E} Z}\right)\right\}\right] .
$$

Now, from Etemadi's inequality,

$$
\begin{aligned}
\limsup _{n \rightarrow \infty} & \frac{1}{L(n) n^{\alpha}} \log \mathbf{P}\left(\sup _{1 \leq j \leq 2 n} \sum_{i=1}^{j}\left(\mathbf{E} Z-Z_{i} \mathbb{1}_{\left\{Z_{i} \leq n \delta\right\}}\right)>3 n \epsilon\right) \\
& \leq \limsup _{n \rightarrow \infty} \frac{1}{L(n) n^{\alpha}} \log \left\{3 \max _{1 \leq j \leq 2 n} \mathbf{P}\left(\sum_{i=1}^{j}\left(\mathbf{E} Z-Z_{i} \mathbb{1}_{\left\{Z_{i} \leq n \delta\right\}}\right)>n \epsilon\right)\right\} \\
& \leq \limsup _{n \rightarrow \infty} \frac{1}{L(n) n^{\alpha}} \log \left\{3 \exp \left[-n\left\{\left(\frac{\epsilon}{3 \mathbf{E} Z}\right) \log \left(1+\frac{\epsilon \mathbf{E} Z}{6 \mathbf{E} Z^{2}}\right)-\left(\frac{\epsilon}{3 \mathbf{E} Z}\right)\right\}\right]\right\}=-\infty .
\end{aligned}
$$

Replacing $\epsilon$ with $\epsilon / 3$, we arrive at the conclusion of the lemma.

Now we are ready to prove Lemma 2.3.

Proof of Lemma 2.3.

$$
\begin{aligned}
\mathbf{P}\left(\left\|\bar{H}_{n}^{k}\right\|_{\infty}>\epsilon\right) & \\
\leq \mathbf{P}\left(\left\|\bar{H}_{n}^{k}\right\|_{\infty}>\epsilon,\right. & N(n t) \geq k)+\mathbf{P}\left(\left\|\bar{H}_{n}^{k}\right\|_{\infty}>\epsilon, N(n t)<k\right) \\
\leq \mathbf{P}\left(\left\|\bar{H}_{n}^{k}\right\|_{\infty}>\epsilon,\right. & \left.N(n t) \geq k, Z_{R_{n}^{-1}(k)} \leq n \delta\right) \\
& +\mathbf{P}\left(\left\|\bar{H}_{n}^{k}\right\|_{\infty}>\epsilon, N(n t) \geq k, Z_{R_{n}^{-1}(k)}>n \delta\right)+\mathbf{P}(N(n t)<k) \\
\leq \mathbf{P}\left(\left\|\bar{H}_{n}^{k}\right\|_{\infty}>\epsilon,\right. & \left.N(n t) \geq k, Z_{R_{n}^{-1}(k)} \leq n \delta\right) \\
& +\mathbf{P}\left(N(n t) \geq k, Z_{R_{n}^{-1}(k)}>n \delta\right)+\mathbf{P}(N(n t)<k) .
\end{aligned}
$$

An explicit upper bound for the second term can be obtained:

$$
\begin{aligned}
\mathbf{P}\left(N(n t) \geq k, Z_{R_{n}^{-1}(k)}>n \delta\right) & \leq \mathbf{P}\left(Q_{n}^{\leftarrow}\left(\Gamma_{k}\right)>n \delta\right) \leq \mathbf{P}\left(Q_{n}^{\leftarrow}\left(\Gamma_{k}\right) \geq n \delta\right)=\mathbf{P}\left(\Gamma_{k} \leq Q_{n}(n \delta)\right) \\
& =\int_{0}^{Q_{n}(n \delta)} \frac{1}{k !} t^{k-1} e^{-t} d t=\int_{0}^{n v[n \delta, \infty)} \frac{1}{k !} t^{k-1} e^{-t} d t \leq \int_{0}^{n v(n \delta, \infty)} t^{k-1} d t \\
& =\frac{1}{k} n^{k} e^{-k L(n \delta) n^{\alpha} \delta^{\alpha}} .
\end{aligned}
$$

Therefore,

$$
\limsup _{n \rightarrow \infty} \frac{1}{L(n) n^{\alpha}} \log \mathbf{P}\left(Q_{n}^{\leftarrow}\left(\Gamma_{k}\right) \geq n \delta\right) \leq-k \delta^{\alpha}
$$


Turning to the first term of (5.30), we consider the following decomposition:

$$
\begin{aligned}
& \mathbf{P}\left(\left\|\bar{H}_{n}^{k}\right\|_{\infty}>\epsilon, N(n t) \geq k, Z_{R_{n}^{-1}(k)} \leq n \delta\right) \\
& =\underbrace{\mathbf{P}\left(N(n t) \geq k, Z_{R_{n}^{-1}(k)} \leq n \delta, \sup _{t \in[0,1]} \bar{H}_{n}^{k}(t)>\epsilon\right)}_{\triangleq(\mathrm{i})}+\underbrace{\mathbf{P}\left(N(n t) \geq k, Z_{R_{n}^{-1}(k)} \leq n \delta, \sup _{t \in[0,1]}-\bar{H}_{n}^{k}(t)>\epsilon\right)}_{\triangleq(\mathrm{ii})} .
\end{aligned}
$$

Since $Z_{R_{n}^{-1}(k)} \leq n \delta$ implies $\mathbb{1}_{\left\{R_{n}(i)>k\right\}} \leq \mathbb{1}_{\left\{Z_{i} \leq n \delta\right\}}$

$$
\begin{aligned}
(\mathrm{i}) & \leq \mathbf{P}\left(\sup _{t \in[0,1]} \sum_{i=1}^{N(n t)}\left(Z_{i} \mathbb{1}_{\left\{R_{n}(i)>k\right\}}-\mathbf{E} Z\right)>n \epsilon, N(n t) \geq k, Z_{R_{n}^{-1}(k)} \leq n \delta\right) \\
& \leq \mathbf{P}\left(\sup _{t \in[0,1]} \sum_{i=1}^{N(n t)}\left(Z_{i} \mathbb{1}_{\left\{Z_{i} \leq n \delta\right\}}-\mathbf{E} Z\right)>n \epsilon\right)=\mathbf{P}\left(\sup _{0 \leq j \leq N(n)} \sum_{i=1}^{j}\left(Z_{i} \mathbb{1}_{\left\{Z_{i} \leq n \delta\right\}}-\mathbf{E} Z\right)>n \epsilon\right) \\
& \leq \mathbf{P}\left(\sup _{0 \leq j \leq 2 n} \sum_{i=1}^{j}\left(Z_{i} \mathbb{1}_{\left\{Z_{i} \leq n \delta\right\}}-\mathbf{E} Z\right)>n \epsilon, N(n)<2 n\right)+\mathbf{P}(N(n) \geq 2 n) \\
& \leq \mathbf{P}\left(\sup _{0 \leq j \leq 2 n} \sum_{i=1}^{j}\left(Z_{i} \mathbb{1}_{\left\{Z_{i} \leq n \delta\right\}}-\mathbf{E} Z\right)>n \epsilon\right)+\mathbf{P}(N(n) \geq 2 n) .
\end{aligned}
$$

From Lemma 5.4 and the fact that the second term decays at an exponential rate,

$$
\limsup _{n \rightarrow \infty} \frac{1}{L(n) n^{\alpha}} \mathbf{P}\left(Z_{R_{n}^{-1}(k)} \leq n \delta, \sup _{t \in[0,1]} \bar{H}_{n}^{k}(t)>\epsilon\right) \leq-(\epsilon / 3)^{\alpha}(\epsilon / \delta)^{1-\alpha} .
$$


Turning to (ii),

$$
\begin{aligned}
\text { (ii) } & \leq \mathbf{P}\left(\sup _{t \in[0,1]} \sum_{i=1}^{N(n t)}\left(\mathbf{E} Z-Z_{i} \mathbb{1}_{\left\{R_{n}(i)>k\right\}}\right)>n \epsilon\right) \\
& =\mathbf{P}\left(\sup _{t \in[0,1]} \sum_{i=1}^{N(n t)}\left(\mathbf{E} Z-Z_{i} \mathbb{1}_{\left\{Z_{i} \leq n \delta\right\}}+Z_{i}\left(\mathbb{1}_{\left\{Z_{i} \leq n \delta\right\}}-\mathbb{1}_{\left\{R_{n}(i)>k\right\}}\right)\right)>n \epsilon\right) \\
& \leq \mathbf{P}\left(\sup _{t \in[0,1]} \sum_{i=1}^{N(n t)}\left(\mathbf{E} Z-Z_{i} \mathbb{1}_{\left\{Z_{i} \leq n \delta\right\}}+Z_{i} \mathbb{1}_{\left\{Z_{i} \leq n \delta\right\} \cap\left\{R_{n}(i) \leq k\right\}}\right)>n \epsilon\right) \\
& \leq \mathbf{P}\left(\sup _{t \in[0,1]} \sum_{i=1}^{N(n t)}\left(\mathbf{E} Z-Z_{i} \mathbb{1}_{\left\{Z_{i} \leq n \delta\right\}}\right)+k n \delta>n \epsilon\right) \\
& =\mathbf{P}\left(\sup _{t \in[0,1]} \sum_{i=1}^{N(n t)}\left(\mathbf{E} Z-Z_{i} \mathbb{1}_{\left\{Z_{i} \leq n \delta\right\}}\right)>n(\epsilon-k \delta)\right) \\
& \leq \mathbf{P}\left(\sup _{0 \leq j \leq 2 n} \sum_{i=1}^{j}\left(\mathbf{E} Z-Z_{i} \mathbb{1}_{\left\{Z_{i} \leq n \delta\right\}}\right)>n(\epsilon-k \delta), N(n t)<2 n\right)+\mathbf{P}(N(n t) \geq 2 n) \\
& \leq \mathbf{P}\left(\sup _{0 \leq j \leq 2 n} \sum_{i=1}^{j}\left(\mathbf{E} Z-Z_{i} \mathbb{1}_{\left\{Z_{i} \leq n \delta\right\}}\right)>n(\epsilon-k \delta)\right)+\mathbf{P}(N(n t) \geq 2 n) .
\end{aligned}
$$

Applying Lemma 5.5 to the first term and noticing that the second term vanishes at an exponential rate, we conclude that for $\delta$ and $k$ such that $k \delta<\epsilon$

$$
\limsup _{n \rightarrow \infty} \frac{1}{L(n) n^{\alpha}} \log \mathbf{P}\left(Z_{R_{n}^{-1}(k)} \leq n \delta, \sup _{t \in[0,1]}-\bar{H}_{n}^{k}(t)>\epsilon\right)=-\infty .
$$

From (5.33) and (5.34),

$$
\limsup _{n \rightarrow \infty} \frac{1}{L(n) n^{\alpha}} \log \mathbf{P}\left(Z_{R_{n}^{-1}(k)} \leq n \delta,\left\|\bar{H}_{n}^{k}\right\|_{\infty}>\epsilon\right) \leq-(\epsilon / 3)^{\alpha}(\epsilon / \delta)^{1-\alpha} .
$$

This together with (5.30) and (5.32),

$$
\limsup _{n \rightarrow \infty} \frac{1}{L(n) n^{\alpha}} \mathbf{P}\left(\left\|\bar{H}_{n}^{k}\right\|_{\infty}>\epsilon\right) \leq \max \left\{-(\epsilon / 3)^{\alpha}(\epsilon / \delta)^{1-\alpha},-k \delta^{\alpha}\right\}
$$

for any $\delta$ and $k$ such that $k \delta<\epsilon$. Choosing, for example, $\delta=\frac{\epsilon}{2 k}$ and letting $k \rightarrow \infty$, we arrive at the conclusion of the lemma.

5.6. Proof of Lemma 2.4, 2.5, and 2.6. 
Proof OF Lemma 2.4. We follow a similar program as in the proof of Lemma 2.1. Recall that $\tilde{Q}^{\leftarrow}(x)=\inf \{s>0: \mathbf{P}(Z \geq s)<y\}$ and $V_{(1)}, \ldots, V_{(n-1)}$ are the order statistics of $n-1$ i.i.d. Uniform $(0,1)$ random variables $V_{1}, \ldots, V_{n-1}$. We first claim that $\left(\tilde{Q}^{\leftarrow}\left(V_{(1)}\right) / n, \ldots, \tilde{Q}^{\leftarrow}\left(V_{(k)}\right) / n\right)$ satisfies the LDP with speed $L(n) n^{\alpha}$ and the good rate function $\check{I}_{k}$ defined in (5.5). Let $f$ be a bounded continuous function such that $|f(x)|<M, x \in \mathbb{R}_{+}^{k}$ for some $M \in \mathbb{R}$. We want to prove that

$$
\lim _{n \rightarrow \infty} \frac{1}{L(n) n^{\alpha}} \log \mathbf{E} \exp \left\{L(n) n^{\alpha} f\left(\tilde{Q}^{\leftarrow}\left(V_{(1)}\right) / n, \ldots, \tilde{Q}^{\leftarrow}\left(V_{(k)}\right) / n\right)\right\}=\sup _{x} \Lambda_{f}(x),
$$

where $\Lambda_{f}=f-\check{I}_{k}$ to invoke inverse Varadhan lemma and establish the LDP for $\left(\tilde{Q}^{\leftarrow}\left(V_{(1)}\right) / n, \ldots\right.$, $\left.\tilde{Q}^{\leftarrow}\left(V_{(k)}\right) / n\right)$. Recall that in the proof of Lemma 5.2, we have shown that the supremum of $f(x)-$ $\check{I}_{k}(x)$ over $\mathbb{R}_{+}^{k}$ is attained. Let $\hat{x}$ denote one of the optimizers that attain the supremum. Then, due to the form of $\check{I}_{k}$, for any given $\epsilon>0$, we can find $\delta>0$ and $\check{x}=\left(\check{x}_{1}, \ldots, \check{x}_{k}\right)$ such that $\check{x}_{i} \geq \check{x}_{i+1}+\delta$ for $i=1, \ldots, k-1$ and $x \in \prod_{i=1}^{k}\left[\check{x}_{i}, \breve{x}_{i}+\delta\right]$ implies

$$
\check{I}_{k}(x) \geq \check{I}_{k}(\hat{x})-\epsilon \quad \text { and } \quad f(x)-\check{I}_{k}(x) \geq f(\hat{x})-\check{I}_{k}(\hat{x})-\epsilon .
$$

Therefore, if we set $A_{n}(\delta) \triangleq\left\{\left(y_{1}, \ldots, y_{k}\right): \tilde{Q}^{\leftarrow}\left(y_{i}\right) / n \in\left[\check{x}_{i}, \check{x}_{i}+\delta\right], i=1, \ldots, k+1\right\}$, then $y \in A_{n}(\delta)$ implies

$$
\check{I}_{k}\left(\tilde{Q}^{\leftarrow}\left(y_{1}\right) / n, \ldots, \tilde{Q}^{\leftarrow}\left(y_{k}\right) / n\right) \geq \check{I}_{k}(\hat{x})-\epsilon
$$

and

$$
f\left(\tilde{Q}^{\leftarrow}\left(y_{1}\right) / n, \ldots, \tilde{Q}^{\leftarrow}\left(y_{k}\right) / n\right)-\check{I}_{k}\left(\tilde{Q}^{\leftarrow}\left(y_{1}\right) / n, \ldots, \tilde{Q}^{\leftarrow}\left(y_{k}\right) / n\right) \geq f(\hat{x})-\check{I}_{k}(\hat{x})-\epsilon,
$$

and hence,

$$
f\left(\tilde{Q}^{\leftarrow}\left(y_{1}\right) / n, \ldots, \tilde{Q}^{\leftarrow}\left(y_{k}\right) / n\right) \geq f(\hat{x})-2 \epsilon .
$$

Note also that $\tilde{Q}^{\leftarrow}(y)<x$ if and only if $\mathbf{P}(Z \geq x)<y$, and hence, $\mathbf{P}\left(Z \geq n\left(\check{x}_{i}+\delta\right)\right)<y_{i} \leq$ $\mathbf{P}\left(Z \geq n \check{x}_{i}\right)$ implies $\tilde{Q}^{\leftarrow}\left(y_{i}\right) / n \in\left[\check{x}_{i}, \check{x}_{i}+\delta\right]$. We have that

$$
\mathbb{1}_{\left\{\tilde{Q}^{\leftarrow}\left(y_{i}\right) / n \in\left[\check{x}_{i}, \check{x}_{i}+\delta\right], i=1, \ldots, k\right\}} \geq \mathbb{1}_{\left\{\mathbf{P}\left(Z \geq n\left(\check{x}_{i}+\delta\right)\right)<y_{i} \leq \mathbf{P}\left(Z \geq n \check{x}_{i}\right), i=1, \ldots, k\right\}},
$$

and hence, for $y_{k+1} \geq \mathbf{P}\left(Z \geq n \check{x}_{i}\right)$,

$$
\begin{aligned}
& \int_{0}^{y_{k+1}} \cdots \int_{0}^{y_{2}} \mathbb{1}_{\left\{\tilde{Q}^{\leftarrow}\left(y_{i}\right) / n \in\left[\check{x}_{i}, \check{x}_{i}+\delta\right], i=1, \ldots, k\right\}} d y_{1} \cdots d y_{k} \\
& \geq \int_{0}^{y_{k+1}} \cdots \int_{0}^{y_{2}} \mathbb{1}_{\left\{\mathbf{P}\left(Z \geq n\left(\check{x}_{i}+\delta\right)\right)<y_{i} \leq \mathbf{P}\left(Z \geq n \check{x}_{i}\right), i=1, \ldots, k\right\}} d y_{1} \cdots d y_{k} \\
& =\int_{0}^{1} \cdots \int_{0}^{1} \mathbb{1}_{\left\{\mathbf{P}\left(Z \geq n\left(\check{x}_{i}+\delta\right)\right)<y_{i} \leq \mathbf{P}\left(Z \geq n \check{x}_{i}\right), i=1, \ldots, k\right\}} d y_{1} \cdots d y_{k} \\
& =\prod_{i=1}^{k}\left(\mathbf{P}\left(Z \geq n \check{x}_{i}\right)-\mathbf{P}\left(Z \geq n \check{x}_{i}+n \delta\right)\right) .
\end{aligned}
$$


Therefore,

$$
\begin{aligned}
& \mathbf{E} \exp \left\{L(n) n^{\alpha} f\left(\tilde{Q}^{\leftarrow}\left(V_{(1)}\right) / n, \ldots, \tilde{Q}^{\leftarrow}\left(V_{(k)}\right) / n\right)\right\} \\
& \geq \mathbf{E} \exp \left\{L(n) n^{\alpha} f\left(\tilde{Q}^{\leftarrow}\left(V_{(1)}\right) / n, \ldots, \tilde{Q}^{\leftarrow}\left(V_{(k)}\right) / n\right)\right\} \mathbb{1}_{\left\{\left(V_{(1)}, \ldots, V_{(k)}\right) \in A_{n}(\delta)\right\}} \\
& =\int_{0}^{1} \int_{0}^{y_{n-1}} \cdots \int_{0}^{y_{2}} e^{L(n) n^{\alpha} f\left(\tilde{Q}^{\leftarrow}\left(y_{1}\right) / n, \ldots, \tilde{Q}^{\leftarrow}\left(y_{k}\right) / n\right)} n ! \mathbb{1}_{\left\{\left(y_{1}, \ldots, y_{k}\right) \in A_{n}(\delta)\right\}} d y_{1} \cdots d y_{n-2} d y_{n-1} \\
& \geq n ! e^{L(n) n^{\alpha}(f(\hat{x})-2 \epsilon)} \int_{0}^{1} \int_{0}^{y_{n-1}} \cdots \int_{0}^{y_{2}} \mathbb{1}_{\left\{\tilde{Q}^{\leftarrow}\left(y_{i}\right) / n \in\left[\check{x}_{i}, \check{x}_{i}+\delta\right], i=1, \ldots, k\right\}} d y_{1} \cdots d y_{n-2} d y_{n-1} \\
& \geq n ! e^{L(n) n^{\alpha}(f(\hat{x})-2 \epsilon)} \prod_{i=1}^{k}\left(\mathbf{P}\left(Z \geq n \check{x}_{i}\right)-\mathbf{P}\left(Z \geq n \check{x}_{i}+n \delta\right)\right) \int_{0}^{1} \int_{0}^{y_{n-1}} \cdots \int_{\mathbf{P}\left(Z \geq n \check{x}_{i}\right)}^{y_{k+2}} d y_{k+1} \cdots d y_{n-2} d y_{n-1} \\
& =n ! e^{L(n) n^{\alpha}(f(\hat{x})-2 \epsilon)} \prod_{i=1}^{k}\left(\mathbf{P}\left(Z \geq n \check{x}_{i}\right)-\mathbf{P}\left(Z \geq n \check{x}_{i}+n \delta\right)\right) \frac{1}{(n-k-1) !}\left(1-\mathbf{P}\left(Z \geq n \check{x}_{i}\right)\right)^{n-k-1} .
\end{aligned}
$$

Since

$$
\liminf _{n \rightarrow \infty} \frac{1}{L(n) n^{\alpha}} \log \prod_{i=1}^{k}\left(\mathbf{P}\left(Z \geq n \check{x}_{i}\right)-\mathbf{P}\left(Z \geq n \check{x}_{i}+n \delta\right)\right)=-\sum_{i=1}^{k} \hat{x}^{\alpha}=-\check{I}_{k}(\hat{x})
$$

and

$$
\liminf _{n \rightarrow \infty} \frac{1}{L(n) n^{\alpha}} \log \left(1-\mathbf{P}\left(Z \geq n \check{x}_{i}\right)\right)^{n-k-1}=0
$$

we get

$$
\begin{aligned}
\liminf _{n \rightarrow \infty} \frac{1}{L(n) n^{\alpha}} \log \mathbf{E} e^{L(n) n^{\alpha} f\left(\tilde{Q}^{\leftarrow}\left(V_{(1)}\right) / n, \ldots, \tilde{Q}^{\leftarrow}\left(V_{(k)}\right) / n\right)} & \geq f(\hat{x})-2 \epsilon-\check{I}_{k}(\hat{x}) \\
& =\sup _{x \in \mathbb{R}_{+}^{k}}\left\{f(x)-\check{I}_{k}(x)\right\}-2 \epsilon .
\end{aligned}
$$

Letting $\epsilon \rightarrow 0$, we arrive at the lower bound.

Turning to the upper bound,

$$
\begin{aligned}
\mathbf{E} \exp \{ & \left.L(n) n^{\alpha} f\left(\tilde{Q}^{\leftarrow}\left(V_{(1)}\right) / n, \ldots, \tilde{Q}^{\leftarrow}\left(V_{(k)}\right) / n\right)\right\} \\
=\mathbf{E} \exp \left\{L(n) n^{\alpha} f\left(\tilde{Q}^{\leftarrow}\left(V_{(1)}\right) / n, \ldots, \tilde{Q}^{\leftarrow}\left(V_{(k)}\right) / n\right)\right\} \mathbb{1}_{\left\{\tilde{Q}^{\leftarrow}\left(V_{(1)}\right) / n>R\right\}} & \\
& +\mathbf{E} \exp \left\{L(n) n^{\alpha} f\left(\tilde{Q}^{\leftarrow}\left(V_{(1)}\right) / n, \ldots, \tilde{Q}^{\leftarrow}\left(V_{(k)}\right) / n\right)\right\} \mathbb{1}_{\left\{\tilde{Q}^{\leftarrow}\left(V_{(1)}\right) / n \leq R\right\}}
\end{aligned}
$$

For the first term, note that

$$
\begin{aligned}
& \mathbf{E} \exp \left\{L(n) n^{\alpha} f\left(\tilde{Q}^{\leftarrow}\left(V_{(1)}\right) / n, \ldots, \tilde{Q}^{\leftarrow}\left(V_{(k)}\right) / n\right)\right\} \mathbb{1}_{\left\{\tilde{Q}^{\leftarrow}\left(V_{(1)}\right) / n>R\right\}} \\
& \leq \mathbf{E} \exp \left\{L(n) n^{\alpha} M\right\} \mathbb{1}\left\{\tilde{Q}^{\leftarrow}\left(V_{(1)}\right) / n>R\right\} \leq \exp \left\{L(n) n^{\alpha} M\right\} \mathbf{P}\left(V_{(1)} \leq \mathbf{P}(Z \geq n R)\right) \\
& =\exp \left\{L(n) n^{\alpha} M\right\}\left(1-(1-\mathbf{P}(Z \geq n R))^{n-1}\right) \\
& =\exp \left\{L(n) n^{\alpha} M\right\}\left(1-\left(1-\exp \left\{-L(n R)(n R)^{\alpha}\right\}\right)^{n-1}\right) .
\end{aligned}
$$


Also, from the generic inequality $1-\exp (-z) \leq z$,

$$
\begin{aligned}
1-(1-1 / x)^{y} & =1-\left\{(1-1 / x)^{x}\right\}^{y / x}=1-\exp \log \left\{(1-1 / x)^{x}\right\}^{y / x}=1-\exp \left\{(y / x) \log (1-1 / x)^{x}\right\} \\
& \leq(y / x) \log (1-1 / x)^{-x}
\end{aligned}
$$

for any $x, y>0$. Setting $x=\exp \left(L(n R)(n R)^{\alpha}\right)$ and $y=n-1$, we get

$$
\begin{aligned}
& 1-\left(1-\exp \left\{-L(n R)(n R)^{\alpha}\right\}\right)^{n-1} \\
& \leq(n-1) \exp \left\{-L(n R)(n R)^{\alpha}\right\} \log \left(1-1 / \exp \left\{L(n R)(n R)^{\alpha}\right\}\right)^{-\exp \left\{L(n R)(n R)^{\alpha}\right\}} .
\end{aligned}
$$

Substituting this into (5.36), we arrive at the upper bound for the first term:

$$
\limsup _{n \rightarrow \infty} \frac{1}{L(n) n^{\alpha}} \log \mathbf{E} \exp \left\{L(n) n^{\alpha} f\left(\tilde{Q}^{\leftarrow}\left(V_{(1)}\right) / n, \ldots, \tilde{Q}^{\leftarrow}\left(V_{(k)}\right) / n\right)\right\} \mathbb{1}_{\left\{\tilde{Q}^{\leftarrow}\left(V_{(1)}\right) / n>R\right\}} \leq M-R^{\alpha} .
$$

For the second term, fix $\epsilon>0$ and pick $\left\{\check{x}^{(1)}, \ldots, \check{x}^{(m)}\right\} \subset \mathbb{R}_{+}^{k}$ in such a way that

$$
\left\{\prod_{j=1}^{k}\left[\check{x}_{j}^{(l)}-\epsilon, \check{x}_{j}^{(l)}+\epsilon\right]\right\}_{l=1, \ldots, m}
$$

covers $\left\{\left(x_{1}, \ldots, x_{k}\right): R \geq x_{1} \geq x_{2} \geq \cdots \geq x_{k} \geq 0\right\}$ and $\check{x}_{1}^{(l)} \geq \check{x}_{2}^{(l)} \geq \cdots \geq \check{x}_{k}^{(l)} \geq 0$ for $l=1, \ldots, m$. Set

$$
A_{n, l}(R) \triangleq\left\{\left(y_{1}, \ldots, y_{k}\right) \in \mathbb{R}_{+}^{k}: y_{1} \leq \cdots \leq y_{k}, Q^{\leftarrow}\left(y_{j}\right) / n \in\left[\check{x}_{j}^{(l)}-\epsilon, \check{x}_{j}^{(l)}+\epsilon\right], j=1, \ldots, k\right\} .
$$

Note that $y_{1} \leq \cdots \leq y_{k} \& Q^{\leftarrow}\left(y_{1}\right) / n \leq R$ implies $R \geq Q^{\leftarrow}\left(y_{1}\right) / n \geq Q^{\leftarrow}\left(y_{2}\right) / n \geq \cdots \geq Q^{\leftarrow}\left(y_{k}\right) / n$, which, in turn, implies $Q^{\leftarrow}\left(y_{j}\right) / n \in\left[\check{x}_{j}^{(l)}-\epsilon, \check{x}_{j}^{(l)}+\epsilon\right], j=1, \ldots, k$ for some $l \in\{1, \ldots, m\}$. Therefore, $\left\{\left(y_{1}, \ldots, y_{k}\right) \in \mathbb{R}_{+}^{k}: y_{1} \leq \cdots \leq y_{k}, Q^{\leftarrow}\left(y_{1}\right) / n \leq R\right\} \subseteq \bigcup_{l=1}^{m} A_{n, l}(R)$, and hence,

$$
\begin{aligned}
& \mathbf{E} \exp \left\{L(n) n^{\alpha} f\left(\tilde{Q}^{\leftarrow}\left(V_{(1)}\right) / n, \ldots, \tilde{Q}^{\leftarrow}\left(V_{(k)}\right) / n\right)\right\} \mathbb{1}_{\left\{\tilde{Q}^{\leftarrow}\left(V_{(1)}\right) \leq R\right\}} \\
& \leq \sum_{i=1}^{m} \mathbf{E} \exp \left\{L(n) n^{\alpha} f\left(\tilde{Q}^{\leftarrow}\left(V_{(1)}\right) / n, \ldots, \tilde{Q}^{\leftarrow}\left(V_{(k)}\right) / n\right)\right\} \mathbb{1}_{\left\{\left(V_{(1)}, \ldots, V_{(k)}\right) \in A_{n, l}(R)\right\}}
\end{aligned}
$$

Note that

$$
\begin{aligned}
& \mathbf{E} \exp \left\{L(n) n^{\alpha} f\left(\tilde{Q}^{\leftarrow}\left(V_{(1)}\right) / n, \ldots, \tilde{Q}^{\leftarrow}\left(V_{(k)}\right) / n\right)\right\} \mathbb{1}_{\left\{\left(V_{(1)}, \ldots, V_{(k)}\right) \in A_{n, l}(R)\right\}} \\
& =\mathbf{E} e^{L(n) n^{\alpha} \Lambda_{f}\left(\tilde{Q}^{\leftarrow}\left(V_{(1)}\right) / n, \ldots, \tilde{Q}^{\leftarrow}\left(V_{(k)}\right) / n\right)} e^{L(n) n^{\alpha} \check{I}_{k}\left(\tilde{Q}^{\leftarrow}\left(V_{(1)}\right) / n, \ldots, \tilde{Q}^{\leftarrow}\left(V_{(k)}\right) / n\right)} \mathbb{1}_{\left\{\left(V_{(1)}, \ldots, V_{(k)}\right) \in A_{n, l}(R)\right\}} \\
& \leq e^{L(n) n^{\alpha} \Lambda_{f}\left(\hat{x}_{1}, \ldots, \hat{x}_{k}\right)} \mathbf{E} e^{L(n) n^{\alpha} \check{I}_{k}\left(\tilde{Q}^{\leftarrow}\left(V_{(1)}\right) / n, \ldots, \tilde{Q}^{\leftarrow}\left(V_{(k)}\right) / n\right)} \mathbb{1}_{\left\{\left(V_{(1)}, \ldots, V_{(k)}\right) \in A_{n, l}(R)\right\}} \\
& \leq e^{L(n) n^{\alpha} \Lambda_{f}\left(\hat{x}_{1}, \ldots, \hat{x}_{k}\right)} e^{L(n) n^{\alpha} \check{I}_{k}\left(\check{x}_{1}^{(l)}+\epsilon, \ldots, \check{x}_{k}^{(l)}+\epsilon\right)} \mathbf{E} \mathbb{1}_{\left\{\left(V_{(1)}, \ldots, V_{(k)}\right) \in A_{n, l}(R)\right\}}
\end{aligned}
$$


and

$$
\begin{aligned}
& \mathbf{E} \mathbb{1}_{\left\{\left(V_{(1)}, \ldots, V_{(k)}\right) \in A_{n, l}(R)\right\}} \\
& \int_{0}^{1} \int_{0}^{y_{n-1}} \cdots \int_{0}^{y_{2}}(n-1) ! \mathbb{1}_{\left\{\left(y_{1}, \ldots, y_{k}\right) \in A_{n, l}(R)\right\}} d y_{1} \cdots d y_{n-2} d y_{n-1} \\
& \geq(n-1) ! \int_{0}^{1} \int_{0}^{y_{n-1}} \cdots \int_{0}^{y_{2}} \mathbb{1}_{\left\{\tilde{Q}^{\leftarrow}\left(y_{i}\right) / n \in\left[\check{x}_{i}^{(l)}-\epsilon, \check{x}_{i}^{(l)}+\epsilon\right], i=1, \ldots, k\right\}} d y_{1} \cdots d y_{n-2} d y_{n-1} \\
& \leq(n-1) ! \prod_{i=1}^{k}\left(\mathbf{P}\left(Z \geq n \check{x}_{i}^{(l)}-n \epsilon\right)-\mathbf{P}\left(Z \geq n \check{x}_{i}^{(l)}+n \epsilon\right)\right) \int_{0}^{1} \int_{0}^{y_{n-1}} \cdots \int_{0}^{y_{k+2}} d y_{k+1} \cdots d y_{n-2} d y_{n-1} \\
& =(n-1) ! \prod_{i=1}^{k}\left(\mathbf{P}\left(Z \geq n \check{x}_{i}^{(l)}-n \epsilon\right)-\mathbf{P}\left(Z \geq n \check{x}_{i}^{(l)}+n \epsilon\right)\right) \frac{1}{(n-k-1) !} . \\
& \leq n^{k} \prod_{i=1}^{k}\left(\mathbf{P}\left(Z \geq n \check{x}_{i}^{(l)}-n \epsilon\right)-\mathbf{P}\left(Z \geq n \check{x}_{i}^{(l)}+n \epsilon\right)\right) .
\end{aligned}
$$

Therefore,

$$
\begin{aligned}
& \mathbf{E} \exp \left\{L(n) n^{\alpha} f\left(\tilde{Q}^{\leftarrow}\left(V_{(1)}\right) / n, \ldots, \tilde{Q}^{\leftarrow}\left(V_{(k)}\right) / n\right)\right\} \mathbb{1}_{\left\{\tilde{Q}^{\leftarrow}\left(V_{(1)}\right) \leq R\right\}} \\
& \leq n^{k} e^{L(n) n^{\alpha} \Lambda_{f}\left(\hat{x}_{1}, \ldots, \hat{x}_{k}\right)} \sum_{i=1}^{l} e^{L(n) n^{\alpha} \check{I}_{k}\left(\check{x}_{1}^{(l)}+\epsilon, \ldots, \check{x}_{k}^{(l)}+\epsilon\right)} \prod_{i=1}^{k}\left(\mathbf{P}\left(Z \geq n \check{x}_{i}^{(l)}-n \epsilon\right)-\mathbf{P}\left(Z \geq n \check{x}_{i}^{(l)}+n \epsilon\right)\right) .
\end{aligned}
$$

Note that

$$
\begin{aligned}
& \limsup _{n \rightarrow \infty} \frac{1}{L(n) n^{\alpha}} \log \prod_{i=1}^{k}\left(\mathbf{P}\left(Z \geq n \check{x}_{i}^{(l)}-n \epsilon\right)-\mathbf{P}\left(Z \geq n \check{x}_{i}^{(l)}+n \epsilon\right)\right) \\
& =-\sum_{i=1}^{k}\left(\check{x}_{i}^{(l)}-\epsilon\right)_{+}^{\alpha}=-\check{I}_{k}\left(\left(\check{x}_{1}^{(l)}-\epsilon, \ldots, \check{x}_{k}^{(l)}-\epsilon\right)_{+}\right),
\end{aligned}
$$

where $(y)_{+}$denotes $\max \{y, 0\}$ and $\left(y_{1}, \ldots, y_{k}\right)_{+}$denotes $\left(\left(y_{1}\right)_{+}, \ldots,\left(y_{k}\right)_{+}\right)$. This along with the principle of the largest term,

$$
\begin{aligned}
& \limsup _{n \rightarrow \infty} \frac{1}{L(n) n^{\alpha}} \log \mathbf{E} \exp \left\{L(n) n^{\alpha} f\left(\tilde{Q}^{\leftarrow}\left(V_{(1)}\right) / n, \ldots, \tilde{Q}^{\leftarrow}\left(V_{(k)}\right) / n\right)\right\} \mathbb{1}_{\left\{\tilde{Q}^{\leftarrow}\left(V_{(1)}\right) \leq R\right\}} \\
& \leq \max _{l=1, \ldots, m}\left(\Lambda_{f}\left(\hat{x}_{1}, \ldots, \hat{x}_{k}\right)+\check{I}_{k}\left(\check{x}_{1}^{(l)}+\epsilon, \ldots, \check{x}_{k}^{(l)}+\epsilon\right)-\check{I}_{k}\left(\left(\check{x}_{1}^{(l)}-\epsilon, \ldots, \check{x}_{k}^{(l)}-\epsilon\right)_{+}\right)\right) \\
& \leq \max _{l=1, \ldots, m}\left(\Lambda_{f}\left(\hat{x}_{1}, \ldots, \hat{x}_{k}\right)+k \epsilon^{\alpha}\right) .
\end{aligned}
$$

Sending $\epsilon \rightarrow 0$, we get

$$
\begin{aligned}
& \limsup _{n \rightarrow \infty} \frac{1}{L(n) n^{\alpha}} \log \mathbf{E} \exp \left\{L(n) n^{\alpha} f\left(\tilde{Q}^{\leftarrow}\left(V_{(1)}\right) / n, \ldots, \tilde{Q}^{\leftarrow}\left(V_{(k)}\right) / n\right)\right\} \mathbb{1}_{\left\{\tilde{Q}^{\leftarrow}\left(V_{(1)}\right) \leq R\right\}} \\
& \leq \Lambda_{f}\left(\hat{x}_{1}, \ldots, \hat{x}_{k}\right) .
\end{aligned}
$$


Now, combining with the bound for the first term, and sending $R \rightarrow \infty$, we get the upper bound:

$$
\begin{aligned}
& \limsup _{n \rightarrow \infty} \frac{1}{L(n) n^{\alpha}} \log \mathbf{E} \exp \left\{L(n) n^{\alpha} f\left(\tilde{Q}^{\leftarrow}\left(V_{(1)}\right) / n, \ldots, \tilde{Q}^{\leftarrow}\left(V_{(k)}\right) / n\right)\right\} \\
& \leq \max \left\{\Lambda_{f}\left(\hat{x}_{1}, \ldots, \hat{x}_{k}\right), M-R^{\alpha}\right\} \rightarrow \Lambda_{f}\left(\hat{x}_{1}, \ldots, \hat{x}_{k}\right) .
\end{aligned}
$$

Together with the lower bound, we get

$$
\lim _{n \rightarrow \infty} \frac{1}{L(n) n^{\alpha}} \log \mathbf{E} \exp \left\{L(n) n^{\alpha} f\left(\tilde{Q}^{\leftarrow}\left(V_{(1)}\right) / n, \ldots, \tilde{Q}^{\leftarrow}\left(V_{(k)}\right) / n\right)\right\}=\Lambda_{f}\left(\hat{x}_{1}, \ldots, \hat{x}_{k}\right),
$$

which in turn allows us to apply Bryc's inverse Varadhan Lemma to prove that $\left(\tilde{Q}^{\leftarrow}\left(V_{(1)}\right) / n, \ldots\right.$, $\left.\tilde{Q}^{\leftarrow}\left(V_{(k)}\right) / n\right)$ satisfies the LDP with the rate function $\check{I}_{k}$. From Theorem 4.14 of Ganesh, O'Connell and Wischik (2004), we see that $\left(\tilde{Q}^{\leftarrow}\left(V_{(1)}\right) / n, \ldots, \tilde{Q}^{\leftarrow}\left(V_{(k)}\right) / n, Z / n\right)$ satisfies the LDP with the rate function $\check{I}_{k}^{\prime}$ given by

$$
\check{I}_{k}^{\prime}\left(x_{1}, \ldots, x_{k+1}\right)=\left\{\begin{array}{ll}
\sum_{i=1}^{k+1} x_{i}^{\alpha} & \text { if } x_{1} \geq x_{2} \geq \cdots \geq x_{k} \geq 0 \text { and } x_{k+1} \geq 0 \\
\infty, & \text { o.w. }
\end{array} .\right.
$$

Proceeding with essentially the same argument as in Corollary 5.1 and Lemma 5.3 - except for considering a mapping $\tilde{T}_{k}:\left(x_{1}, \ldots, x_{k+1}, u_{1}, \ldots, u_{k}\right) \mapsto \sum_{i=1}^{k} x_{i} \mathbb{1}_{\left[u_{i}, 1\right]}+x_{k+1} \mathbb{1}_{\{1\}}$ instead of the mapping $\left.T_{k}:\left(x_{1}, \ldots, x_{k}, u_{1}, \ldots, u_{k}\right) \mapsto \sum_{i=1}^{k} x_{i} \mathbb{1}_{\left[u_{i}, 1\right]}\right)$ and $\tilde{\mathbb{D}}_{\leqslant k}$ instead of $\mathbb{D}_{\leqslant k}$-we conclude that $\tilde{J}_{n}^{k}(t)=\frac{1}{n} \sum_{i=1}^{k} \tilde{Q}^{\leftarrow}\left(V_{(i)}\right) \mathbb{1}_{\left[U_{i}, 1\right]}(t)+\frac{1}{n} Z \mathbb{1}_{\{1\}}(t)$ satisfies the LDP with speed $L(n) n^{\alpha}$ and the rate function $\tilde{I}_{k}$ in (2.10).

Proof of Lemma 2.5. The proof is essentially identical to Lemma 2.2, and hence, omitted.

Proof of Lemma 2.6. Let

$$
\check{H}_{n}^{k}(t) \triangleq \tilde{H}_{n}^{k}(t)+\frac{1}{n} \mathbf{E} Z \mathbb{1}_{\{1\}}(t)=\frac{1}{n} \sum_{i=k+1}^{n-1} \tilde{Q}^{\leftarrow}\left(V_{(i)}\right) \mathbb{1}_{\left[U_{i}, 1\right]}(t)-\frac{1}{n} \sum_{i=1}^{n-1} \mathbf{E} Z \mathbb{1}_{\left[U_{i}, 1\right]}(t) .
$$

Since $\mathbf{P}\left(\left\|\tilde{H}_{n}^{k}\right\|_{\infty} \geq \epsilon\right) \leq \mathbf{P}\left(\left\|\tilde{H}_{n}^{k}\right\|_{\infty} \geq \epsilon / 2\right)+\mathbf{P}\left(\left\|\frac{1}{n} \mathbf{E} Z \sum_{i=1}^{n-1} \mathbb{1}_{\left[U_{i}, 1\right]}+\frac{1}{n} \mathbf{E} Z \mathbb{1}_{\{1\}}\right\|_{\infty} \geq \epsilon / 2\right)$ and $\mathbf{P}\left(\left\|\frac{1}{n} \mathbf{E} Z \sum_{i=1}^{n-1} \mathbb{1}_{\left[U_{i}, 1\right]}(t)+\frac{1}{n} \mathbf{E} Z \mathbb{1}_{\{1\}}\right\|_{\infty}>\epsilon / 2\right)=0$ for large enough $n$, we only need to prove that

$$
\limsup _{k \rightarrow \infty} \limsup _{n \rightarrow \infty} \frac{1}{L(n) n^{\alpha}} \log \mathbf{P}\left(\left\|\check{H}_{n}^{k}\right\|_{\infty}>\epsilon\right)=-\infty
$$

To show this, we fix an arbitrary $\delta \in(0, \epsilon / k)$ and consider the following decomposition:

$$
\mathbf{P}\left(\left\|\check{H}_{n}^{k}\right\|_{\infty}>\epsilon\right) \leq \mathbf{P}\left(\left\|\check{H}_{n}^{k}\right\|_{\infty}>\epsilon, \tilde{Q}^{\leftarrow}\left(V_{(k)}\right)<n \delta\right)+\mathbf{P}\left(\tilde{Q}^{\leftarrow}\left(V_{(k)}\right) \geq n \delta\right)
$$


We first bound the second term. Since the density of the $k^{\text {th }}$ order statistic of the uniform distribution on [0,1] is $n\left(\begin{array}{l}n-1 \\ k-1\end{array}\right) x^{k-1}(1-x)^{n-k}$,

$$
\begin{aligned}
\mathbf{P}\left(\tilde{Q}^{\leftarrow}\left(V_{(k)}\right) \geq n \delta\right) & =\mathbf{P}\left(V_{(k)} \leq \mathbf{P}(Z \geq n \delta)\right) \leq \int_{0}^{\mathbf{P}(Z \geq n \delta)} n\left(\begin{array}{l}
n-1 \\
k-1
\end{array}\right) x^{k-1} d x \\
& =\left(\begin{array}{l}
n \\
k
\end{array}\right)(\mathbf{P}(Z \geq n \delta))^{k}=\left(\begin{array}{l}
n \\
k
\end{array}\right) \exp \left(-k L(n \delta)(n \delta)^{\alpha}\right)
\end{aligned}
$$

and hence, $\lim \sup _{n \rightarrow \infty} \frac{1}{L(n) n^{\alpha}} \log \mathbf{P}\left(\tilde{Q}_{n}^{\leftarrow}\left(V_{(k)}\right)>n \delta\right) \leq-k \delta^{\alpha}$. For the first term

$$
\begin{aligned}
& \mathbf{P}\left(\left\|\check{H}_{n}^{k}\right\|_{\infty}>\epsilon, \tilde{Q}^{\leftarrow}\left(V_{(k)}\right)<n \delta\right) \\
& =\mathbf{P}\left(\sup _{t \in[0,1]} \check{H}_{n}^{k}(t)>\epsilon, \tilde{Q}^{\leftarrow}\left(V_{(k)}\right) \leq n \delta\right)+\mathbf{P}\left(\sup _{t \in[0,1]}-\check{H}_{n}^{k}>\epsilon, \tilde{Q}^{\leftarrow}\left(V_{(k)}\right) \leq n \delta\right) \\
& \leq \mathbf{P}\left(\max _{1 \leq j \leq n-1} \sum_{i=1}^{j}\left(Z_{i} \mathbb{1}_{\left\{Z_{i} \leq n \delta\right\}}-\mathbf{E} Z\right)>n \epsilon\right)+\mathbf{P}\left(\max _{1 \leq j \leq n-1} \sum_{i=1}^{j}\left(\mathbf{E} Z-Z_{i} \mathbb{1}_{\left\{Z_{i} \leq n \delta\right\}}\right)+k n \delta>n \epsilon\right) .
\end{aligned}
$$

Note that from Lemma 5.4,

$$
\limsup _{n \rightarrow \infty} \frac{1}{L(n) n^{\alpha}} \mathbf{P}\left(\max _{1 \leq j \leq n-1} \sum_{i=1}^{j}\left(Z_{i} \mathbb{1}_{\left\{Z_{i} \leq n \delta\right\}}-\mathbf{E} Z\right)>n \epsilon\right) \leq-(\epsilon / 3)^{\alpha}(\epsilon / \delta)^{1-\alpha}
$$

and from 5.5, since $\delta<\epsilon / k$,

$$
\limsup _{n \rightarrow \infty} \frac{1}{L(n) n^{\alpha}} \mathbf{P}\left(\max _{1 \leq j \leq n-1} \sum_{i=1}^{j}\left(\mathbf{E} Z-Z_{i} \mathbb{1}_{\left\{Z_{i} \leq n \delta\right\}}\right)+k n \delta>n \epsilon\right)=-\infty .
$$

Therefore,

$\limsup _{n \rightarrow \infty} \frac{1}{L(n) n^{\alpha}} \mathbf{P}\left(\left\|\tilde{H}_{n}^{k}\right\|_{\infty}>\epsilon, Q_{n}^{\leftarrow}\left(V_{(k)}\right) \leq n \delta\right) \leq \max \left\{-(\epsilon / 3)^{\alpha}(\epsilon / \delta)^{1-\alpha},-\infty\right\}=-(\epsilon / 3)^{\alpha}(\epsilon / \delta)^{1-\alpha}$

Applying the principle of the maximum term once again,

$$
\lim _{k \rightarrow \infty} \limsup _{n \rightarrow \infty} \frac{1}{L(n) n^{\alpha}} \mathbf{P}\left(\left\|\tilde{H}_{n}^{k}\right\|_{\infty}>\epsilon\right) \leq \lim _{k \rightarrow \infty} \max \left\{-(\epsilon / 3)^{\alpha}(\epsilon / \delta)^{1-\alpha},-k \delta^{\alpha}\right\}=-(\epsilon / 3)^{\alpha}(\epsilon / \delta)^{1-\alpha} .
$$

Since $\delta$ can be chosen arbitrarily small,

$$
\lim _{k \rightarrow \infty} \limsup _{n \rightarrow \infty} \frac{1}{L(n) n^{\alpha}} \mathbf{P}\left(\left\|\tilde{H}_{n}^{k}\right\|_{\infty}>\epsilon\right)=-\infty .
$$


5.7. Proof of Theorem 2.3. We follow a similar program as in Section 2.1 and the earlier subsections of this section. Let $\bar{Q}_{n}^{(i)}(j) \triangleq Q_{n}^{\leftarrow}\left(\Gamma_{j}^{(i)}\right) / n$ where $Q_{n}^{\leftarrow}(t)=\inf \{s>0: n \nu[s, \infty)<t\}$ and $\Gamma_{l}^{(i)}=E_{1}^{(i)}+\cdots+E_{l}^{(i)}$ where $E_{j}^{(i)}$ 's are independent standard exponential random variables. Let $U_{j}^{(i)}$ be independent uniform random variables in $[0,1]$ and $Z_{n}^{(i)} \triangleq\left(\bar{Q}_{n}^{(i)}(1), \ldots, \bar{Q}_{n}^{(i)}(k)\right.$, $\left.U_{1}^{(i)}, \ldots, U_{k}^{(i)}\right)$. The following corollary is an immediate consequence of Corollary 5.1 and Theorem 4.14 of Ganesh, O'Connell and Wischik (2004).

Corollary 5.2. $\quad\left(Z_{n}^{(1)}, \ldots, Z_{n}^{(d)}\right)$ satisfies the LDP in $\prod_{i=1}^{d}\left(\mathbb{R}_{+}^{k} \times[0,1]^{k}\right)$ with the rate function $\hat{I}_{k}^{d}\left(z^{(1)}, \ldots, z^{(d)}\right) \triangleq \sum_{j=1}^{d} \hat{I}_{k}\left(z^{(j)}\right)$ where $z^{(j)}=\left(x_{1}^{(j)}, \ldots, x_{k}^{(j)}, u_{1}^{(j)}, \ldots, u_{k}^{(j)}\right)$ for each $j \in\{1, \ldots, d\}$.

Let $\hat{J}_{n}^{\leqslant k(i)} \triangleq \sum_{j=1}^{k} \bar{Q}_{n}^{(i)}(j) \mathbb{1}_{\left[U_{j}^{(i)}, 1\right]}$.

Lemma 5.6. $\quad\left(\hat{J}_{n}^{\leqslant k(1)}, \ldots, \hat{J}_{n}^{\leqslant k(d)}\right)$ satisfies the LDP in $\prod_{i=1}^{d} \mathbb{D}([0,1], \mathbb{R})$ with speed $L(n) n^{\alpha}$ and the rate function

$$
I_{k}^{d}\left(\xi_{1}, \ldots \xi_{d}\right) \triangleq \sum_{i=1}^{d} I_{k}\left(\xi_{i}\right)= \begin{cases}\sum_{i=1}^{d} \sum_{t: \xi_{i}(t) \neq \xi_{i}(t-)}\left(\xi_{i}(t)-\xi_{i}(t-)\right)^{\alpha} & \text { if } \xi_{i} \in \mathbb{D}_{\leqslant k} \text { for } i=1, \ldots, d \\ \infty, & \text { otherwise }\end{cases}
$$

Proof. Since $I_{k_{i}}$ is lower semi-continuous in $\prod_{i=1}^{d} \mathbb{D}([0,1], \mathbb{R})$ for each $i, I_{k_{1}, \ldots, k_{d}}$ is a sum of lower semi-continuous functions, and hence, is lower semi-continuous itself. The rest of the proof for the LDP upper bound and the lower bounds mirrors that of the one dimensional case (Lemma 5.3) closely, and hence, omitted.

Proof of Lemma 2.7. Again, we consider the same distributional relation for each coordinate as in the 1-dimensional case:

$$
\bar{J}_{n}^{k(i)} \stackrel{\mathcal{D}}{=} \underbrace{\frac{1}{n} \sum_{j=1}^{k} Q_{n}^{(i)}(j) \mathbb{1}_{\left[U_{j}, 1\right]}}_{=\hat{J}_{n}^{\leqslant k(i)}}-\underbrace{\frac{1}{n} \mathbb{1}\left\{\tilde{N}_{n}^{(i)}<k\right\} \sum_{j=\tilde{N}_{n}^{(i)}+1}^{k} Q_{n}^{(i)}(j) \mathbb{1}_{\left[U_{j}^{(i)}, 1\right]}}_{=\tilde{J}_{n}^{\leqslant k(i)}} .
$$

Note that this distributional equality holds jointly w.r.t. $i=1, \ldots, d$ due to the assumed independence. Let $F$ be a closed set and write

$$
\begin{aligned}
& \mathbf{P}\left(\left(\bar{J}_{n}^{k(1)}, \ldots, \bar{J}_{n}^{k(d)}\right) \in F\right) \\
& \leq \mathbf{P}\left(\left(\hat{J}_{n}^{\leqslant k(1)}, \ldots, \hat{J}_{n}^{\leqslant k(d)}\right) \in F, \sum_{i=1}^{d} \mathbb{1}\left\{\tilde{N}_{n}^{(i)}<k\right\}=0\right)+\sum_{i=1}^{d} \mathbf{P}\left(\mathbb{1}\left\{N_{n}^{(i)}<k\right\} \neq 0\right) \\
& \leq \mathbf{P}\left(\left(\hat{J}_{n}^{\leqslant k(1)}, \ldots, \hat{J}_{n}^{\leqslant k(d)}\right) \in F\right)+\sum_{i=1}^{d} \mathbf{P}\left(\mathbb{1}\left\{N_{n}^{(i)}<k\right\} \neq 0\right) .
\end{aligned}
$$


From Lemma 5.6 and the principle of the largest term,

$$
\begin{aligned}
& \limsup _{n \rightarrow \infty} \frac{\log \mathbf{P}\left(\left(\bar{J}_{n}^{k(1)}, \ldots, \bar{J}_{n}^{k(d)}\right) \in F\right)}{L(n) n^{\alpha}} \\
& \leq \limsup _{n \rightarrow \infty} \frac{\log \mathbf{P}\left(\left(\hat{J}_{n}^{\leq k(1)}, \ldots, \hat{J}_{n}^{\leq k(d)}\right) \in F\right)}{L(n) n^{\alpha}} \vee \max _{i=1, \ldots, d} \limsup _{n \rightarrow \infty} \frac{\log \mathbf{P}\left(\tilde{N}_{n}^{(i)}<k\right)}{L(n) n^{\alpha}} \\
& \leq-\inf _{\left(\xi_{1}, \ldots, \xi_{d}\right) \in F} I_{k}^{d}\left(\xi_{1}, \ldots, \xi_{d}\right) .
\end{aligned}
$$

Turning to the lower bound, let $G$ be an open set. Since the lower bound is trivial in case $\inf _{x \in G} I_{k}(x)=\infty$, we focus on the case $\inf _{x \in G} I_{k}(x)<\infty$. In this case, using a reasoning similar to the one leading to $(5.22)$,

$$
\begin{aligned}
& \liminf _{n \rightarrow \infty} \frac{\log \mathbf{P}\left(\left(\bar{J}_{n}^{k(1)}, \ldots, \bar{J}_{n}^{k(d)}\right) \in G\right)}{L(n) n^{\alpha}} \\
& \geq \liminf _{n \rightarrow \infty} \frac{\log \mathbf{P}\left(\left(\bar{J}_{n}^{k(1)}, \ldots, \bar{J}_{n}^{k(d)}\right) \in G, \sum_{i=1}^{d} \mathbb{1}\left\{\tilde{N}_{n}^{(i)} \geq k\right\}=0\right)}{L(n) n^{\alpha}} \\
& =\liminf _{n \rightarrow \infty} \frac{\log \mathbf{P}\left(\left(\hat{J}_{n}^{\leqslant k(1)}, \ldots, \hat{J}_{n}^{\leqslant k(d)}\right) \in G, \sum_{i=1}^{d} \mathbb{1}\left\{\tilde{N}_{n}^{(i)} \geq k\right\}=0\right)}{L(n) n^{\alpha}} \\
& \geq \liminf _{n \rightarrow \infty} \frac{1}{L(n) n^{\alpha}} \log \left(\mathbf{P}\left(\left(\hat{J}_{n}^{\leqslant k(1)}, \ldots, \hat{J}_{n}^{\leqslant k(d)}\right) \in G\right)-d \mathbf{P}\left(\tilde{N}_{n}^{(1)}<k\right)\right) \\
& =\liminf _{n \rightarrow \infty} \frac{1}{L(n) n^{\alpha}} \log \mathbf{P}\left(\left(\hat{J}_{n}^{\leqslant k(1)}, \ldots, \hat{J}_{n}^{\leqslant k(d)}\right) \in G\right) \\
& \geq-\inf _{\left(\xi_{1}, \ldots, \xi_{d}\right) \in G} I_{k}^{d}\left(\xi_{1}, \ldots, \xi_{d}\right) .
\end{aligned}
$$

The proof of Lemma 2.8 is completely analogous to the one-dimensional case, and therefore omitted.

\section{APPENDIX A: $M_{1}^{\prime}$ TOPOLOGY AND GOODNESS OF THE RATE FUNCTION}

Let $\tilde{\mathbb{D}}[0,1]$ be the space of functions from $[0,1]$ to $\mathbb{R}$ such that the left limit exists at each $t \in(0,1]$, the right limit exists at each $t \in[0,1)$, and

$$
\xi(t) \in[\xi(t-) \wedge \xi(t+), \xi(t-) \vee \xi(t+)]
$$

for each $t \in[0,1]$ where we interpret $\xi(0-)$ as 0 and $\xi(1+)$ as $\xi(1)$.

Definition 1. For $\xi \in \tilde{\mathbb{D}}$, define the extended completed graph $\Gamma^{\prime}(\xi)$ of $\xi$ as

$$
\Gamma^{\prime}(\xi) \triangleq\{(u, t) \in \mathbb{R} \times[0,1]: u \in[\xi(t-) \wedge \xi(t+), \xi(t-) \vee \xi(t+)]\},
$$

where $\xi(0-) \triangleq 0$ and $\xi(1+) \triangleq \xi(1)$. Define an order on the graph $\Gamma^{\prime}(\xi)$ by setting $\left(u_{1}, t_{1}\right)<\left(u_{2}, t_{2}\right)$ if either 
- $t_{1}<t_{2} ;$ or

- $t_{1}=t_{2}$ and $\left|\xi\left(t_{1}-\right)-u_{1}\right|<\left|\xi\left(t_{2}-\right)-u_{2}\right|$.

We call a continuous nondecreasing function $(u, t)=((u(s), t(s)), s \in[0,1])$ from $[0,1]$ to $\mathbb{R} \times[0,1]$ a parametrization of $\Gamma^{\prime}(\xi)$-or a parametrization of $\xi$-if $\Gamma^{\prime}(\xi)=\{(u(s), t(s)): s \in[0,1]\}$.

Definition 2. Define the $M_{1}^{\prime}$ metric on $\mathbb{D}$ as follows

$$
d_{M_{1}^{\prime}}(\xi, \zeta) \triangleq \inf _{\substack{(u, t) \in \Gamma^{\prime}(\xi) \\(v, r) \in \Gamma^{\prime}(\zeta)}}\left\{\|u-v\|_{\infty}+\|t-r\|_{\infty}\right\}
$$

Let $\mathbb{D}^{\uparrow} \triangleq\{\xi \in \mathbb{D}: \quad \xi$ is nondecreasing and $\xi(0) \geq 0\}$.

Proposition A.1. Suppose that $\hat{\xi}_{0} \in \tilde{\mathbb{D}}$ with $\hat{\xi}_{0}(0) \geq 0$ and $\xi_{n} \in \mathbb{D}^{\uparrow}$ for each $n \geq 1$. If $T \triangleq\{t \in$ $\left.[0,1]: \xi_{n}(t) \rightarrow \hat{\xi}_{0}(t)\right\}$ is dense on $[0,1]$ and $1 \in T$, then $\xi_{n} \stackrel{M_{1}^{\prime}}{\rightarrow} \xi_{0} \in \mathbb{D}^{\uparrow}$ where $\xi_{0}(t) \triangleq \lim _{s \downarrow t} \hat{\xi}_{0}(s)$ for $t \in[0,1)$ and $\xi_{0}(1) \triangleq \hat{\xi}_{0}(1)$.

Proof. It is easy to check that $\hat{\xi}_{0}$ has to be non-negative and non-decreasing, and for such $\hat{\xi}_{0}$, $\xi_{0}$ should be in $\mathbb{D}^{\uparrow}$. Let $(x, t)$ be a parametrization of $\Gamma^{\prime}\left(\hat{\xi}_{0}\right)$, and let $\epsilon>0$ be given. Note that $\Gamma^{\prime}\left(\xi_{0}\right)$ and $\Gamma^{\prime}\left(\hat{\xi}_{0}\right)$ coincide. Therefore, the proposition is proved if we show that there exists an integer $N_{0}$ such that for each $n \geq N_{0}, \Gamma^{\prime}\left(\xi_{n}\right)$ can be parametrized by some $(y, r)$ such that

$$
\|x-y\|_{\infty}+\|t-r\|_{\infty} \leq \epsilon
$$

We start with making an observation that one can always construct a finite number of points $S=\left\{s_{i}\right\}_{i=0,1, \ldots, m} \subseteq[0,1]$ such that

(S1) $0=s_{0}<s_{1}<\cdots<s_{m}=1$;

(S2) $t\left(s_{i}\right)-t\left(s_{i-1}\right)<\epsilon / 4$ for $i=1, \ldots, m$;

(S3) $x\left(s_{i}\right)-x\left(s_{i-1}\right)<\epsilon / 8$ for $i=1, \ldots, m$;

(S4) if $t\left(s_{k-1}\right)<t\left(s_{k}\right)<t\left(s_{k+1}\right)$ then $t\left(s_{k}\right) \in T$;

(S5) if $t\left(s_{k-1}\right)<t\left(s_{k}\right)=t\left(s_{k+1}\right)$, then $t\left(s_{k-1}\right) \in T$; if, in addition, $k-1>0$, then $t\left(s_{k-2}\right)<$ $t\left(s_{k-1}\right)$;

(S6) if $t\left(s_{k-1}\right)=t\left(s_{k}\right)<t\left(s_{k+1}\right)$, then $t\left(s_{k+1}\right) \in T$; if, in addition, $k+1<m$, then $t\left(s_{k+1}\right)<$ $t\left(s_{k+2}\right)$.

One way to construct such a set is to start with $S$ such that (S1), (S2), and (S3) are satisfied. This is always possible because $x$ and $t$ are continuous and non-decreasing. Suppose that (S4) is violated for some three consecutive points in $S$, say $s_{k-1}, s_{k}, s_{k+1}$. We argue that it is always possible to eliminate this violation by either adding an additional point $\hat{s}_{k}$ or moving $s_{k}$ slightly. More specifically, if there exists $\hat{s}_{k} \in\left(s_{k-1}, s_{k+1}\right) \backslash\left\{s_{k}\right\}$ such that $t\left(\hat{s}_{k}\right)=t\left(s_{k}\right)$, add $\hat{s}_{k}$ to $S$. If there is no such $\hat{s}_{k}, t(\cdot)$ has to be strictly increasing at $s_{k}$, and hence, from the continuity of $x$ and $t$ along with the fact that $T$ is dense, we can deduce that there has to be $\tilde{s}_{k} \in\left(s_{k-1}, s_{k+1}\right)$ such that $t\left(\tilde{s}_{k}\right) \in T$ and $\left|t\left(\tilde{s}_{k}\right)-t\left(s_{k}\right)\right|$ and $\left|x\left(\tilde{s}_{k}\right)-x\left(s_{k}\right)\right|$ are small enough so that (S2) and (S3) are still satisfied when we replace $s_{k}$ with $\tilde{s}_{k}$ in $S$. Iterating this procedure, we can construct $S$ so 
that (S1)-(S4) are satisfied. Now turning to (S5), suppose that it is violated for three consecutive points $s_{k-1}, s_{k}, s_{k+1}$ in $S$. Since $T$ is dense and $t$ is continuous, one can find $\hat{s}_{k}$ between $s_{k-1}$ and $s_{k}$ such that $t\left(s_{k-1}\right)<t\left(\hat{s}_{k}\right)<t\left(s_{k}\right)$ and $t\left(\hat{s}_{k}\right) \in T$. Note that after adding $\hat{s}_{k}$ to $S$, (S2), (S3), and (S4) should still hold while the number of triplets that violate (S5) is reduced by one. Repeating this procedure for each triplet that violates (S5), one can construct a new $S$ which satisfies (S1)(S5). One can also check that the same procedure for the triplets that violate (S6) can reduce the number of triplets that violate (S6) while not introducing any new violation for (S2), (S3), (S4), and (S5). Therefore, $S$ can be augmented so that the resulting finite set satisfies (S6) as well. Set $\hat{S} \triangleq\left\{s_{i} \in S: t\left(s_{i}\right) \in T, t\left(s_{i-1}\right)<t\left(s_{i}\right)\right.$ in case $i>0, t\left(s_{i}\right)<t\left(s_{i+1}\right)$ in case $\left.i<m\right\}$ and let $N_{0}$ be such that $n \geq N_{0}$ implies $\left|\xi_{n}\left(t\left(s_{i}\right)\right)-\hat{\xi}_{0}\left(t\left(s_{i}\right)\right)\right|<\epsilon / 8$ for all $s_{i} \in \hat{S}$. Now we will fix $n \geq N_{0}$ and proceed to showing that we can re-parametrize an arbitrary parametrization $\left(y^{\prime}, r^{\prime}\right)$ of $\Gamma\left(\xi_{n}\right)$ to obtain a new parametrization $(y, r)$ such that (A.2) is satisfied. Let $\left(y^{\prime}, r^{\prime}\right)$ be an arbitrary parametrization of $\Gamma\left(\xi_{n}\right)$. For each $i$ such that $s_{i} \in \hat{S}$, let $s_{i}^{\prime} \triangleq \max \left\{s \geq 0: r^{\prime}(s)=t\left(s_{i}\right)\right\}$ so that $r^{\prime}\left(s_{i}^{\prime}\right)=t\left(s_{i}\right)$ and $\xi_{n}\left(r^{\prime}\left(s_{i}^{\prime}\right)\right)=y^{\prime}\left(s_{i}^{\prime}\right)$. For $i$ 's such that $s_{i} \in S \backslash \hat{S}$, note that there are three possible cases: $t\left(s_{i}\right) \in(0,1), t\left(s_{i}\right)=0$, and $t\left(s_{i}\right)=1$. Since the other cases can be handled in similar (but simpler) manners, we focus on the case $t\left(s_{i}\right) \in(0,1)$. In this case, one can check that there exist $k$ and $j$ such that $k \leq i \leq k+j, t\left(s_{k-1}\right)<t\left(s_{k}\right)=t\left(s_{k+j}\right)<t\left(s_{k+j+1}\right)$, and $s_{k-1}, s_{k+j+1} \in \hat{S}$. Here we assume that $k>1$; the case $k=1$ is essentially identical but simpler-hence omitted. Note that from the monotonicity of $\hat{\xi}_{0}$ and (A.1),

$$
x\left(s_{k-2}\right) \leq \hat{\xi}_{0}\left(t\left(s_{k-2}\right)+\right) \leq \hat{\xi}_{0}\left(t\left(s_{k-1}\right)-\right) \leq \hat{\xi}_{0}\left(t\left(s_{k-1}\right)\right) \leq \hat{\xi}_{0}\left(t\left(s_{k-1}\right)+\right) \leq \hat{\xi}_{0}\left(t\left(s_{k}\right)-\right) \leq x\left(s_{k}\right),
$$

i.e., $\hat{\xi}_{0}\left(t\left(s_{k-1}\right)\right) \in\left[x\left(s_{k-2}\right), x\left(s_{k}\right)\right]$, which along with (S3) implies $\left|\hat{\xi}_{0}\left(t\left(s_{k-1}\right)\right)-x\left(s_{k-1}\right)\right|<\epsilon / 8$. From this, (S5), and the constructions of $s_{k-1}^{\prime}$ and $N_{0}$,

$$
\begin{aligned}
\left|y^{\prime}\left(s_{k-1}^{\prime}\right)-x\left(s_{k-1}\right)\right| & =\left|\xi_{n}\left(r^{\prime}\left(s_{k-1}^{\prime}\right)\right)-x\left(s_{k-1}\right)\right| \\
& =\left|\xi_{n}\left(r^{\prime}\left(s_{k-1}^{\prime}\right)\right)-\hat{\xi}_{0}\left(t\left(s_{k-1}\right)\right)\right|+\left|\hat{\xi}_{0}\left(t\left(s_{k-1}\right)\right)-x\left(s_{k-1}\right)\right| \\
& =\left|\xi_{n}\left(t\left(s_{k-1}\right)\right)-\hat{\xi}_{0}\left(t\left(s_{k-1}\right)\right)\right|+\left|\hat{\xi}_{0}\left(t\left(s_{k-1}\right)\right)-x\left(s_{k-1}\right)\right|<\epsilon / 4 .
\end{aligned}
$$

Following the same line of reasoning, we can show that $\left|y^{\prime}\left(s_{k+j+1}^{\prime}\right)-x\left(s_{k+j+1}\right)\right|<\epsilon / 4$. Noting that both $x$ and $y^{\prime}$ are nondecreasing, there have to exist $s_{k}^{\prime}, s_{k+1}^{\prime}, \ldots, s_{k+j}^{\prime}$ such that $s_{k-1}^{\prime}<s_{k}^{\prime}<$ $\cdots<s_{k+j}^{\prime}<s_{k+j+1}^{\prime}$ and $\left|y^{\prime}\left(s_{l}^{\prime}\right)-x\left(s_{l}\right)\right|<\epsilon / 4$ for $l=k, k+1, \ldots, k+j$. Note also that from (S2),

$$
\begin{aligned}
t\left(s_{l}\right)-\epsilon / 4 & =t\left(s_{k}\right)-\epsilon / 4<t\left(s_{k-1}\right)=r^{\prime}\left(s_{k-1}^{\prime}\right) \leq r^{\prime}\left(s_{l}^{\prime}\right) \leq r^{\prime}\left(s_{k+j+1}^{\prime}\right)=t\left(s_{k+j+1}\right)<t\left(s_{k+j}\right)+\epsilon / 4 \\
& =t\left(s_{l}\right)+\epsilon / 4,
\end{aligned}
$$

and hence, $\left|r^{\prime}\left(s_{l}^{\prime}\right)-t\left(s_{l}\right)\right|<\epsilon / 4$ for $l=k, \ldots, k+j$ as well. Repeating this procedure for the $i$ 's for which $s_{i}^{\prime}$ is not designated until there is no such $i$ 's are left, we can construct $s_{1}^{\prime}, \ldots, s_{m}^{\prime}$ in such a way that

$$
\left|y^{\prime}\left(s_{i}^{\prime}\right)-x\left(s_{i}\right)\right|<\epsilon / 4 \quad \text { and } \quad\left|r^{\prime}\left(s_{i}^{\prime}\right)-t\left(s_{i}\right)\right|<\epsilon / 4
$$

for all $i$ 's. Now, define a (piecewise linear) map $\lambda:[0,1] \rightarrow[0,1]$ by setting $\lambda\left(s_{i}\right)=s_{i}^{\prime}$ at each $s_{i}$ 's and interpolating $\left(s_{i}, s_{i}^{\prime}\right)$ 's in between. Then, $y \triangleq y^{\prime} \circ \lambda$ and $r \triangleq r^{\prime} \circ \lambda$ consist a parametrization 
$(y, r)$ of $\Gamma\left(\xi_{n}\right)$ such that $\left|x\left(s_{i}\right)-y\left(s_{i}\right)\right|<\epsilon / 4$ and $\left|t\left(s_{i}\right)-r\left(s_{i}\right)\right|<\epsilon / 4$ for each $i=1, \ldots, m$. Due to the monotonicity of $x, y, t$, and $r$ along with (S2) and (S3), we conclude that $\|y-x\|_{\infty}<\epsilon / 2$ and $\|t-r\|_{\infty}<\epsilon / 2$, proving (A.2).

Proposition A.2. Let $K$ be a subset of $\mathbb{D}^{\uparrow}$. If $M \triangleq \sup _{\xi \in K}\|\xi\|_{\infty}<\infty$ then $K$ is relatively compact w.r.t. the $M_{1}^{\prime}$ topology.

Proof. Let $\left\{\xi_{n}\right\}_{n=1,2, \ldots}$ be a sequence in $K$. We prove that there exists a subsequence $\left\{\xi_{n_{k}}\right\}_{k=1,2, \ldots}$ and $\xi_{0} \in \mathbb{D}$ such that $\xi_{n_{k}} \stackrel{M_{1}^{\prime}}{\rightarrow} \xi_{0}$ as $k \rightarrow \infty$. Let $T \triangleq\left\{t_{n}\right\}_{n=1,2, \ldots}$ be a dense subset of $[0,1]$ such that $1 \in T$. By the assumption, $\sup _{n=1,2, \ldots}\left|\xi_{n}\left(t_{1}\right)\right|<M$, and hence there is a subsequence $\left\{n_{k}^{(1)}\right\}_{k=1,2, \ldots}$ of $\{1,2, \ldots\}$ such that $\xi_{n_{k}^{(1)}}\left(t_{1}\right)$ converges to a real number $x_{1} \in[-M, M]$. For each $i \geq 1$, given $\left\{n_{k}^{(i)}\right\}$, one can find a further subsequence $\left\{n_{k}^{(i+1)}\right\}_{k=1,2, \ldots}$ of $\left\{n_{k}^{(i)}\right\}_{k=1,2, \ldots}$ in such a way that $\xi_{n_{k}^{(i+1)}}\left(t_{i+1}\right)$ converges to a real number $x_{i+1}$. Let $n_{k} \triangleq n_{k}^{(k)}$ for each $k=1,2, \ldots$ Then, $\xi_{n_{k}}\left(t_{i}\right) \rightarrow x_{i}$ as $k \rightarrow \infty$ for each $i=1,2, \ldots$ Define a function $\hat{\xi}_{0}: T \rightarrow \mathbb{R}$ on $T$ so that $\hat{\xi}_{0}\left(t_{i}\right)=x_{i}$. We claim that $\hat{\xi}_{0}$ has left limit everywhere; more precisely, we claim that for each $s \in(0,1]$, if a sequence $\left\{s_{n}\right\} \subseteq T \cap[0, s)$ is such that $s_{n} \rightarrow s$ as $n \rightarrow \infty$, then $\hat{\xi}_{0}\left(s_{n}\right)$ converges as $n \rightarrow \infty$. (With a similar argument, one can show that $\hat{\xi}_{0}$ has right limit everywhere-i.e., for each $s \in[0,1)$, if a sequence $\left\{s_{n}\right\} \subseteq T \cap(s, 1]$ is such that $s_{n} \rightarrow s$ as $n \rightarrow \infty$, then $\hat{\xi}_{0}\left(s_{n}\right)$ converges as $n \rightarrow \infty$.) To prove this claim, we proceed with proof by contradiction; suppose that the conclusion of the claim is not true -i.e., $\hat{\xi}_{0}\left(s_{n}\right)$ is not convergent. Then, there exist a $\epsilon>0$ and a subsequence $r_{n}$ of $s_{n}$ such that

$$
\left|\hat{\xi}_{0}\left(r_{n+1}\right)-\hat{\xi}_{0}\left(r_{n}\right)\right|>\epsilon
$$

Note that since $\hat{\xi}_{0}$ is a pointwise limit of nondecreasing functions $\left\{\xi_{n_{k}}\right\}$ (restricted on $T$ ),

- $\hat{\xi}_{0}$ is also nondecreasing on $T$,

- $\sup _{t \in T}\left|\hat{\xi}_{0}(t)\right|<M$.

(monotonicity)

(boundedness)

However, these two are contradictory to each other since the monotonicity together with (A.3) implies $\hat{\xi}_{0}\left(r_{N_{0}+j}\right)>\hat{\xi}_{0}\left(r_{N_{0}}\right)+j \epsilon$, which leads to the contradiction to the boundedness for a large enough $j$. This proves the claim.

Note that the above claim means that $\hat{\xi}_{0}$ has both left and right limit at each point of $T \cap(0,1)$, and due to the monotonicity, the function value has to be between the left limit and the right limit. Since $T$ is dense in $[0,1]$, we can extend $\hat{\xi}$ from $T$ to $[0,1]$ by setting $\hat{\xi}_{0}(t) \triangleq \lim _{\substack{t_{i} \rightarrow t \\ t_{i}>t}} \hat{\xi}_{0}\left(t_{i}\right)$ for $t \in[0,1] \backslash T$. Note that such $\hat{\xi}_{0}$ is an element of $\tilde{\mathbb{D}}$ and satisfies the conditions of Proposition A.1. We therefore conclude that $\xi_{n_{k}} \rightarrow \xi_{0} \in \mathbb{D}^{\uparrow}$ in $M_{1}^{\prime}$ as $k \rightarrow \infty$, where $\xi_{0}(t) \triangleq \lim _{s \downarrow t} \hat{\xi}_{0}(s)$ for $t \in[0,1)$ and $\xi_{0}(1) \triangleq \hat{\xi}_{0}(1)$. This proves that $K$ is indeed relatively compact.

Recall that our rate function for one-sided compound Poisson processes is as follows:

$$
I_{M_{1}^{\prime}}(\xi)= \begin{cases}\sum_{t \in[0,1]}(\xi(t)-\xi(t-))^{\alpha} & \text { if } \xi \text { is a non-decreasing pure jump function with } \xi(0) \geq 0 \\ \infty & \text { otherwise. }\end{cases}
$$


Proposition A.3. $I_{M_{1}^{\prime}}$ is a good rate function w.r.t. the $M_{1}^{\prime}$ topology.

Proof. In view of Proposition A.2, it is enough to show that the sublevel sets of $I_{M_{1}^{\prime}}$ are closed. Let $a$ be an arbitrary finite constant, and consider the sublevel set $\Psi_{I_{M_{1}^{\prime}}}(a) \triangleq\left\{\xi \in \mathbb{D}: I_{M_{1}^{\prime}}(\xi) \leq a\right\}$. Let $\xi^{c} \in \mathbb{D}$ be any given path that does not belong to $\Psi_{I_{M_{1}^{\prime}}}(a)$. We will show that there exists $\epsilon>0$ such that $d_{M_{1}^{\prime}}\left(\xi^{c}, \Psi_{I_{M_{1}^{\prime}}}(a)\right) \geq \epsilon$. Note that $\Psi_{I_{M_{1}^{\prime}}}(a)^{c}=A \cup B \cup C \cup D$ where

$A=\{\xi \in \mathbb{D}: \xi(0)<0\}$,

$B=\{\xi \in \mathbb{D}: \xi$ is not a non-decreasing function $\}$,

$C=\{\xi \in \mathbb{D}: \xi$ is non-decreasing but not a pure jump function $\}$,

$D=\left\{\xi \in \mathbb{D}: \xi\right.$ is a non-decreasing pure jump function with $\xi(0) \geq 0$ and $\left.\sum_{t \in[0,1]}(\xi(t)-\xi(t-))^{\alpha}>a\right\}$.

In each case, we will show that $\xi^{c}$ is bounded away from $\Psi_{I_{M_{1}^{\prime}}}(a)$. In case $\xi^{c} \in A$, note that for any parametrization $(x, t)$ of $\xi^{c}$, there has to be $s^{*} \in[0,1]$ such that $x\left(s^{*}\right)=\xi^{c}(0)<0$. On the other hand, $y(s) \geq 0$ for all $s \in[0,1]$ for any parametrization $(y, r)$ of $\zeta$ such that $\zeta \in \Psi_{I}(a)$, and hence, $\|x-y\|_{\infty} \geq \xi^{c}(0)$. Therefore,

$$
d_{M_{1}^{\prime}}\left(\xi^{c}, \zeta\right) \geq \inf _{\substack{(x, t) \in \Gamma\left(\xi^{c}\right) \\(y, r) \in \Gamma(\zeta)}}\|x-y\|_{\infty} \geq\left|\xi^{c}(0)\right|
$$

Since $\zeta$ was an arbitrary element of $\Psi_{I_{M_{1}^{\prime}}}(a)$, we conclude that $d_{M_{1}^{\prime}}\left(\xi^{c}, \Psi_{I}(a)\right) \geq \epsilon$ with $\epsilon=\left|\xi^{c}(0)\right|$.

Using a similar argument, it is straightforward to show that any $\xi^{c} \in B$ is bounded away from $\Psi_{I}(a)^{c}$.

If $\xi^{c} \in C$, there has to be $T_{s}$ and $T_{t}$ such that $0 \leq T_{s}<T_{t} \leq 1, \xi^{c}$ is continuous on $\left[T_{s}, T_{t}\right]$, and $c \triangleq \xi^{c}\left(T_{t}\right)-\xi^{c}\left(T_{s}\right)>0$. Pick a small enough $\epsilon \in(0,1)$ so that

$$
(4 \epsilon)^{\alpha-1}(c-5 \epsilon)>a .
$$

Note that since $\xi^{c}$ is uniformly continuous on $\left[T_{s}, T_{t}\right]$, there exists $\delta>0$ such that $\left|\xi^{c}(t)-\xi^{c}(s)\right|<\epsilon$ if $|t-s| \leq \delta$. In particular, we pick $\delta$ so that $\delta<\epsilon$ and $T_{s}+\delta<T_{t}-\delta$. We claim that

$$
d_{M_{1}^{\prime}}\left(\Psi_{I_{M_{1}^{\prime}}}(a), \xi^{c}\right) \geq \delta
$$

Suppose not. That is, there exists $\zeta \in \Psi_{I_{M_{1}^{\prime}}}(a)$ such that $d_{M_{1}^{\prime}}\left(\zeta, \xi^{c}\right)<\delta$. Let $(x, t) \in \Gamma\left(\xi^{c}\right)$ and $(y, r) \in \Gamma(\zeta)$ be the parametrizations of $\xi^{c}$ and $\zeta$, respectively, such that $\|x-y\|_{\infty}+\|t-r\|_{\infty}<\delta$. Since $I_{M_{1}^{\prime}}(\zeta) \leq a<\infty$, one can find a finite set $K \subseteq\{t \in[0,1]: \zeta(t)-\zeta(t-)>0\}$ of jump times of $\zeta$ in such a way that $\sum_{t \notin K}(\zeta(t)-\zeta(t-))^{\alpha}<\epsilon$. Note that since $\epsilon \in(0,1)$, this implies that $\sum_{t \notin K}(\zeta(t)-\zeta(t-))<\epsilon$. Let $T_{1}, \ldots, T_{k}$ denote (the totality of) the jump times of $\zeta$ in $K \cap\left(T_{s}+\delta, T_{t}-\delta\right]$, and let $T_{0} \triangleq T_{s}+\delta$ and $T_{k+1} \triangleq T_{t}-\delta$. That is, $\left\{T_{1}, \ldots, T_{k}\right\}=K \cap\left(T_{s}+\delta, T_{t}-\delta\right]=$ $K \cap\left(T_{0}, T_{k+1}\right]$. Note that 
- There exist $s_{0}$ and $s_{k+1}$ in $[0,1]$ such that

$$
y\left(s_{0}\right)=\zeta\left(T_{0}\right), \quad r\left(s_{0}\right)=T_{0}, \quad y\left(s_{k+1}\right)=\zeta\left(T_{k+1}\right), \quad r\left(s_{k+1}\right)=T_{k+1} .
$$

- For each $i=1, \ldots, k$, there exists $s_{i}^{+}$and $s_{i}^{-}$such that

$$
r\left(s_{i}^{+}\right)=r\left(s_{i}^{-}\right)=T_{i}, \quad y\left(s_{i}^{+}\right)=\zeta\left(T_{i}\right), \quad y\left(s_{i}^{-}\right)=\zeta\left(T_{i}-\right) .
$$

Since $t\left(s_{k+1}\right) \in\left[r\left(s_{k+1}\right)-\delta, r\left(s_{k+1}\right)+\delta\right] \subseteq\left[T_{s}, T_{t}\right]$, and $\xi^{c}$ is continuous on $\left[T_{s}, T_{t}\right]$ and nondecreasing,

$$
\begin{aligned}
y\left(s_{k+1}\right) & \geq x\left(s_{k+1}\right)-\delta=\xi^{c}\left(t\left(s_{k+1}\right)\right)-\delta \geq \xi^{c}\left(r\left(s_{k+1}\right)-\delta\right)-\delta=\xi^{c}\left(T_{k+1}-\delta\right)-\delta \\
& \geq \xi^{c}\left(T_{k+1}\right)-\epsilon-\delta \geq \xi^{c}\left(T_{k+1}\right)-2 \epsilon .
\end{aligned}
$$

Similarly,

$y\left(s_{0}\right) \leq x\left(s_{0}\right)+\delta=\xi^{c}\left(t\left(s_{0}\right)\right)+\delta \leq \xi^{c}\left(r\left(s_{0}\right)+\delta\right)+\delta=\xi^{c}\left(T_{0}+\delta\right)+\delta \leq \xi^{c}\left(T_{0}\right)+\epsilon+\delta \leq \xi^{c}\left(T_{0}\right)+2 \epsilon$.

Subtracting the two equations,

$$
y\left(s_{k+1}\right)-y\left(s_{0}\right) \geq \xi^{c}\left(T_{k+1}\right)-\xi^{c}\left(T_{0}\right)-4 \epsilon=c-4 \epsilon .
$$

Note that

$$
\begin{aligned}
\sum_{i=1}^{k}\left(\zeta\left(T_{i}\right)-\zeta\left(T_{i}-\right)\right) & =\zeta\left(T_{k+1}\right)-\zeta\left(T_{0}\right)-\sum_{t \in\left(T_{0}, T_{k+1}\right] \cap K^{c}}(\zeta(t)-\zeta(t-)) \geq \zeta\left(T_{k+1}\right)-\zeta\left(T_{0}\right)-\epsilon \\
& =y\left(s_{k+1}\right)-y\left(s_{0}\right)-\epsilon \geq c-5 \epsilon .
\end{aligned}
$$

On the other hand,

$$
\begin{aligned}
y\left(s_{i}^{+}\right)-y\left(s_{i}^{-}\right) & \leq\left(x\left(s_{i}^{+}\right)+\delta\right)-\left(x\left(s_{i}^{-}\right)-\delta\right)=x\left(s_{i}^{+}\right)-x\left(s_{i}^{-}\right)+2 \delta \leq \xi^{c}\left(t\left(s_{i}^{+}\right)\right)-\xi^{c}\left(t\left(s_{i}^{-}\right)\right)+2 \delta \\
& \leq \xi^{c}\left(r\left(s_{i}^{+}\right)+\delta\right)-\xi^{c}\left(r\left(s_{i}^{-}\right)-\delta\right)+2 \delta \leq \xi^{c}\left(T_{i}+\delta\right)-\xi^{c}\left(T_{i}-\delta\right)+2 \delta \leq 2 \epsilon+2 \delta \leq 4 \epsilon .
\end{aligned}
$$

That is, $\left(\zeta\left(T_{i}\right)-\zeta\left(T_{i}-\right)\right)^{\alpha-1}=\left(y\left(s_{i}^{+}\right)-y\left(s_{i}^{-}\right)\right)^{\alpha-1} \geq(4 \epsilon)^{\alpha-1}$. Combining this with (A.5),

$I_{M_{1}^{\prime}}(\zeta) \geq \sum_{i=1}^{k}\left(\zeta\left(T_{i}\right)-\zeta\left(T_{i}-\right)\right)^{\alpha}=\sum_{i=1}^{k}\left(\zeta\left(T_{i}\right)-\zeta\left(T_{i}-\right)\right)\left(\zeta\left(T_{i}\right)-\zeta\left(T_{i}-\right)\right)^{\alpha-1} \geq(c-5 \epsilon)(4 \epsilon)^{\alpha-1}>a$,

which is contradictory to the assumption that $\zeta \in \Psi_{I_{M_{1}^{\prime}}}(a)$. Therefore, the claim that $\xi^{c}$ is bounded away from $\Psi_{I_{M_{1}^{\prime}}}(a)$ by $\delta$ is proved.

Finally, suppose that $\xi^{c} \in D$. That is, there exists $\left\{\left(z_{i}, u_{i}\right) \in \mathbb{R}_{+} \times[0,1]\right\}_{i=1, \ldots}$ such that $\xi^{c}=\sum_{i=1}^{\infty} z_{i} \mathbb{1}_{\left[u_{i}, 1\right]}$ where $u_{i}$ 's are all distinct and $\sum_{i=1}^{\infty} z_{i}^{\alpha}>a$. Pick sufficiently large $k$ and sufficiently small $\delta>0$ such that $\sum_{i=1}^{k}\left(z_{i}-2 \delta\right)^{\alpha}>a$ and $u_{i+1}-u_{i}>2 \delta$ for $i=1, \ldots, k-1$. We claim that $d_{M_{1}^{\prime}}\left(\zeta, \xi^{c}\right) \geq \delta$ for any $\zeta \in \Psi_{I_{M_{1}^{\prime}}}(a)$. Suppose not and there is $\zeta \in \Psi_{I_{M_{1}^{\prime}}}(a)$ such that 
$\|x-y\|_{\infty}+\|t-r\|_{\infty}<\delta$ for some parametrizations $(x, t) \in \Gamma\left(\xi^{c}\right)$ and $(y, r) \in \Gamma(\zeta)$. Note first that there are $s_{i}^{+}$'s and $s_{i}^{-}$'s for each $i=1, \ldots, k$ such that $t\left(s_{i}^{-}\right)=t\left(s_{i}^{+}\right)=u_{i}, x\left(s_{i}^{-}\right)=\xi^{c}\left(u_{i}-\right)$, and $x\left(s_{i}^{+}\right)=\xi^{c}\left(u_{i}\right)$. Since $y\left(s_{i}^{+}\right) \geq x\left(s_{i}^{+}\right)-\delta=\xi^{c}\left(u_{i}\right)-\delta$ and $y\left(s_{i}^{-}\right) \leq x\left(s_{i}^{-}\right)+\delta=\xi^{c}\left(u_{i}-\right)+\delta$,

$$
\zeta\left(r\left(s_{i}^{+}\right)\right)-\zeta\left(r\left(s_{i}^{-}\right)\right) \geq y\left(s_{i}^{+}\right)-y\left(s_{i}^{-}\right) \geq \xi^{c}\left(u_{i}\right)-\xi^{c}\left(u_{i}^{-}\right)-2 \delta=z_{i}-2 \delta .
$$

Note that by construction, $r\left(s_{i}^{+}\right)<t\left(s_{i}^{+}\right)+\delta=u_{i}+\delta<u_{i+1}-\delta=t\left(s_{i+1}^{-}\right)-\delta<r\left(s_{i+1}^{-}\right)$for each $i=1, \ldots, k-1$, and hence, along with the subadditivity of $x \mapsto x^{\alpha}$,

$$
I_{M_{1}^{\prime}}(\zeta)=\sum_{t \in[0,1]}(\zeta(t)-\zeta(t-))^{\alpha} \geq \sum_{i=1}^{k}\left[\zeta\left(r\left(s_{i}^{+}\right)\right)-\zeta\left(r\left(s_{i}^{-}\right)\right)\right]^{\alpha} \geq \sum_{i=1}^{k}\left(z_{i}-2 \delta\right)^{\alpha}>a,
$$

which is contradictory to the assumption $\zeta \in \Psi_{I_{M_{1}^{\prime}}}(a)$.

\section{REFERENCES}

Asmussen, S. and Pihlsgi̊n, M. (2005). Performance analysis with truncated heavy-tailed distributions. Methodol. Comput. Appl. Probab. 7 439-457. MR2235154

Bazhba, M., Blanchet, J., Rhee, C.-H. and Zwart, B. (2019). Queue length asymptotics for the multiple server queue with heavy-tailed Weibull service times. Queueing Systems.

Blanchet, J., Glynn, P. and Meyn, S. (2011). Large deviations for the empirical mean of an M/M/1 queue. Queueing Systems: Theory and Applications 73 425-446.

Borovkov, A. A. and Borovkov, K. A. (2008). Asymptotic analysis of random walks: Heavy-tailed distributions 118. Cambridge University Press.

Borovkov, A. A. and Mogulskit, A. A. (2010). On large deviation principles in metric spaces. Siberian Mathematical Journal 51 989-1003.

Dembo, A. and Zeitouni, O. (2009). Large deviations techniques and applications 38. Springer Science \& Business Media.

Dembo, A. and Zeitouni, O. (2010). Large deviations techniques and applications. Springer-Verlag, Berlin.

Denisov, D., Dieker, A. and Shneer, V. (2008). Large deviations for random walks under subexponentiality: the big-jump domain. The Annals of Probability 36 1946-1991.

Donsker, M. and VARAdhan, S. (1976). Asymptotic evaluation of certain Markov process expectations for large timeIII. Comm. in Pure and Applied Math. 29 389-461.

Duffy, K. R., Macci, C. and Torrisi, G. L. (2011). Sample path large deviations for order statistics. Journal of Applied Probability 48 238-257.

Duffy, K. R. and Meyn, S. P. (2014). Large deviation asymptotics for busy periods. Stoch. Syst. $4300-319$. MR3353220

Duffy, K. R. and SApozhnikov, A. (2008). The large deviation principle for the on-off Weibull sojourn process. J. Appl. Probab. 45 107-117. MR2409314

Embrechts, P., Klüppelberg, C. and Mikosch, T. (1997). Modelling extremal events. Applications of Mathematics (New York) 33. Springer-Verlag, Berlin For insurance and finance. MR1458613

Foss, S., Korshunov, D. and Zachary, S. (2011). An introduction to heavy-tailed and subexponential distributions. Springer.

Ganesh, A. J., O'Connell, N. and Wischik, D. J. (2004). Big queues. Springer.

Gantert, N. (1998). Functional Erdos-Renyi Laws for Semiexponential Random Variables. The Annals of Probability 26 1356-1369.

Gantert, N., Ramanan, K. and Rembart, F. (2014). Large deviations for weighted sums of stretched exponential random variables. Electron. Commun. Probab. 19 no. 41, 14. MR3233203

Jelenković, P. and Momčılović, P. (2003). Large deviation analysis of subexponential waiting times in a processor-sharing queue. Mathematics of Operations Research 28 587-608. 
Kontoyiannis, I. and Meyn, S. (2005). Large Deviations Asymptotics and the Spectral Theory of Multiplicatively Regular Markov Processes. Electron. J. Probab. 10 61-123.

Lindskog, F., Resnick, S. I. and Roy, J. (2014). Regularly varying measures on metric spaces: Hidden regular variation and hidden jumps. Probability Surveys 11 270-314.

Mogulskit, A. (1993). Large deviations for processes with independent increments. The Annals of Probability $202-215$

NagAev, A. V. (1969). Limit theorems that take into account large deviations when Cramér's condition is violated. Izv. Akad. Nauk UzSSR Ser. Fiz.-Mat. Nauk 13 17-22. MR0282396

NagAev, A. V. (1977). A property of sums of independent random variables. Teor. Verojatnost. $i$ Primenen. 22 335-346. MR0438438

Puhalskit, A. A. and Whitt, W. (1997). Functional large deviation principles for first-passage-time processes. The Annals of Applied Probability 362-381.

Resnick, S. I. (2007). Heavy-tail phenomena. Springer Series in Operations Research and Financial Engineering. Springer, New York Probabilistic and statistical modeling. MR2271424

Rhee, C.-H., Blanchet, J. and Zwart, B. (2016). Sample Path Large Deviations for Heavy-Tailed Lévy Processes and Random Walks. eprint arXiv:1606.02795.

Whiт,, W. (2000). The impact of a heavy-tailed service-time distribution upon the $M / G I / s$ waiting-time distribution. Queueing Systems Theory Appl. 36 71-87. MR1806961

Science Park 123, Amsterdam, The Netherlands

475 Via Ortega, Stanford, California, USA

E-MAIL: bazhba@cwi.nl; bert.zwart@cwi.nl

E-MAIL: jblanche@stanford.edu

2145 Sheridan Road, Evanston, Illinois, USA

E-MAIL: chang-han.rhee@northwestern.edu 